\title{
Applications of Flavor Symmetry to the Phenomenology of Elementary Particles*
}

\author{
(Ph.D. Thesis, University of California at Berkeley)
}

\author{
Thomas A. Kaeding ${ }^{\dagger}$ \\ Theoretical Physics Group \\ Lawrence Berkeley Laboratory \\ University of California \\ Berkeley, California 94720
}

\begin{abstract}
Some applications of flavor symmetry are examined. Approximate flavor symmetries and their consequences in the MSSM are considered, and found to give natural values for the possible $B$ - and $L$-violating couplings that are empirically acceptable, except for the case of proton decay. The coupling constants of $S U(3)$ are calculated and used to parameterize the decays of the $D$ mesons in broken flavor $S U(3)$. The resulting couplings are used to estimate the long-distance contributions to $D$-meson mixing.
\end{abstract}

*This work was supported by the Director, Office of Energy Research, Office of High Energy and Nuclear Physics, Division of High Energy Physics of the U.S. Department of Energy under Contract DE-AC03-76SF00098.

$\dagger$ Electronic address: kaedin@theorm.1bl.gov. 


\section{DISCLAIMER}

This report was prepared as an account of work sponsored by an agency of the United States Government. Neither the United States Government nor any agency thereof, nor any of their employees, make any warranty, express or implied, or assumes any legal liability or responsibility for the accuracy, completeness, or usefulness of any information, apparatus, product, or process disclosed, or represents that its use would not infringe privately owned rights. Reference herein to any specific commercial product, process, or service by trade name, trademark, manufacturer, or otherwise does not necessarily constitute or imply its endorsement, recommendation, or favoring by the United States Government or any agency thereof. The views and opinions of authors expressed herein do not necessarily state or reflect those of the United States Government or any agency thereof. 


\section{DISCLAIMER}

Portions of this document may be illegible in electronic image products. Images are produced from the best available original document. 


\title{
Applications of Flavor Symmetry to the Phenomenology of Elementary Particles
}

\author{
Copyright @ 1995
}

by

Thomas Allan Kaeding

The U.S. Department of Energy has the right to use this document for any purpose whatsoever including the right to reproduce all or any part thereof 
Applications of Flavor Symmetry to the Phenomenology of Elementary Particles

by

Thomas Allan Kaeding II

B.S. (University of Michigan) 1988

M.A. (University of California at Berkeley) 1990

A dissertation submitted in partial satisfaction of the requirements for the degree of

Doctor of Philosophy

in

Physics

in the

GRADUATE DIVISION

of the

UNIVERSITY of CALIFORNIA at BERKELEY

Committee in charge:

Professor Lawrence J. Hall, Co-Chair

Doctor Ian Hinchliffe, Co-Chair

Professor Mary K. Gaillard

Professor John O. Rasmussen 


\author{
Abstract \\ Applications of Flavor Symmetry to the \\ Phenomenology of Elementary Particles \\ by \\ Thomas Allan Kaeding II \\ Doctor of Philosophy in Physics \\ University of California at Berkeley \\ Professor Lawrence J. Hall, Co-Chair \\ Doctor Ian Hinchliffe, Co-Chair
}

This work is a collection of applications of flavor symmetries to the understanding of the interactions of elementary particles.

In Chapter 2 we will investigate the implications of approximate flavor symmetries on the couplings in the minimal supersymmetric standard model. We will find that the natural values of the couplings that violate baryon or lepton number under these symmetries are below the limits set by experimental searches. There is one exception to this pattern: the case of proton decay. In order to suppress the decay of the proton, we will consider discrete symmetries, and in particular generalized $R$-parities.

Chapter 3 deals with the calculation of coupling coefficients in the group of $S U(3)$. These coefficients are needed for the remainder of this work. Such calculations form an industry of their own and their results are useful in other areas of 
physics. Therefore, a discussion of the calculation is warranted.

One application of the coefficients of Chapter 3 is a study of meson decay in flavor $S U(3)$. We study the decays of the $D$ mesons to two-body hadronic states in broken $S U(3)$ in Chapter 4. We parameterize the modes completely. By applying a few simplifying assumptions, we can reduce the number of parameters such that they can be fit by the available data. This fit is performed, the expected large symmetry breaking is found, and predictions are made when possible for unmeasured modes.

The couplings of the $D$ mesons to the pseudoscalar and vector mesons found in Chapter 4 are applied to the problem of meson mixing in Chapter 5. Here we estimate the long-distance contributions to the mixing of the $D$ mesons and find that the large $S U(3)$ breaking gives the possibility of large mixing between $D^{0}$ and $\bar{D}^{0}$. 
To Natasha, my faithful companion for so many years 


\section{Contents}

List of Figures vii

List of Tables viii

Acknowledgements ix

$\begin{array}{ll}\text { Introduction } & 1\end{array}$

1 Fundamentals of Particle Physics 3

1.1 The Standard Model 3

1.1.1 Particle Multiplets and Gauge Interactions 4

1.1.2 The Higgs Mechanism and Particle Masses 6

$\begin{array}{lll}\text { 1.1.3 Fermion Mixing } & 9\end{array}$

$\begin{array}{ll}1.1 .4 & \text { Discrete Symmetries } \\ & 10\end{array}$

$\begin{array}{ll}\text { 1.1.5 Color Confinement } & 10\end{array}$

$\begin{array}{lll}1.2 & \text { Grand Unification } & 10\end{array}$

$\begin{array}{lll}1.3 & \text { Supersymmetry } & 12\end{array}$

2 Approximate and Discrete Symmetries in the MSSM 14

2.1 Couplings in the MSSM 14

2.2 Experimental Limits 16

2.2.1 Proton Decay 17

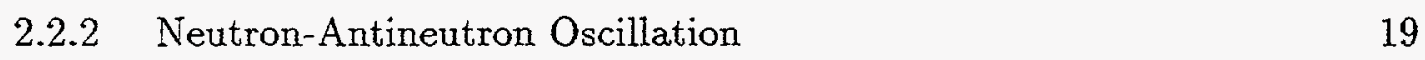

2.2.3 Exotic Lepton Decays 22

2.2.4 Neutrino Oscillation 24

2.3 Approximate Flavor Symmetries and Natural Values for Couplings 24

2.4 Discrete Symmetries and Proton Decay 26

2.5 Generalized $R$-Parities $\quad 30$

2.6 Conclusions 32

$3 \quad S U(3)$ Coupling Coefficients $\quad 33$

3.1 Basic Concepts 34

3.2 Irreducible Representations $\quad 37$ 
3.3 Tensor Decomposition $\quad 38$

3.4 Isoscalar Factors $\quad 40$

3.5 Computer Implementation 42

3.5.1 Tensors 44

3.5.2 Representations 45

3.5.3 The Table of Coefficients 45

3.5.4 The Tensor Decomposition 45

3.5.5 Isoscalar Factor Extraction 46

3.6 The Tables of Isoscalar Factors 46

$4 \quad D$ Meson Decays in Broken Flavor $S U(3)$

4.1 Particle States in Flavor $S U(3) \quad 60$

4.2 The Weak Hamiltonian 61

4.2.1 $H_{\text {weak }}$ in Terms of $S U(3)$ Representations 61

$\begin{array}{lll}4.2 .2 & \text { QCD Evolution } & 62\end{array}$

4.3 Parameterization 63

4.3.1 SU(3) Breaking $\quad 63$

4.3.2 Reduced Matrix Elements 63

4.3.3 Linear Combinations of Reduced Matrix Elements 66

4.4 Data and Fitting Thereof 68

$\begin{array}{lll}4.5 & \text { Predictions } & 75\end{array}$

4.5.1 Predictions from the Fit Parameters 75

4.5.2 Unconstrained Linear Combinations 75

4.5.3 Modes Involving Axial Vectors 80

$\begin{array}{lll}4.6 & \text { Comments on Models } & 80\end{array}$

4.7 Conclusions 83

5 . D-Meson Mixing in Broken Flavor $S U(3) \quad 87$

5.1 Short- and Long-Distance Contributions 88

5.2 Estimate in HQEFT 91

5.3 Mixing Estimate in Broken $S U(3) \quad 92$ 
5.4 Conclusions

References 


\section{List of Figures}

Figure 2.1 Contribution to proton decay involving $\lambda^{(2)}$ and $\lambda^{(3)}$. 18

$\begin{array}{lll}\text { Figure 2.2 Contribution to proton decay involving } \kappa^{(1)} \text {. } & 18\end{array}$

Figure 2.3 Contribution to proton decay involving $\kappa^{(2)}$. 19

Figure 2.4 Contribution to neutron oscillation involving $\lambda^{(3)}$. 20

Figure 2.5 Contribution to muon decay involving $\lambda^{(1)}$. 22

Figure 2.6 Contribution to muon decay involving $\kappa^{(6)}$. 23

Figure 2.7 Contribution to tau decay involving $\kappa^{(4)}$. 23

Figure 4.1 Number of events versus helicity angle in the analysis of $\quad 70$ the decay $D_{s} \rightarrow \eta^{\prime} \rho^{+}$.

Figure 4.2 External spectator diagram in the decays of $D$ mesons. 83

Figure 4.3 Internal spectator diagram in the decays of $D$ mesons. 84

Figure 4.4 External annihilation diagram in the decays of $D$ mesons. 84

Figure 4.5 Internal annihilation diagram in the decays of $D$ mesons. $\quad 85$

Figure 4.6 External exchange diagram in the decays of $D$ mesons. 85

Figure 4.7 Internal exchange diagram in the decays of $D$ mesons. 86

$\begin{array}{lll}\text { Figure } 5.1 \text { Box diagrams in } D \text {-meson mixing. } & 89\end{array}$

Figure 5.2 Two-body long-distance contriution to $D$-meson mixing. $\quad 91$ 


\section{List of Tables}

Table 1.1 Charge $c_{1}$ appearing in the covariant derivative. 6

Table $3.1 \quad$ List of tables of isoscalar factors. 48

Table $3.2 \quad$ Phase factors $\xi$. 49

Table 3.3 Phase factors $\zeta$. 49

Table $3.4 \quad$ Isoscalar factors for $3 \otimes 3$. 50

Table $3.5 \quad$ Isoscalar factors for $\overline{\mathbf{3}} \otimes \mathbf{3}$. 50

Table $3.6 \quad$ Isoscalar factors for $6 \otimes 3$. 50

Table $3.7 \quad$ Isoscalar factors for $6 \otimes \overline{\mathbf{3}}$. 51

Table $3.8 \quad$ Isoscalar factors for $8 \otimes 3$.

Table $3.9 \quad$ Isoscalar factors for $8 \otimes \mathbf{8}$.

Table $3.10 \quad$ Isoscalar factors for $10 \otimes 8$. 53

Table 3.11 Isoscalar factors for $\overline{\mathbf{1 0}} \otimes \mathbf{1 0}$. 54

Table 3.12 Isoscalar factors for $\mathbf{1 5} \otimes \overline{\mathbf{3}}$.

Table $3.13 \quad$ Isoscalar factors for $27 \otimes 8$. 56

Table 3.14 Isoscalar factors for $27 \otimes 27$ to the singlet only. 58

Table 4.1 List of reduced matrix elements. 65

Table 4.2 Linear combinations and their fit values. $\quad 71$

Table 4.3 The remaining parameters and their fit values. 72

Table 4.4 Modes with positive experimental values. 73

Table 4.5 $D^{0}$ modes with predicted branching ratios. $\quad 76$

Table 4.6 $\quad D^{+}$modes with predicted branching ratios. $\quad 77$

$\begin{array}{lll}\text { Table } 4.7 & D_{s} \text { modes with predicted branching ratios. } & 78\end{array}$

Table 4.8 Modes based on estimates. 81

Table 5.1 Estimates of $\Delta m_{D}^{\mathrm{L}-\mathrm{D}}$. $\quad 92$ 


\section{Acknowledgements}

My greatest gratitude goes to my advisor, Ian Hinchliffe. Without him this document would not have the form that it has, or perhaps no form at all.

A few additional people deserve credit for help with some details. Thanks go to M. Sher for pointing out errors in [1], which led to a revision of Section 2.2.2. Thanks go to J. J. de Swart for an explanation of his phase conventions in $S U(3)$. Thanks go also to G. H. Trilling for hints in reconciling the treatment of charm meson data with experimental limits to that with positive values.

Most of chapter 2 was reprinted with permission from Physical Review D vol. 47 (1993), pp. 279-284. Copyright 1993 The American Physical Society. The earliest parts of this work were supported by a National Science Foundation Graduate Fellowship. The remainder was supported by the Director, Office of Energy Research, Office of High Energy and Nuclear Physics, Division of High Energy Physics of the U.S. Department of Energy under Contract DE-AC03-76SF00098. This document was typeset with $\mathrm{IAT}_{\mathrm{E}} \mathrm{X}$ [2]. Today's thesis was brought to you by the letter $M$ and the number 3 . 


\section{Introduction}

Symmetry, in our humble opinion, is one of the most beautiful aspects of any science. More so when applied to the understanding of fundamental particles and forces. This work is a collection of topics in flavor symmetry in the physics of elementary particles.

In Chapter 2 we will investigate the implications of approximate flavor symmetries on the couplings in the minimal supersymmetric standard model. We will find that the natural values of the couplings that violate baryon or lepton number under these symmetries are below the limits set by experimental searches. There is one exception to this pattern: the case of proton decay. In order to suppress the decay of the proton, we will consider discrete symmetries, and in particular generalized $R$-parities.

Chapter 3 deals with the calculation of coupling coefficients in the group of $S U(3)$. These coefficients are needed for the remainder of this work. Such calculations form an industry of their own and their results are useful in other areas of physics. Therefore, a discussion of the calculation is warranted.

One application of the coefficients of Chapter 3 is a study of meson decay in flavor $S U(3)$. We study the decays of the $D$ mesons to two-body hadronic states in broken $S U(3)$ in Chapter 4. We parameterize the modes completely. By applying a few simplifying assumptions, we can reduce the number of parameters such that they can be fit by the available data. This fit is performed, the expected large symmetry breaking is found, and predictions are made when possible for unmeasured modes. 
The couplings of the $D$ mesons to the pseudoscalar and vector mesons found in Chapter 4 are applied to the problem of meson mixing in Chapter 5. Here we estimate the long-distance contributions to the mixing of the $D$ mesons and find that the large $S U(3)$ breaking gives the possibility of large mixing between $D^{0}$ and $\bar{D}^{0}$. 


\section{Fundamentals of Particle Physics}

This thesis builds upon ideas that have been in particle physics for many years. This chapter is intended as an introduction to them. The Standard Model (SM) has been very successful in describing the interactions of elementary particles, even in the light of the precision data of recent years [3]. Beyond the standard model, attempts have been made to unify the forces into one single force of nature. Such theories fall under the title of Grand Unification (GUT) [4]. However, it has recently been discovered that a viable GUT requires that nature also be supersymmetric at high energies [5]. Hence we have the need for Supersymmetry (SUSY), the symmetry relating particles of different spin. In this introductory chapter we will present briefly the standard model of particle physics and the ideas of grand unification and supersymmetry.

\subsection{The Standard Model}

Here we will cover the main points of the standard model of particle physics. Topics included are the gauge interactions, the Higgs mechanism, the discrete symmetries found in the model, fermion mixing, and color confinement. A good introductory volume with the top-down approach to the standard model is [6]. Readers interested in a more detailed exposition are directed to [7] [8]. 


\subsubsection{Particle Multiplets and Gauge Interactions}

The standard model is based upon the gauge interactions described by the group

$$
G_{\mathrm{SM}}=S U(3)_{\mathrm{color}} \times S U(2)_{\text {left }} \times U(1)_{Y}
$$

which breaks via the Higgs mechanism [9] at low energies to the group

$$
G_{\mathrm{SM}} \rightarrow S U(3)_{\text {color }} \times U(1)_{\mathrm{EM}}
$$

The fermions are assigned to singlets and fundamental representations of the irreducible subgroups of $G_{\mathrm{SM}}$. There are known (so far) to be three "generations" of fermions, differing only in mass, but identical in their interactions. The bosons responsible for mediating the fundamental forces are assigned to the adjoint representations. Until we discuss fermion mixing, we will limit our discussion to one generation of elementary particles.

The particles of the first generation (the electron and its neutrino, and the up and down quarks) are assigned to $S U(2)$ multiplets in the following way:

$$
\begin{aligned}
& e_{R}, \\
& l=\left(\begin{array}{l}
\nu \\
e
\end{array}\right)_{L}, \\
& u_{R}^{\alpha}, \\
& d_{R}^{\alpha}, \\
& q^{\alpha}=\left(\begin{array}{c}
u^{\alpha} \\
d^{\alpha}
\end{array}\right)_{L},
\end{aligned}
$$

where $R$ and $L$ subscripts differentiate right- and left-handed states and the index $\alpha$ is a color $S U(3)$ index (running over "red," "yellow," and "blue"). In order to build gauge-invariant interactions for these fermions, we need to introduce the covariant derivative

$$
\mathcal{D}^{\mu} \equiv \partial^{\mu}-i \frac{g_{1}}{2} c_{1} B^{\mu}-i \frac{g_{2}}{2} c_{2} \tau_{i} W_{i}^{\mu}-i \frac{g_{3}}{2} c_{3} \lambda_{a} G_{a}^{\mu} .
$$


Here the $g_{i}$ are the three coupling constants. The charge $c_{1}$ is the usual hypercharge; its values are listed in Table 1.1. The charge $c_{2}$ is 1 for the weak doublets $l, q$, and $h$ (introduced below), and is 0 otherwise. The charge $c_{3}$ is 1 for colored particles ( $u_{R}$, $\left.d_{R}, q\right)$, and 0 otherwise. The index $i$ runs over the three components of the adjoint representation of $S U(2)$, and $a$ over the eight components of the adjoint of $S U(3)$. The $\tau_{i}$ and $\lambda_{a}$ are the Pauli and Gell-Mann matrices acting on the fundamental representations of these groups. The $\tau_{i}$ and $\lambda_{a}$ carry two suppressed indices, one of which is contracted with the index on the fermion on which the covariant derivative acts; the other is contracted with the index on $\bar{f}$ in Equation 1.5 below. The new fields are the gauge fields $B^{\mu}, W_{i}^{\mu}$, and $G_{a}^{\mu}$, corresponding respectively to the $U(1)$, $S U(2)$, and $S U(3)$ of Equation 1.1.

We are now ready to write the gauge-invariant Lagrangian for the fermion fields. It is

$$
\mathcal{L}_{\text {fermions }}=i \bar{f} \gamma^{\mu} \mathcal{D}_{\mu} f
$$

where $f$ runs over the multiplets in Equation 1.3. We have adopted the convention that repeated indices are summed over. This applies also to the suppressed weak and color indices in Equation 1.5. When the covariant derivative of Equation 1.4 is inserted into the Lagrangian, the usual kinetic energy and gauge interaction terms are found.

What remains is to write a gauge-invariant Lagrangian which includes the kinetic energy and self-interactions of the gauge fields $B^{\mu}, W_{i}^{\mu}$, and $G_{a}^{\mu}$. For this purpose we will define for some gauge field $X_{i}^{\mu}$ a tensor

$$
X_{i}^{\mu \nu} \equiv \partial^{\nu} X_{i}^{\mu}-\partial^{\mu} X_{i}^{\nu}-i g\left[X^{\mu}, X^{\nu}\right]_{i}
$$

where $X^{\mu}$ without an index is the contraction on $X_{i}^{\mu}$ with the matrices of the adjoint representation of the appropriate group, and $[,]_{i}$ represents the $i$ th component of the commutator. For the $B^{\mu}$, the commutator vanishes, and so $B^{\mu \nu}$ has the same form as the electromagnetic field-strength tensor. The Lagrangian for the gauge 
Table 1.1: Charge $c_{1}$ appearing in the covariant derivative. The fields are those on which the covariant derivative acts.

\begin{tabular}{|c|c|}
\hline field & $c_{1}$ \\
\hline$e_{R}$ & -2 \\
$l$ & -1 \\
$u_{R}$ & $\frac{4}{3}$ \\
$d_{R}$ & $-\frac{2}{3}$ \\
$q$ & $\frac{1}{3}$ \\
$h$ & 1 \\
\hline
\end{tabular}

fields can now be written in a gauge-invariant form as

$$
\mathcal{L}_{\text {gauge }}=-\frac{1}{4} B_{\mu \nu} B^{\mu \nu}-\frac{1}{4} W_{i \mu \nu} W_{i}^{\mu \nu}-\frac{1}{4} G_{a \mu \nu} G_{a}^{\mu \nu}
$$

When the tensors of Equation 1.6 are inserted into this Lagrangian, what result are the kinetic energies and cubic and quartic self-interactions of the $W^{\mu}$ and $G^{\mu}$ fields.

\subsubsection{The Higgs Mechanism and Particle Masses}

There are two problems with the standard model described so far. First, the gauge symmetry of $G_{\mathrm{SM}}$ will not allow fermion-mass terms of the form

$$
m_{f} \bar{f} f=m_{f} \bar{f}_{R} f_{L}+m_{f} \bar{f}_{L} f_{R}
$$

or boson-mass terms of the form

$$
\frac{1}{2} M_{X}^{2} X^{\mu} X_{\mu}
$$

since these combinations are not singlets under the $S U(2)$ group. Second, the physics of low energies does not display the symmetry of the group in Equation 1.1. 
Instead, this group must be broken to the group in Equation 1.2. Both of these problems are solved by the Higgs mechanism.

We introduce a new $S U(2)$ doublet $h$

$$
h=\left(\begin{array}{l}
h^{+} \\
h^{0}
\end{array}\right)
$$

and its Lagrangian

$$
\mathcal{L}_{h}=\left(\mathcal{D}^{\mu} h\right)^{\dagger}\left(\mathcal{D}_{\mu} h\right)-\mu^{2} h^{\dagger} h-\lambda\left(h^{\dagger} h\right)^{2}
$$

Let us consider the potential for the field $h$ :

$$
V_{h}=\mu^{2} h^{\dagger} h+\lambda\left(h^{\dagger} h\right)^{2}
$$

Provided that $\mu^{2}$ is negative, this potential has a continuum of minima, at modulus

$$
v \equiv \sqrt{\frac{-\mu^{2}}{\lambda}}
$$

By suitable gauge rotations, we can choose the minimum to be

$$
h_{0}=\frac{1}{\sqrt{2}}\left(\begin{array}{l}
0 \\
v
\end{array}\right)
$$

Making this choice breaks the standard model group. In addition, the vacuum expectation value (vev) $v$ of the Higgs field will give masses to the quarks and leptons, as seen below.

The physical Higgs particle is now one of the degrees of freedom $\left(h^{\prime}\right)$ in the expansion about the chosen minimum. In one choice of gauge, we can write

$$
h \rightarrow \frac{1}{\sqrt{2}}\left(\begin{array}{c}
0 \\
v+h
\end{array}\right) .
$$

The mass of the Higgs is given by the vev as

$$
M_{h}=\sqrt{-2 \mu^{2}}=\sqrt{2 \lambda v^{2}} .
$$


Now we are ready to discuss the generation of particle mass. First we will consider the bosons. Because the color $S U(3)$ group remains unbroken, the gluons $G_{a}^{\mu}$ remain massless. However, among the four other gauge bosons, only one linear combination can remain massless. We introduce the weak mixing angle $\theta_{\mathrm{w}}$ and define the fields $A^{\mu}$ and $Z^{\mu}$ by

$$
\begin{aligned}
& W_{3}^{\mu}=\cos \theta_{\mathrm{w}} Z^{\mu}+\sin \theta_{\mathrm{w}} A^{\mu}, \\
& B^{\mu}=-\sin \theta_{\mathrm{w}} Z^{\mu}+\cos \theta_{\mathrm{w}} A^{\mu} .
\end{aligned}
$$

Inserting these relations and the Higgs vev into the covariant derivatives in $\mathcal{L}_{h}$, we find that the Lagrangian contains mass terms for the bosons:

$$
\mathcal{L}_{\text {boson mass }}=M_{W}^{2} W_{\mu}^{\dagger} W^{-\mu}+\frac{1}{2} M_{Z}^{2} Z_{\mu} Z^{\mu}
$$

where the masses of the bosons are

$$
\begin{aligned}
& M_{W}=\frac{v g_{2}}{2}, \\
& M_{Z}=\frac{v}{2} \sqrt{g_{1}^{2}+g_{2}^{2}}, \\
& M_{A}=0 .
\end{aligned}
$$

The $A^{\mu}$ is the usual electromagnetic field.

Next we consider the masses of the fermions. First we must define a conjugate of the Higgs field:

$$
\bar{h}=\left(\begin{array}{c}
-h^{* 0} \\
h^{*-}
\end{array}\right)
$$

It has hypercharge opposite to that of $h$. This allows us to add the following gaugeinvariant terms to the Lagrangian:

$$
\mathcal{L}_{\text {Yukawa }}=-g_{l} \bar{l}_{L} h l_{R}-g_{u} \bar{q}_{L} h u_{R}-g_{d} \bar{q}_{L} \bar{h} d_{R}+\text { h.c.. }
$$

By making the substitution of Equation 1.15, we find that the Lagrangian contains

$$
\mathcal{L}_{\text {Yukawa }} \ni-m_{e} \bar{e} e-\frac{m_{e}}{v} \bar{e} e h-m_{u} \bar{u} u-\frac{m_{u}}{v} \bar{u} u h-m_{d} \bar{d} d-\frac{m_{d}}{v} \bar{d} d h .
$$


Notice that because there is no right-handed neutrino in the standard model, the neutrino obtains no mass term. The masses of the fermions are

$$
\begin{aligned}
& m_{e}=\frac{g_{e} v}{\sqrt{2}}, \\
& m_{u}=\frac{g_{u} v}{\sqrt{2}}, \\
& m_{d}=\frac{g_{d} v}{\sqrt{2}} .
\end{aligned}
$$

\subsubsection{Fermion Mixing}

There are known to exist three generations of particles, and there is no reason why the mass and weak eigenstates should be the same. In fact, the propagating fields are mixtures of the weak-interaction eigenstates. We will write this by considering the charged current of the weak interactions:

$$
\begin{aligned}
J_{\text {charged }}^{\mu} & =\left(\begin{array}{lll}
\bar{u} & \bar{c} & \bar{t}
\end{array}\right)_{L} \gamma^{\mu}\left(\begin{array}{c}
d^{\prime} \\
s^{\prime} \\
b^{\prime}
\end{array}\right)_{L} \\
& =\left(\begin{array}{lll}
\bar{u} & \bar{c} & \bar{t}
\end{array}\right)_{L} \gamma^{\mu} V\left(\begin{array}{l}
d \\
s \\
b
\end{array}\right)_{L} .
\end{aligned}
$$

Here $V$ is the Cabibbo-Kobayashi-Maskawa (CKM) matrix. The values of its elements are determined experimentally and by the requirement of unitarity to lie in these ranges [10]:

$$
V=\left(\begin{array}{ccc}
0.9747 \text { to } 0.9759 & 0.218 \text { to } 0.224 & 0.002 \text { to } 0.005 \\
0.218 \text { to } 0.224 & 0.9738 \text { to } 0.9752 & 0.032 \text { to } 0.048 \\
0.004 \text { to } 0.015 & 0.030 \text { to } 0.048 & 0.9988 \text { to } 0.9995
\end{array}\right) \text {. }
$$

The upper $2 \times 2$ submatrix of the CKM matrix is nearly unitary by itself. This motivates us to use a simplification when dealing with only the first two generations of quarks $(d$ and $s)$. In this case we introduce the Cabibbo angle $\theta_{C}$ and use

$$
V_{2 \times 2}=\left(\begin{array}{cc}
\cos \theta_{C} & \sin \theta_{C} \\
-\sin \theta_{C} & \cos \theta_{C}
\end{array}\right),
$$


where $\sin \theta_{C} \simeq 0.22$. In Chapter 4 we will use this simplification.

\subsubsection{Discrete Symmetries}

In addition to the CPT invariance of the field theory, the standard model has some additional accidental discrete symmetries. Because $m_{\nu_{i}}=0$ in the standard model, we see that individual lepton number $\left(L_{i}\right)$ is conserved. Because the quarks mix via the CKM matrix, we cannot say so much about them. However, in the absence of instanton effects we can say that total baryon number $(B)$ is conserved. This is important for the stability of the proton.

\subsubsection{Color Confinement}

The coupling constants of the gauge groups of the standard model run as the energy scale of the interactions changes. It turns out that the coupling of the color $S U(3)$ group runs to a value that is larger than one at low energies. Because of this, quarks exist only in tightly bound hadronic states. The characteristic scale of this binding is the scale $4 \pi \Lambda_{\mathrm{QCD}} \approx 2 \mathrm{GeV}$ at which the coupling becomes nonperturbative. Nevertheless, it is larger than the mass of the strange quark $(\approx 250 \mathrm{MeV})$, so that we can use the approximate $S U(3)$ flavor symmetry of the $u, d$, and $s$ quarks in our treatment of the $D$ mesons in Chapter 4.

\subsection{Grand Unification}

Physicists are always interested in reducing the number of parameters that describe physics while simplifying our understanding of nature. The next simplification beyond the standard model is grand unification. The idea of grand unification is to combine the forces of the standard model into one force. This is accomplished by embedding $G_{\mathrm{SM}}$ in some larger (semisimple) group. The fermions of the standard model are then combined into representations of the unified group. At high energies, 
the interactions are given by the larger gauge symmetry. This larger group must then break down at low energies to the standard model group:

$$
G_{\mathrm{GUT}} \rightarrow G_{\mathrm{SM}}
$$

The breaking scale is typically called $M_{X}$.

The simplest GUT scheme is $S U(5)$ [11]. In it, the standard model group is embedded in $S U(5)$ whose adjoint representation contains twenty-four gauge bosons. These are the usual eight gluons, three weak bosons, and the $B^{\mu}$, plus twelve new bosons $X^{\mu}$ that couple to quarks and leptons. The fermions of one generation are collected into a five-dimensional and a ten-dimensional representation:

$$
\begin{gathered}
\mathbf{5}_{L}=\left(\begin{array}{c}
d_{\mathrm{r}} \\
d_{\mathrm{y}} \\
d_{\mathrm{b}} \\
e \\
\nu
\end{array}\right)_{L}, \\
\mathbf{1 0}_{L}=\frac{1}{\sqrt{2}}\left(\begin{array}{ccccc}
0 & \bar{u}_{\mathrm{b}} & -\bar{u}_{\mathrm{y}} & -u_{\mathrm{r}} & -d_{\mathrm{r}} \\
-\bar{u}_{\mathrm{b}} & 0 & \bar{u}_{\mathrm{r}} & -u_{\mathrm{y}} & -d_{\mathrm{y}} \\
\bar{u}_{\mathrm{y}} & -\bar{u}_{\mathrm{r}} & 0 & -u_{\mathrm{b}} & -d_{\mathrm{b}} \\
u_{\mathrm{r}} & u_{\mathrm{y}} & u_{\mathrm{b}} & 0 & -\bar{e} \\
d_{\mathbf{r}} & d_{\mathrm{y}} & d_{\mathrm{b}} & \bar{e} & 0
\end{array}\right)_{L} .
\end{gathered}
$$

Some of the additional nonstandard couplings are

$$
\mathcal{L}_{\text {int }} \ni g_{5} \bar{d} \gamma_{\mu} u X^{\dagger \mu}+g_{5} \bar{u} \gamma_{\mu} e X^{\mu}+\text { h.c. }
$$

At low energies they are manifest as

$$
\mathcal{L}_{\text {int }} \ni \frac{g_{5}^{2}}{M_{X}^{2}}\left(\bar{d} \gamma_{\mu} u\right)\left(\bar{u} \gamma^{\mu} e\right) .
$$

They can mediate such processes as proton decay, which is forbidden in the standard model. We can estimate the rate as

$$
\Gamma_{p} \simeq \frac{g_{5}^{4}}{M_{X}^{4}} m_{p}^{5}
$$


The experimental limits on the lifetime of the proton [12] suggest that the scale at which GUTs based on $G_{\mathrm{GUT}} \supset S U(5) \supset G_{\mathrm{SM}}$ are broken is

$$
M_{X} \gtrsim 10^{15} \mathrm{GeV}
$$

\section{$1.3 \quad$ Supersymmetry}

Although the coupling constants of the standard model were previously thought to converge at high energies, with today's precise measurements it appears that they require supersymmetry to do so [5]. A supersymmetric threshold between the energy scales of the SM and of the GUT is needed to alter the trajectories of the couplings in the right direction. In this section we will briefly describe the aspects of SUSY that we need in Chapter 2.

SUSY is a symmetry between fermions and bosons. For details we refer the reader to [13] [14]. In SUSY, fields of different spin are combined into supermultiplets. The type of multiplet that we will employ in Chapter 2 is the chiral multiplet. It contains a fermion $f$ of $\operatorname{spin} 1 / 2$, a boson $\tilde{f}$ of spin 0 , and an auxiliary (nonpropagating) field $A_{f}$ of spin 0 :

$$
F=\tilde{f}+\theta f+\theta \theta A_{f}
$$

where $\theta$ is a two-component fermionic coordinate.

The interactions in SUSY are contained in what is called the superpotential $W$. It can contain any term that respects the gauge symmetries and supersymmetry. From it we can derive the interactions of the component fermions and bosons by integrating it over the fermionic components $\theta$ and by solving the equations of motion for the auxiliary fields. The result is that the interactions are

$$
\mathcal{L}_{\text {int }} \ni \sum_{i}\left|\frac{\partial W}{\partial F_{i}}\right|_{F_{i}=\tilde{f}_{i}}^{2}+\left.\sum_{i, j} f_{i} f_{j} \frac{\partial W}{\partial F_{i} \partial F_{j}}\right|_{F_{i}=\tilde{f}_{i}, F_{j}=\tilde{f}_{j}}+\text { h.c. }
$$

Let us consider the results for terms in $W$ with two, three, and four factors. From 
a term in the superpotential of the form

$$
W_{2}=\mu F_{1} F_{2}
$$

the interactions of the fermions and bosons are

$$
\begin{array}{ll}
\mathcal{L}_{\text {int }} \ni \quad \mu f_{1} f_{2}+\text { h.c. } \\
\\
+\mu\left|\tilde{f}_{1}\right|^{2}+\mu\left|\tilde{f}_{2}\right|^{2},
\end{array}
$$

and from a term in the superpotential of the form

$$
W_{3}=\lambda F_{3} F_{4} F_{5}
$$

the interactions of the fermions and bosons are

$$
\begin{aligned}
\mathcal{L}_{\text {int }} \ni \quad & \lambda f_{3} f_{4} \tilde{f}_{5}+\lambda f_{4} f_{5} \tilde{f}_{3}+\lambda f_{5} f_{3} \tilde{f}_{4}+\text { h.c. } \\
& +\lambda\left|\tilde{f}_{3} \tilde{f}_{4}\right|^{2}+\lambda\left|\tilde{f}_{4} \tilde{f}_{5}\right|^{2}+\lambda\left|\tilde{f}_{5} \tilde{f}_{3}\right|^{2} .
\end{aligned}
$$

We will use these in our discussion of nonstandard couplings in the minimal supersymmetric standard model in the next chapter. 


\section{Approximate and Discrete Symme- tries in the MSSM}

The supersymmetric extension of the standard model of particle physics which contains the fewest additional fields is called the minimal supersymmetric standard model (MSSM) [15]. The additional fields required are the supersymmetric partners of the known matter fields and of the gauge and Higgs fields, and an additional Higgs doublet with its partners. In the supersymmetrized model, there are allowed some baryon- and lepton-violating couplings that were not present in the SM. In this chapter we will examine these couplings. We will consider approximate flavor symmetries that can give small natural values to these couplings. These couplings will be found to be smaller than allowed by experiment, except in the case of those that promote proton decay. For this case we will consider discrete symmetries in order to disallow the process. This discussion is based on [1].

\subsection{Couplings in the MSSM}

Here we will review the couplings allowed by the gauge symmetry of the standard model. Since we are only interested in those which violate baryon or lepton number, we will omit the gauge couplings from our discussion. We adopt the usual notation for the superfields in the MSSM ([16], for example). The left-handed doublets are $L_{i}, Q_{i}, H$, and $\bar{H}$. They contain the left-handed lepton doublets $l_{i}$, quark doublets

$q_{i}$ of the SM. Here and below Latin indices run over particle generation. In the MSSM there are two doublets of Higgs scalars, $h$ and $\bar{h}$, contained in the superfields 
$H$ and $\bar{H}$. The left-handed antiparticles $\left(e_{R}\right)_{c},\left(d_{R}\right)_{c}$, and $\left(u_{R}\right)_{c}$ are contained in the chiral superfields $E_{i}, D_{i}$, and $U_{i}$.

In order to give fermions mass, these Yukawa interaction terms are required in the superpotential:

$$
W_{\text {Yuk }}=h_{i j}^{(1)} L_{i} E_{j} H+h_{i j}^{(2)} Q_{i} D_{j} H+h_{i j}^{(3)} Q_{i} U_{j} \bar{H} .
$$

The gauge indices are suppressed. The Yukawa interactions are obtained from the terms of the superpotential in the usual way (see Section 1.3). The masses of the fermions are generated by the terms in Equation 2.1 when the neutral Higgs scalars obtain their vacuum expectation values (vevs). The $W$ mass constrains these vevs $(\langle v\rangle$ and $\langle\bar{v}\rangle)$ to satisfy

$$
\langle v\rangle^{2}+\langle\bar{v}\rangle^{2}=(174 \mathrm{GeV})^{2} .
$$

This value is determined by minimizing the effective potential for $h$ and $\bar{h}$. Including soft symmetry-breaking terms, which are parameterized by the masses $m_{1}$ and $m_{2}$, this potential is

$$
V=m_{1}^{2}|h|^{2}+m_{2}^{2}|\bar{h}|^{2}+\frac{g_{1}^{2}+g_{2}^{2}}{8}\left(|h|^{2}-|\bar{h}|^{2}\right)^{2}
$$

This, however, is not enough, due to an additional $U(1)$ (Peccei-Quinn) symmetry in the potential [17]. Under this symmetry, $h$ and $\bar{h}$ undergo independent phase rotations. When this symmetry is broken by $\langle v\rangle \neq 0 \neq\langle\bar{v}\rangle$ an unwanted axion is produced $[17]$. We can rectify this problem by including the term

$$
W_{H \bar{H}}=\mu H \bar{H}
$$

in the superpotential. It is then likely that the effective potential will also have the supersymmetry-breaking term

$$
\mu m_{3} h \bar{h} .
$$

Here $m_{3}$ is another supersymmetry-breaking mass. The existence of the term in Equation 2.4 will be required in the following. These supersymmetry-breaking parameters and any others will be denoted by $M_{\text {SUSY }}$. Experimental constraints from searches for superpartners [18] require that $M_{\mathrm{SUSY}} \gtrsim 100 \mathrm{GeV}$. 
Although the above couplings are contained in the Standard Model, the supersymmetric theory can contain additional couplings which are also allowed by the gauge structure. A natural theory should contain all allowed couplings. Therefore we will include the following renormalizable terms in the superpotential:

$$
W_{\mathrm{dim}-4}=m_{i} L_{i} H+\lambda_{i j k}^{(1)} L_{i} L_{j} E_{k}+\lambda_{i j k}^{(2)} Q_{i} D_{j} L_{k}+\lambda_{i j k}^{(3)} U_{i} D_{j} D_{k} .
$$

By a suitable redefinition of the $L$ and $H$ superfields the first of these terms can be eliminated. In the following we assume that this redefinition has been performed and that the term in question is no longer present. Note that the antisymmetric contraction of the suppressed $S U(2)$ indices forces the generational indices $i$ and $j$ in the first term and $j$ and $k$ in the fourth to be unequal. All of these terms give interactions that violate either lepton or baryon number or both.

In addition to the terms in Equation 2.6 we can include higher-dimension nonrenormalizable operators. They come about from new physics at some scale $\Lambda$. Those operators of dimension five which violate lepton or baryon number and which are allowed by the gauge symmetry appear in the superpotential as

$$
\begin{aligned}
W_{\mathrm{dim}-5}= & \frac{\kappa_{i j k l}^{(1)}}{\Lambda} Q_{i} Q_{j} Q_{k} L_{l}+\frac{\kappa_{i, k l}^{(2)}}{\Lambda} U_{i} U_{j} D_{k} E_{l}+\frac{\kappa_{i j k}^{(3)}}{\Lambda} Q_{i} Q_{j} Q_{k} H \\
& +\frac{\kappa_{i j k}^{(4)}}{\Lambda} Q_{i} U_{j} E_{k} H+\frac{\kappa_{i j}^{(5)}}{\Lambda} L_{i} L_{j} \overline{H H}+\frac{\kappa_{i}^{(6)}}{\Lambda} L_{i} H \overline{H H} .
\end{aligned}
$$

In the next section we will discuss the experimental constraints on the values of the new couplings in Equations 2.6 and 2.7. Later shall we consider the natural values of the couplings, based on the application of approximate symmetries to the Higgs sector of the theory.

\subsection{Experimental Limits}

Here we will briefly discuss the experimental constraints from the searches for $B$ and $L$-violating processes on the constants $\lambda^{(n)}$ and $\kappa^{(n)} / \Lambda$. The processes that 
we consider are proton decay, neutron-antineutron oscillation, exotic lepton decays, and neutrino oscillations.

\subsubsection{Proton Decay}

If both of $\lambda^{(2)}$ and $\lambda^{(3)}$ are non-zero then lepton and baryon number are not conserved and the proton can decay. A possible decay diagram is shown in Figure 2.1. Using the experimental limit on the proton lifetime [12], we obtain a limit on the couplings:

$$
\sqrt{\lambda^{(2)} \lambda^{(3)}} \lesssim\left(\frac{M_{\mathrm{SUSY}}}{\mathrm{TeV}}\right) \times 10^{-12}
$$

For $M_{\text {SUSY }} \sim 1 \mathrm{TeV}$, we find

$$
\sqrt{\lambda^{(2)} \lambda^{(3)}} \lesssim 10^{-12}
$$

In addition, the first term in Equation 2.7 can contribute to proton decay. In this case a loop involving superpartners such as a Wino is required. An example of a contributing Feynman diagram is given in Figure 2.2. From its contribution to proton decay, we obtain a limit on $\kappa^{(1)}$ :

$$
\frac{\kappa_{1121}^{(1)}}{\Lambda} \sim \frac{\kappa_{1122}^{(1)}}{\Lambda} \lesssim\left(16 \pi^{2}\right) \frac{M_{\mathrm{SUSY}}}{M_{\mathrm{GUT}}^{2}} \sim 10^{-26} \mathrm{GeV}^{-1} .
$$

Proton decay via a diagram involving $\kappa^{(2)}$ that only involves right-handed superfields cannot proceed via Wino exchange unless helicity is flipped in the graph (e.g., Figure 2.3). Each helicity flip adds a factor of $m_{\text {quark }} / M_{\text {SUSY, }}$ and hence no meaningful restraint on $\kappa^{(2)}$ is obtained. By this we mean that if $\kappa^{(2)}$ were to be as large as possible, consistent with perturbative estimates of the decay process, then the estimate of the proton lifetime would be much longer than the experimental limit. Alternatively, a higgsino can be used to complete the graph. Then the suppression contains two factors of $m_{\text {quark }} / v$, where $v$ is either $\langle v\rangle$ or $\langle\bar{v}\rangle$. It is conceivable that the constraint of Equation 2.2 on the vevs may allow such factors to be large, but for the minimal Higgs content, the ratio of $\langle\bar{v}\rangle$ and $\langle v\rangle$ is constrained to be between 1 and $m_{t} / m_{b}[3]$, and hence no meaningful constraint on $\kappa^{(2)}$ can be obtained. 
Figure 2.1: Contribution to proton decay involving $\lambda^{(2)}$ and $\lambda^{(3)}$.

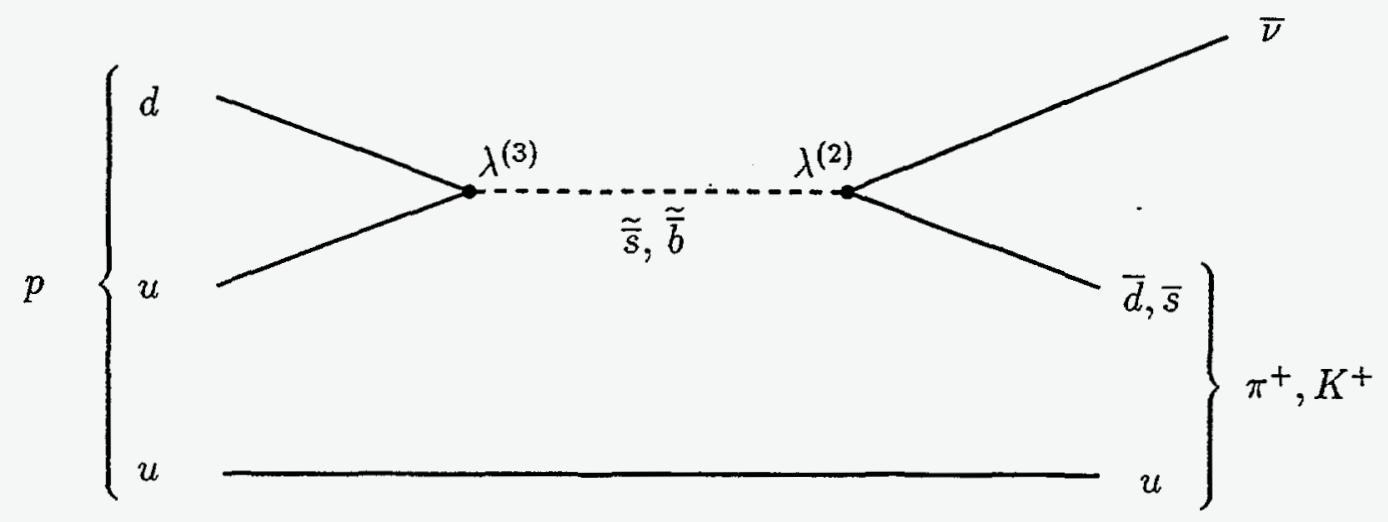

Figure 2.2: Contribution to proton decay involving $\kappa^{(1)}$.

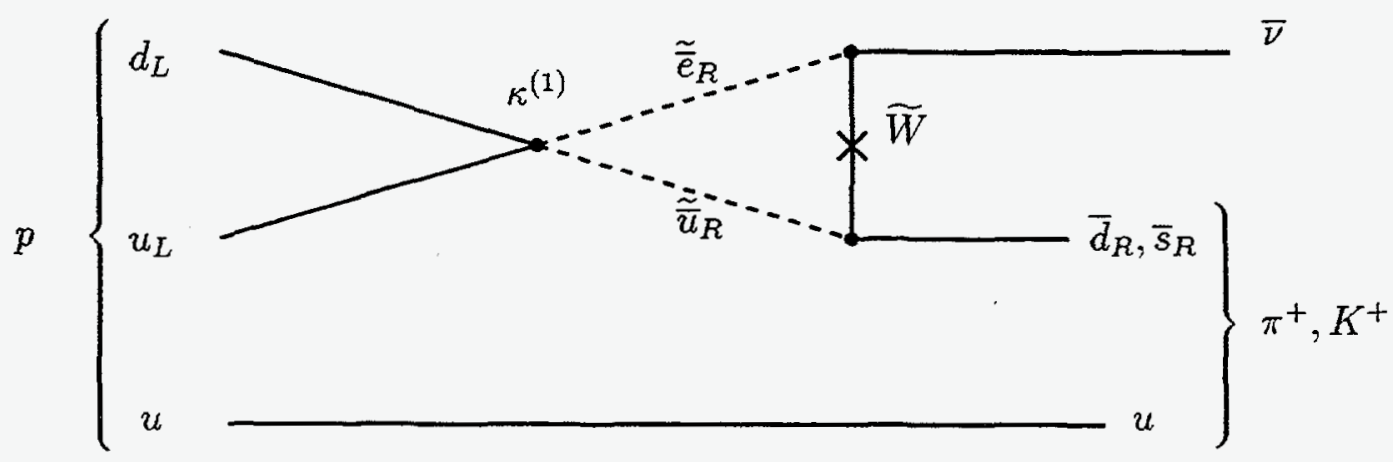


Figure 2.3: $\quad$ Contribution to proton decay involving $\kappa^{(2)}$.

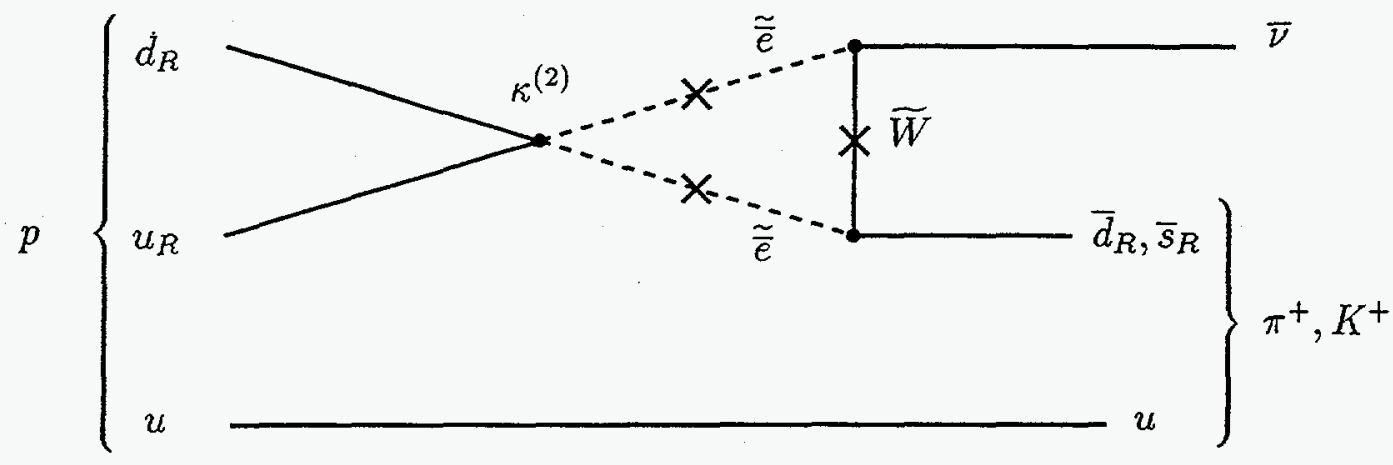

\subsubsection{Neutron-Antineutron Oscillation}

If lepton number is conserved and baryon number is not, then the relevant experimental constraint arises from the absence of neutron-antineutron oscillations. The leading contribution to such oscillations is from a diagram involving two $\lambda^{(3)}$ vertices (Figure 2.4). A limit can be obtained from from the oscillation time of the neutron into an antineutron as obtained by the Fréjus Collaboration results [19]. They find the oscillation time in the environment of a nucleus to be

$$
\tau_{\text {Nucl }} \geq 6.5 \times 10^{31} \mathrm{yr}
$$

Using nuclear corrections [20], the nuclear stability measurements give a free oscillation time of

$$
\tau_{n \leftrightarrow \bar{n}} \geq 1.2 \times 10^{8} \mathrm{~s}
$$

The diagram of leading order in $\lambda^{(3)}$ contributing to neutron-antineutron oscillation is of the type in Figure 2.4a, where the blob represents the insertion of some strangeness- or bottomness-changing interaction. Supposing that the $q \tilde{q}^{\prime} \tilde{g}$ coupling be not diagonal in flavor, [21] and [22] proposed such an interaction mediated by a gluino with a mass insertion (see Figure $2.4 \mathrm{~b}$ ). Here $\xi$ represents an intergenera- 
Figure 2.4a: Contribution to neutron oscillation involving $\lambda^{(3)}$. The blob represents a strangeness- or bottomness-changing interaction (see Figures $2.4 \mathrm{~b}$ and $2.4 \mathrm{c}$ below).

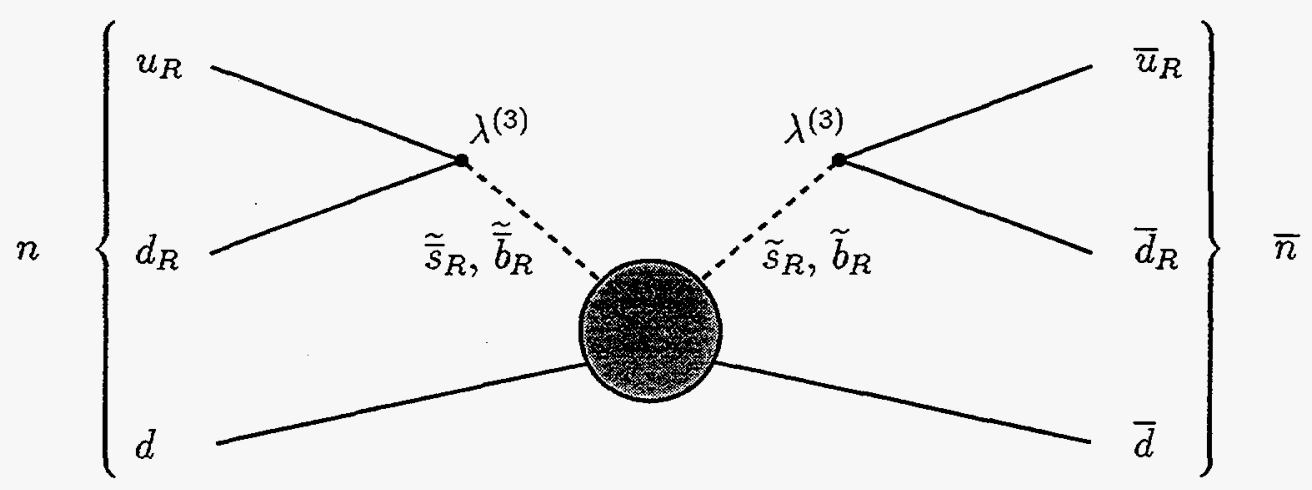

tional coupling. However, $\xi$ must be small because a box diagram containing Figure $2.4 \mathrm{~b}$ must be smaller than the usual box diagram in $\bar{K}^{0}-K^{0}$ mixing. Therefore it is apropriate to consider an insertion of a supersymmetric box subdiagram as in Figure 2.4c [23]. A rough estimate of this process leads to a limit on the couplings

$$
\lambda_{112}^{(3)} \sim \lambda_{113}^{(3)} \lesssim\left(\frac{M_{\mathrm{SUSY}}}{\mathrm{TeV}}\right)^{5 / 2} \times 10^{-4}
$$

No meaningful constraint is obtained for neutron oscillation by $\kappa^{(3)}$ due to the additional powers of $m_{p} / \Lambda$ that are present in this case. 
Figure 2.4b: Possible insertion for the blob of Figure 2.4a [21] [22]. Here $g_{s}$ is the strong coupling and $\xi$ is a factor for intergenerational mixing.

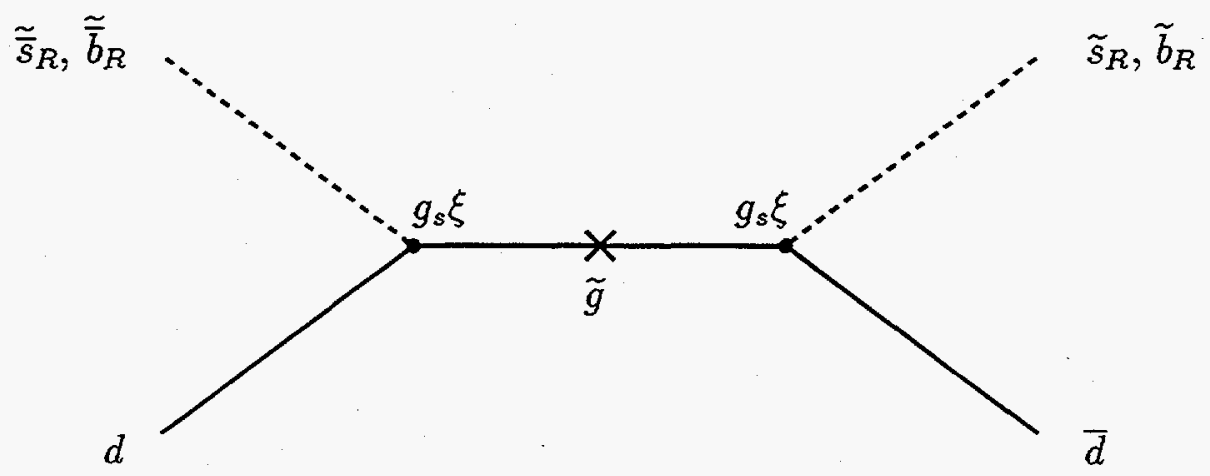

Figure 2.4c: Possible insertion for the blob of Figure 2.4a [23]. Here $g$ is the weak coupling and crosses denote mass insertions needed for helicity change. $U$ is any up-like quark.

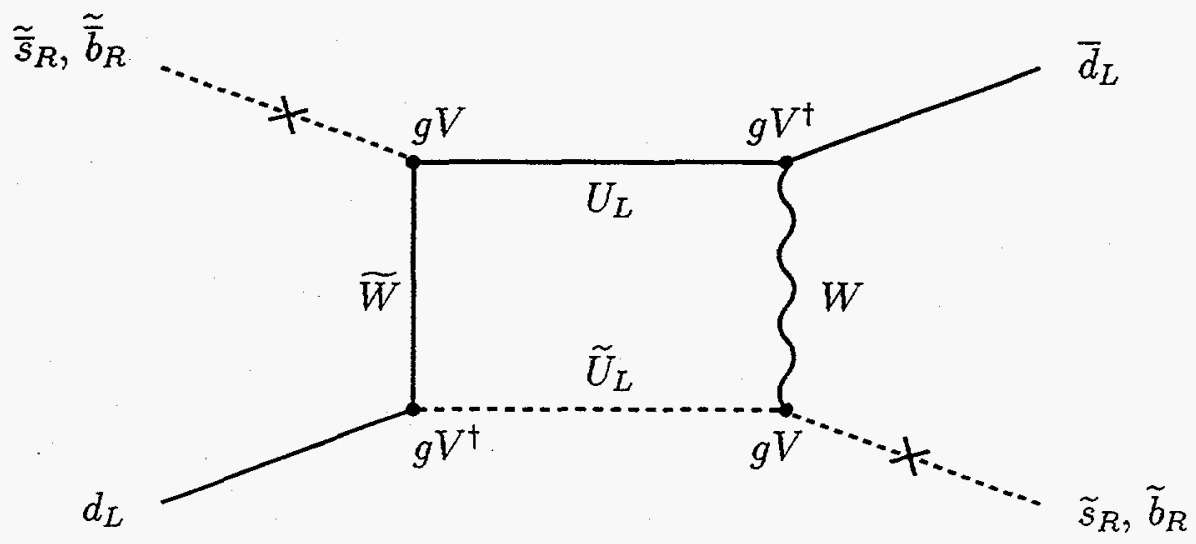


Figure 2.5: Contribution to muon decay involving $\lambda^{(1)}$.

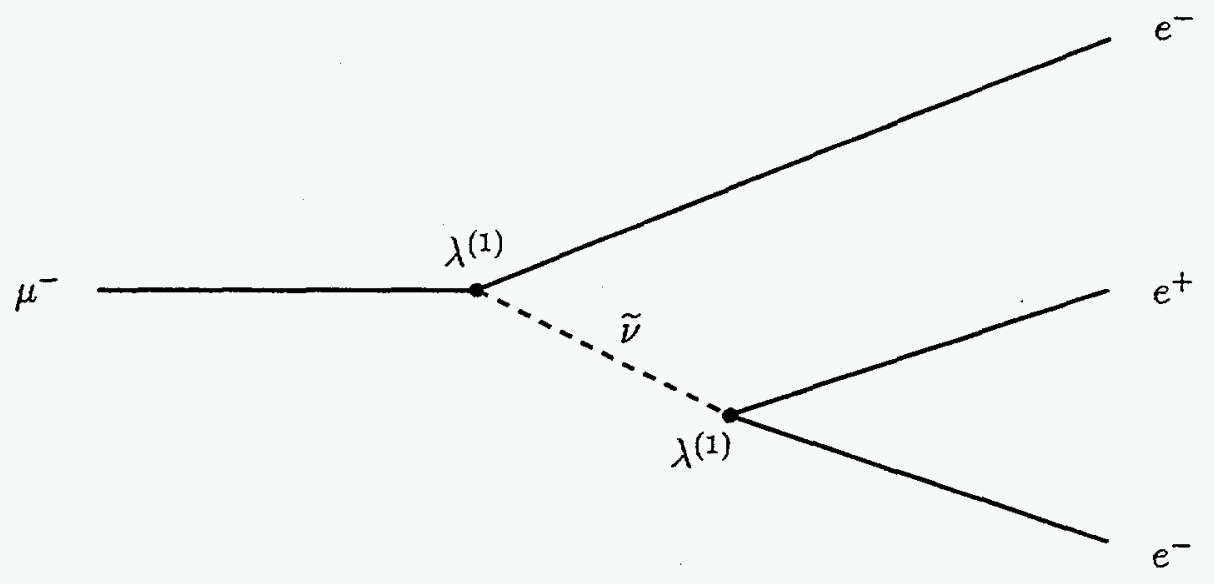

\subsubsection{Exotic Lepton Decays}

If lepton number is violated while baryon number is not, constraints arise from the absence of such processes as $\mu \rightarrow e^{-} e^{-} e^{+}$. This process is allowed if $\lambda^{(1)}$ is present. A tree diagram involving (tau) sneutrino exchange (Figure 2.5), together with the experimental limit on the branching ratio $\operatorname{BR}(\mu \rightarrow 3 e) \lessgtr 1.0 \times 10^{-12}$ from SINDRUM [24], gives this limit on the coupling constants:

$$
\therefore \quad \lambda^{(1)} \lesssim\left(\frac{M_{\text {SUSY }}}{\mathrm{TeV}}\right) \times 10^{-3} .
$$

A similar limit can be obtained using $\mu \rightarrow e \gamma$ and the limit from the Crystal Box [25].

Unfortunately, the decay $\mu \rightarrow e \gamma$ via two vertices involving $\kappa^{(6)}$ and $v e v$ insertions (Figure 2.6, for example) gives no meaningful constraint on $\kappa^{(6)}$.

The term involving $\kappa^{(4)}$ violates lepton number while respecting baryon number. It can mediate exotic decays of the tau such as processes such as $\tau \rightarrow \pi^{0} \mu \nu \chi_{0}$, where $\chi_{0}$ is the lightest supersymmetric partner, (see, for example, Figure 2.7). Using an upper bound on exotic decays of the $\tau$ of about one percent, we obtain no meaningful constraint on $\kappa^{(4)}$ 
Figure 2.6: Contribution to muon decay involving $\kappa^{(6)}$.

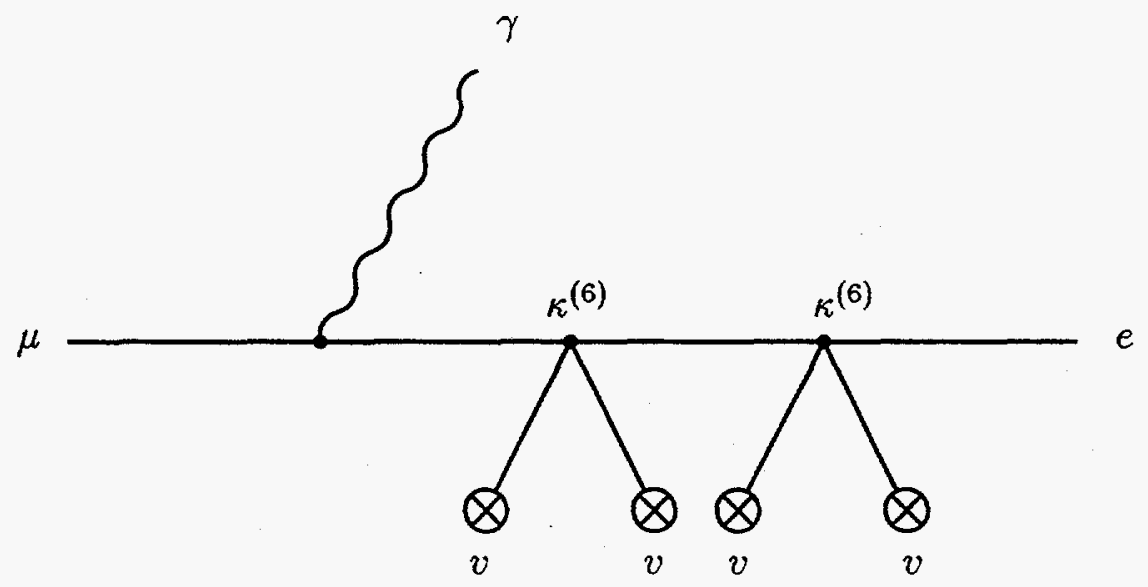

Figure 2.7: Contribution to tau decay involving $\kappa^{(4)}$.

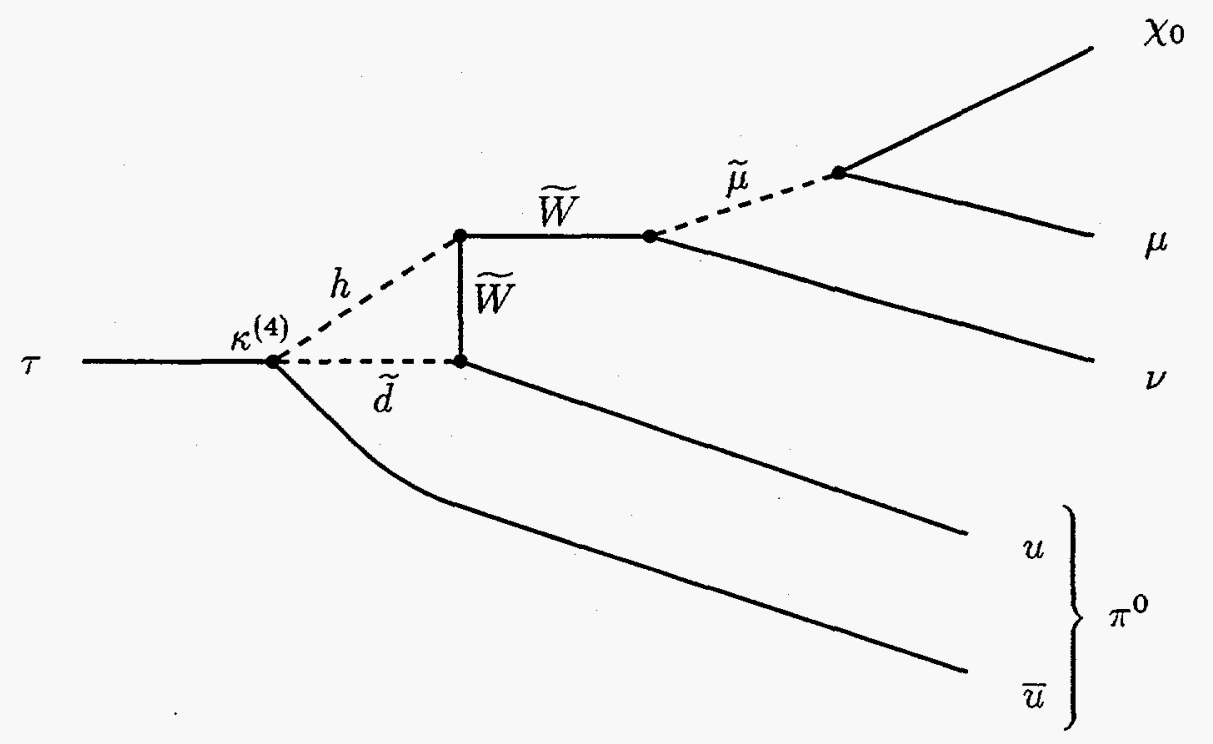




\subsubsection{Neutrino Oscillation}

Once the Higgs doublets have acquired vevs, the term in the superpotential in $\kappa^{(5)}$ takes the form

$$
k_{i j} L_{i} L_{j}
$$

where $k_{i j}$ is a correction to the lepton masses and need not be diagonal in the generations. This term $\kappa^{(5)}$ can therefore induce $\mu \rightarrow e \gamma$, as well as neutrino oscillations. Here experiment [26] constrains the mass difference $\Delta m_{\nu}$ and the mixing angle $\theta$ between the electron and muon neutrino species. The boundary of the excluded region is not a simple function, so we will look at the limit of maximal mixing (i.e., $\sin ^{2} 2 \theta \approx 1$ ). In this limit we find that

$$
\Delta m_{\nu} \sim\left(\frac{\kappa_{12}^{(5)}}{\Lambda}\right)^{2}(250 \mathrm{GeV})^{3} .
$$

This relation puts no useful limit on $\kappa^{(5)}$.

\subsection{Approximate Flavor Symmetries and Natural Values for Couplings}

Fermion masses are (mostly) much smaller than the scale at which the electroweak symmetry breaks and generates their mass. This is indicative of approximate symmetries which involve the separate rotations of the phases of the matter fields [27]. Since the Yukawa coupling constants $h^{(n)}$ in Equation 2.1 are proportional to the quark or lepton masses (divided by the vevs), we expect that the constants $\lambda_{i j k}^{(n)}$ in Equation 2.6 be

$$
\lambda_{i j k}^{(n)} \sim \sqrt{\frac{m_{i} m_{j} m_{k}}{v^{3}}}
$$

where $v$ is taken to be $123 \mathrm{GeV}$, corresponding to $\langle v\rangle \simeq\langle\bar{v}\rangle$ in Equation 2.2. This we call the natural value of the coupling constant $\lambda_{i j k}^{(n)}$. Similarly; the natural value of $\kappa_{i_{1}, \ldots, i_{m}}^{(n)}$ is

$$
\kappa_{i_{1}, \ldots, i_{m}}^{(n)} \sim \prod_{p=1}^{m} \sqrt{\frac{m_{i_{p}}}{v}}
$$


We may now consider the natural values of the couplings which are constrained by experiment in Section 2.2. Clearly, our prescription for assigning natural values to the coupling constants gives the largest couplings for the vertices involving thirdgeneration (s)particles. For example, proton decay is most likely to occur to the final state $K \mu$ or $K \nu_{\mu}$. The natural values for the leading contributions to proton decay are then

$$
\begin{gathered}
\lambda_{121}^{(2)} \sim \lambda_{112}^{(3)} \sim 10^{-5}, \\
\kappa_{1122}^{(1)} \sim 10^{-7} .
\end{gathered}
$$

For neutron oscillation also we must consider $\lambda_{112}^{(3)}$, whose natural value is given above, and $\lambda_{113}^{(3)}$, whose natural value is

$$
\lambda_{113}^{(3)} \sim 3 \times 10^{-5}
$$

For $\mu$ decay to $3 e$ we have the natural value

$$
\lambda_{123}^{(1)} \sim 10^{-5} .
$$

As we can see, the natural values for the couplings are well below the current experimental limits, with the exception of those involved in proton decay. The combination of $\lambda^{(2)} \lambda^{(3)}$ in proton decay has a natural value much larger than the experimental limit. If the scale of new physics $\Lambda$ that appears in the dimension-five couplings is near the GUT scale, then $\kappa^{(1)}$ may also have a natural value that is not allowed by experiment. Hence if either but not both lepton and baryon number is violated by terms having natural strength, the resulting rates will be too small to have been seen in current experiments.

If both of $\lambda^{(2)}$ and $\lambda^{(3)}$, or $\kappa^{(1)}$, are present with their natural values then fast proton decay would occur. We now turn to the possible discrete symmetries that would forbid these terms [28]. 


\subsection{Discrete Symmetries and Proton Decay}

Motivated by the need to suppress proton decay, we consider discrete $Z_{N}$ symmetries, possibly resulting from the breaking of some continuous $U(1)$ symmetry. An analysis of discrete symmetries was given in [28]. A similar analysis regarding proton decay is in [29]. The possible symmetries will be characterized by the charges of the chiral superfields under those symmetries, and by the terms in Equations 2.6 and 2.7 that are allowed.

We write the charges on the superfields as a vector:

$$
\vec{\alpha}=\left(\alpha_{Q}, \alpha_{U}, \alpha_{D}, \alpha_{L}, \alpha_{E}, \alpha_{H}, \alpha_{\bar{H}}\right),
$$

where the transformation of a superfield $\Phi$ with $Z_{N}$ charge $\alpha_{\Phi}$ under the discrete symmetry is

$$
\Phi \rightarrow e^{\frac{2 \pi i}{N} \alpha_{\Phi}} \Phi .
$$

Due to weak hypercharge invariance of all of the terms in the superpotential, we can assign the charge of one field (choose $Q$ ) to be zero. because we require the presence of the Yukawa couplings in Equation 2.1, we have the following conditions on the $Z_{N}$ charges of the Higgs:

$$
\begin{array}{ll}
\alpha_{H}=-\alpha_{Q}-\alpha_{D} & (\bmod N), \\
\alpha_{H}=-\alpha_{L}-\alpha_{E} & (\bmod N), \\
\alpha_{\bar{H}}=-\alpha_{Q}-\alpha_{U} & (\bmod N) .
\end{array}
$$

The first two of Equations 2.25 lead to a condition on the charges of the matter superfields:

$$
\alpha_{Q}+\alpha_{D}=\alpha_{L}+\alpha_{E} \quad(\bmod N) .
$$

These conditions reduce the number of independent charges to three. Thus we can choose a convenient basis in which the charge of any field is given in terms of three 
integers $(m, n$ and $p)$.

$$
\begin{aligned}
& \vec{\alpha}_{R}=(0,-1, \quad 1, \quad 0, \quad 1,-1, \quad 1), \\
& \vec{\alpha}_{A}=(0, \quad 0,-1,-1, \quad 0, \quad 1, \quad 0) \text {, } \\
& \text { and } \vec{\alpha}_{L}=(0, \quad 0, \quad 0,-1, \quad 1, \quad 0,0) \text {. }
\end{aligned}
$$

The total charges may be written as

$$
\vec{\alpha}=m \vec{\alpha}_{R}+n \vec{\alpha}_{A}+p \vec{\alpha}_{L}
$$

Notice that the last of the vectors in Equation 2.27 is equivalent to antilepton number.

We now examine the symmetries that will allow which of the dimension-four and -five couplings of Equations 2.6 and 2.7. That the L-violating couplings $L L E$ and $Q D L\left(\lambda^{(1)}\right.$ and $\left.\lambda^{(2)}\right)$ be allowed is the condition

$$
m-2 n-p=0 \quad(\bmod N)
$$

That the B-violating coupling $U D D\left(\lambda^{(3)}\right)$ be allowed is the condition

$$
m-2 n=0 \quad(\bmod N)
$$

That the term $Q Q Q L\left(\kappa^{(1)}\right)$ be allowed is the condition

$$
n-p=0 \quad(\bmod N)
$$

Requiring that the Higgs mass term of Equation 2.4 be allowed implies that

$$
\begin{aligned}
\alpha_{H}+\alpha_{\bar{H}} & =0(\bmod N) \\
\text { or } \quad & =0(\bmod N)
\end{aligned}
$$

Since we expect that the discrete symmetries will be remnants of a gauged $U(1)$ symmetry, we may also impose anomaly-cancellation conditions on the charges of the superfields [30]. The transformation in Equation 2.24 tells us that the $U(1)$ charges are

$$
q_{i}=\alpha_{i}+\beta_{i} N
$$


where the $\beta_{i}$ are integers. The anomalies of the $U(1)$ symmetry will cancel if constraints on the charges of the various fermions in the theory are satisfied. For example, the $Z_{N}^{3}$ anomaly-cancellation condition is

$$
\sum_{i} q_{i}^{3}=r N+s \frac{N^{3}}{8} .
$$

Where the sum on the left side of this equation runs over all of the quark, lepton and Higgsino fields of charge $q_{i}$. The right-hand side of this equation arises from fermions that have mass of order of the scale at which the $U(1) \rightarrow Z_{N}$ breaking takes place. Such fermions can either have Dirac or Majorana masses. In order to have a Majorana mass a particle must have a $Z_{N}$ charge of 0 or $N / 2$. The integer $s$ is the number of these Majorana fermions with charge $N / 2$. Two chiral fermions of charge $a_{j}$ and $a_{j}^{\prime}$ can couple to generate one Dirac particle provided that $a_{j}+a_{j}^{\prime}=z_{j} N$, where $z_{j}$ is an integer. In this case

$$
r=\sum_{\text {Dirac pairs }} z_{j}\left(-N^{2} z_{j}^{2}+3 a_{j} N z_{j}-3 a_{j}^{2}\right) .
$$

The triangle anomaly-cancellation conditions of involving both $Z_{N}$ and $U(1)_{Y}$ are $\left(Z_{N^{-}} U(1)-U(1)\right.$ and $\left.Z_{N^{-}}^{2} U(1)\right)$

$$
\begin{aligned}
& \sum_{i} y_{i}^{2} q_{i}=-\sum_{\text {heavy pairs }}\left(a_{j}+a_{j^{\prime}}\right) y_{j}^{2} \\
& \sum_{i} y_{i} q_{i}^{2}=-\sum_{\text {heavy pairs }}\left(a_{j}^{2}-a_{j^{\prime}}^{2}\right) y_{j}
\end{aligned}
$$

where $y_{i}$ are the $U(1)_{\mathrm{Y}}$ charges. Note that the Majorana fermions (if any) must have $y_{j}=0$, and the pairs of Dirac fermions have $y_{j}=-y_{j^{\prime}}$. The $Z_{N^{-}} S U(M)-S U(M)$ anomaly-cancellation condition is

$$
\sum_{i} T_{i} q_{i}=-\sum_{\text {heavies }} T_{j} q_{j},
$$

where $T_{i}$ are the Casimir operators of $S U(M)$. (normalized such that the value on the fundamental representation is $1 / 2$ ), and the right-hand side is for heavy fermions. Note that only Majorana particles in real representations of $S U(M)$ can contribute. The Dirac fermions cancel in pairs. The $Z_{N}$-gravity-gravity anomalycancellation condition is

$$
\sum_{i} q_{i}=r^{\prime} N+s^{\prime} \frac{N}{2}
$$


where $r^{\prime}$ and $s^{\prime}$ have the same origins as $r$ and $s$ in Equation 2.34. In terms of the exponents $m, n$, and $p$ in Equation 2.28 we write these conditions for the $Z_{N^{-}}$ gravity-gravity, $Z_{N^{-}} S U(2)-S U(2)$, and $Z_{N^{-}} S U(3)-S U(3)$ anomalies as

$$
\begin{aligned}
(m-5 n-p) N_{G}+2 n N_{H} & =t N+s \frac{N}{2} \\
-(p+n) N_{G}+n N_{H} & =t^{\prime} N \\
-n N_{G} & =t^{\prime \prime} N .
\end{aligned}
$$

Here $N_{G}$ denotes the number of generations of quarks and leptons, and $N_{H}$ the number of sets of $H$ and $\bar{H}$. The integers $t, t^{\prime}$, and $t^{\prime \prime}$ depend on the $\beta_{i}$ in Equation 2.33 and on $\sum_{\text {heavies }}$ and $r^{\prime}$ in Equations 2.37 and 2.38:

$$
\begin{aligned}
t & =\sum_{i} \beta_{i}+r^{\prime} \\
t^{\prime} & =\sum_{i} \beta_{i}-\frac{1}{N} \sum_{\text {heavies }} T_{j}^{S U(2)} q_{j}, \\
t^{\prime \prime} & =\sum_{i} \beta_{i}-\frac{1}{N} \sum_{\text {heavies }} T_{j}^{S U(3)} q_{j} .
\end{aligned}
$$

Note that if $N$ is odd, then we must take $s$ to be even, and the last term in the first of Equations 2.39 may be absorbed into $t N$. Note also that as a result of Equation 2.32, the Higgs superfields do not contribute to any of the anomalies provided that $\beta_{H}$ and $\beta_{\bar{H}}$ are zero, which we now assume.

If we wish to have a discrete symmetry which is anomaly-free for each generation, we can set $N_{G}$ to 1 in Equation 2.39. Only one $Z_{2}$ symmetry, $R_{2}(m=1$, $n=p=0$ ), survives. The anomaly cancellation for this symmetry requires the addition of one heavy Majorana particle with $Z_{N}$ charge 1 per generation. Its restrictions on the superpotential is the same as that of conventional $R$-parity, i.e., it forbids all of the B-and L-violating terms in Equation 2.6. However, it still allows the dangerous term in Equation 2.7 involving $\kappa^{(1)}$. If we extend this case to arbitrary $N_{G}$, then we find an anomaly-free $R_{2}$ symmetry, with the addition of $N_{G}$ heavy Majorana fermions.

In the case of $Z_{3}$, the anomaly conditions of $Z_{N} \times S U(2) \times S U(2)$ and $Z_{N} \times$ $S U(3) \times S U(3)$ are trivially satisfied for the the phenomenologically relevant case $N_{G}=3$ and $N_{H}=1$ for $n=0$ and $m$ and $p$ unconstrained by appropriate choices 
of the $\beta_{i}$. So we can consider the inequivalent set $R_{3}, L_{3}, R_{3} L_{3}$, and $R_{3} L_{3}^{2}$. When we require that the other anomaly conditions be satisfied only $R_{3} L_{3}$ survives. Additional heavy fields are required to achieve anomaly cancellation. Several choices are possible. For example, choosing the $\beta_{i}$ so that the charges of $(Q, U, D, L, E$, $H, \bar{H})$ are $(0,-1,1,-1,2,-1,1)$, We need the following set of heavy fields: an $\mathrm{SU}(3)$-singlet $\mathrm{SU}(2)$-doublet Dirac pair with $\left(Z_{N}, U(1)_{Y}\right)$ charges $(1,1)$ and $(2,-1)$, an $S U(3) \times S U(2)$-singlet Dirac pair with $\left(Z_{N}, U(1)_{Y}\right)$ charges $(-1,2)$ and $(-2,-2)$, and an $S U(3) \times S U(2)$-singlet Dirac pair with $\left(Z_{N}, U(1)_{Y}\right)$ charges $(0,0)$ and $(-3$, $0) .^{1}$

This symmetry satisfies our requirements regarding proton decay. That is, it allows the coupling $Q D L$, but not $U D D$. Furthermore, the term in $\kappa^{(1)}$ is also disallowed. That L-violating terms may still be allowed is not troublesome in light of the naturalness assumptions on their coupling constants, as seen in Section 2.3.

Note that for $N$ higher than 3, the conditions of Equations 2.39 require additional generations of particles. We will therefore not consider them.

\subsection{Generalized $R$-Parities}

Discrete symmetries in which the fermionic measure $d \theta^{2}$ also carries a $Z_{N}$ charge can also be imposed on supersymmetric theories:

$$
\mathrm{d} \theta^{2} \rightarrow e^{-\frac{2 \pi i}{N} \alpha_{W}} \mathrm{~d} \theta^{2}
$$

Under these generalized $R$-parities the superpotential must transform with the opposite charge:

$$
W_{F} \rightarrow e^{\frac{2 \pi i}{N} \alpha_{W}} W_{F}
$$

Such discrete symmetries cannot arise as subgroups of gauge symmetries and

\footnotetext{
${ }^{1}$ This symmetry is equivalent to the "baryon parity" of [28]. Note, however, that we disagree with the claim of [28] that the symmetry is anomaly-free with the minimal particle content of the standard model.
} 
hence we can have no requirement of anomaly cancellation. We would expect therefore that there would be many more possible symmetries [28]. Here we will discuss the case of $Z_{2}$.

In order that the mass terms for the quarks and leptons (Equation 2.1) be allowed in the superpotential, the following must be satisfied:

$$
\begin{array}{ll}
\alpha_{Q}+\alpha_{D}+\alpha_{H}=1 & (\bmod 2), \\
\alpha_{Q}+\alpha_{U}+\alpha_{\bar{H}}=1 & (\bmod 2), \\
\alpha_{L}+\alpha_{E}+\alpha_{H}=1 & (\bmod 2) .
\end{array}
$$

In order to retain the Higgs mass term in Equation 2.4, we require

$$
\alpha_{H}+\alpha_{\bar{H}}=1 \quad(\bmod 2),
$$

As before, we can select a basis

$$
\begin{aligned}
\vec{\alpha}_{R} & =(0,1,1,0,1,0,0), \\
\vec{\alpha}_{A} & =\left(\begin{array}{lllll}
0, & 0,1, & 1, & 0, & 0,1
\end{array}\right) \\
\text { and } \vec{\alpha}_{L} & =\left(\begin{array}{lllll}
0, & 0, & 0,1, & 1, & 1
\end{array}\right)
\end{aligned}
$$

so that the total charges can be written as

$$
\vec{\alpha}=m \vec{\alpha}_{R}+n \vec{\alpha}_{A}+p \vec{\alpha}_{L} .
$$

Using Equation 2.44, we see that the $H \bar{H}$ term requires $n=1$ The L-violating conditions that allow $L L E$ and $Q D L$ are

$$
m-2 n-p=1 \quad(\bmod 2) .
$$

And the B-violating condition that allows $U D D$ becomes

$$
m-2 n=1 \quad(\bmod 2) .
$$

For the $Q Q Q L$ term to be allowed we need

$$
n+p=1 \quad(\bmod 2) .
$$


Since these equations can be solved by $p=0, n=1, m=1$, we are able to find a $Z_{2}$ symmetry that is phenomenologically unacceptable, in contrast to the case of Section 2.4. Conventional $\mathrm{R}$-parity is equivalent to the case $n=1, m=p=0$, which forbids all of the dimension-four B-and L-violating terms.

\subsection{Conclusions}

We have examined possible terms that are allowed by gauge invariance in the supersymmetric standard model and that violate lepton and baryon number. The experimental constraints on these terms have been discussed. Using an assumption on the size of these couplings that is inspired by the size of the couplings that give quark and lepton masses, we have established natural values for the strength of these couplings. Only those terms that can lead to proton decay have natural values that are larger than the experimental constraints.

There is only one possible anomaly-free $Z_{2}$ discrete symmetry that can exist without the addition of new particles having masses of order of the electroweak scale. This symmetry requires the existence of heavy Majorana fermions and forbids all of the renormalizable $\mathrm{B}$ - and L-violating terms. However, it allows the term $Q Q Q L$ which produces proton decay at an unacceptable rate and does not constrain the number of generations. It is equivalent to conventional R-parity [15].

There is only one possible anomaly-free $Z_{3}$ discrete symmetry that can exist without the addition of new particles having masses of order the electroweak scale. This symmetry requires that there be three generations of quarks and leptons and that there exist heavy fermions. It forbids all of the renormalizable $\mathrm{B}$-violating terms, as well as the term $Q Q Q L$. Lepton number is violated in this case. Decays such as $\mu \rightarrow e \gamma$ are expected to occur at rates below the current limits if the couplings have the values that we expect. 


\section{$3 \quad S U(3)$ Coupling Coefficients}

The Clebsch-Gordan coefficients ${ }^{2}$ of $S U(3)$ are useful in calculations involving baryons and mesons, as well as in calculations involving arbitrary numbers of quarks. Tables of these coefficients and their isoscalar factors have been compiled in the past [31] [32] [33] [34] [35], and programs have been distributed that calculate these coefficients [36] [37] [38]. However, our calculations require additional tables. For example, in the decay of a charmed quark (c) to one of the light $(u, d, s)$ quarks, the transition involves operators in the representation $\mathbf{1 5}$, whose tables are not found in the literature. In this chapter we will describe the calculation and present the coupling coefficients that are needed for our study of the charm-changing interactions in Chapter 4. A computer program was used to handle the complex task at hand. It is described herein.

Consider the product of two irreducible representations,

$$
\mathbf{r} \otimes \mathbf{r}^{\prime}=\mathbf{R}_{1} \oplus \mathbf{R}_{\mathbf{2}} \oplus \mathbf{R}_{\mathbf{3}} \oplus \ldots
$$

We will denote the Clebsch-Gordan coefficients in this expansion by $\left\langle\mathbf{R}_{\mathbf{k}} Y_{k} I_{k} I_{3 k}\right|$ $\left.\mathbf{r} y i i_{3} \mathbf{r}^{\prime} y^{\prime} i^{\prime} i_{3}^{\prime}\right\rangle$. The usual definition of the coefficients is given by the equation

$$
\mathbf{r}\left(y, i, i_{3}\right) \times \mathbf{r}^{\prime}\left(y^{\prime}, i^{\prime}, i_{3}^{\prime}\right)=\sum\left\langle\mathbf{R}_{\mathbf{k}} Y I I_{3} \mid \mathbf{r} y i i_{3} \mathbf{r}^{\prime} y^{\prime} i^{\prime} i_{3}^{\prime}\right\rangle \mathbf{R}_{\mathbf{k}}\left(Y, I, I_{3}\right) .
$$

Here $\mathbf{R}_{\mathbf{k}}\left(Y, I, I_{3}\right)$ denotes the state in representation $\mathbf{R}_{\mathbf{k}}$ with hypercharge, total isospin, and third component of isospin of $Y, I$, and $I_{3}$, where $\mathbf{R}_{\mathbf{k}}$ is in the span of $\mathbf{r}$ and $\mathbf{r}^{\prime}$. The sum is over all such states in all such representations.

\footnotetext{
${ }^{2}$ The Clebsch-Gordan coefficients are also called vector coupling coefficients or Wigner coefficients in the literature; the isoscalar factors are also called Racah coefficients.
} 
This chapter takes a utilitarian approach to the calculation. We will introduce only the concepts that are needed to arrive at our results. Readers interested in a deeper theoretical background are directed to [31] [39] [40] [41].

The chapter is organized as follows. In Section 3.1 we introduce the necessary definitions and operations. Section 3.2 contains a discussion of the construction of the irreducible representations. The method of the calculation itself is described in Section 3.3. A very useful way of contracting the tables is the extraction of isoscalar factors based on the Clebsch-Gordan coefficients of the smaller group $S U(2)$. This is discussed in Section 3.4. There we also present some symmetry relations that allow us to omit some tables. The actual computer program is outlined in Section 3.5. Finally, the tables of isoscalar factors are presented in the last section.

\subsection{Basic Concepts}

In this section we present the basic definitions and operations that are needed in order to construct the representations of $S U(3)$ and to determine the ClebschGordan coefficients in their products.

Representations are written as tensors. These tensors carry an arbitrary number of indices that run over the values $u=1, d=2, s=3$. We have chosen to name

these values by the quark names, since it is the $S U(3)$ symmetry among the light quarks that interests us. It is conventional to write the indices as upper indices when they correspond to $u, d$, and $s$, and as lower indices when they represent their conjugates $\bar{u}, \bar{d}$, and $\bar{s}$. Nevertheless, one can use the totally antisymmetric tensor $\epsilon^{i j k}$ to raise all lower indices:

$$
A_{x y z \ldots}^{i j a b c \ldots}=\epsilon^{i j k} A_{k x y z \ldots}^{a b c \ldots .} .
$$

Repeated indices are summed. We choose to write tensors with only upper indices. In order to simplify the notation, tensors will often be represented by the values of their indices. See the example in Equation 3.5. 
Isospin $S U(2)$ is a subgroup of $S U(3)$ which commutes with the hypercharge $U(1)$ (this is the strong hypercharge, not the weak hypercharge introduced in Chapter 1). Therefore states in representations can be taken as those tensors with definite hypercharge and isospin. They are labelled by their quantum numbers as $\mathbf{R}\left(Y, I, I_{3}\right)$. The hypercharge of a state is just the sum of the hypercharges corresponding to the indices that it carries, where the hypercharges of $u, d, s$ are $\frac{1}{3}, \frac{1}{3},-\frac{2}{3}$, respectively. The same is true for the third component of isospin where $u, d, s$ carry $\frac{1}{2},-\frac{1}{2}, 0$. As an example familiar to particle physicists, consider the highest-weight state of the octet. This state corresponds to the $K^{+}$in the pseudoscalar octet, or to the proton in the baryon octet. As a tensor, it is written

$$
8 \text { (h.w. })=8\left(1, \frac{1}{2}, \frac{1}{2}\right)=A_{s}^{u} \dot{=} u \bar{s} \text {. }
$$

We raise the lower index to obtain a properly normalized state

$$
8\left(1, \frac{1}{2}, \frac{1}{2}\right)=A_{s}^{u}=\frac{1}{\sqrt{2}}\left(A^{u u d}-A^{u d u}\right)=\frac{1}{\sqrt{2}}(u u d-u d u) .
$$

(Actually, there is a two-dimensional space of octets carrying three indices. Their highest-weight states are spanned by $\frac{1}{\sqrt{2}}(u u d-u d u)$ and $\frac{1}{\sqrt{6}}(u u d+u d u-2 d u u)$. We have chosen one of them.)

A state written as a tensor with $n$ upper indices can also be viewed as an $n$ dimensional vector. It then seems natural to define both inner and outer products of states. The inner (scalar) product of two states with the same number of indices is defined by

$$
A^{i \ldots j} \cdot B^{k \ldots l}=A^{i \ldots j} B^{i \ldots j}
$$

where repeated indices are summed. The outer (tensor) product can be defined for two states with not necessarily the same number of indices. It is

$$
A^{i \ldots j} \times B^{k \ldots l}=C^{i \ldots j k \ldots l},
$$

where the components of $C$ are products of components of $A$ and $B$ :

$$
C^{i \ldots j k \ldots l}=\left(A^{i \ldots j}\right)\left(B^{k \ldots l}\right)
$$


To clarify this last definition, let us consider the highest-weight state of the 8 and the highest-weight state of the $\overline{\mathbf{3}}$, which is

$$
\overline{\mathbf{3}}(\text { h.w. })=\overline{\mathbf{3}}\left(\frac{2}{3}, 0,0\right)=\frac{1}{\sqrt{2}}(u d-d u) .
$$

Their outer product is

$$
\begin{aligned}
8\left(1, \frac{1}{2}, \frac{1}{2}\right) \times \overline{\mathbf{3}}\left(\frac{2}{3}, 0,0\right) & =\frac{1}{2}(u u d-u d u) \times(u d-d u) \\
& =\frac{1}{2}(u u d u d-u u d d u-u d u u d+u d u d u) \\
& =\overline{\mathbf{1 5}}\left(\frac{5}{3}, \frac{1}{2}, \frac{1}{2}\right) .
\end{aligned}
$$

There remains one more concept that must be discussed before we begin to construct the representations. It is the idea of the operators of $S U(3)$. There are eight operators in the group. Two of them correspond to the hypercharge and third component of isospin. The other six correspond to the raising and lowering operators (the "ladder" operators) in the three possible $S U(2)$ subgroups. They are customarily called the $T$-, $U$-, and $V$-spin subgroups, and their ladder operators are denoted $T^{ \pm}, U^{ \pm}$, and $V^{ \pm}$. Implementing the ladder operators in a practical way is our concern for the moment. Their operation on a state represented by a tensor of indices is manifest in the raising or lowering of the values of each index separately and adding the results into a new tensor. Tensors are renormalized after the operations. We define the direction of raising to be

$$
\begin{aligned}
& T^{+}: d \rightarrow u, \\
& U^{+}: s \rightarrow d, \\
& V^{+}: s \rightarrow u .
\end{aligned}
$$

As an example, let us apply $T^{-}$to the octet's highest-weight state given in Equation 3.5 :

$$
\begin{aligned}
T^{-} \frac{1}{\sqrt{2}}(u u d-u d u) & =\frac{N}{\sqrt{2}}(d u d+u d d-d d u-u d d) \\
& =\frac{N}{\sqrt{2}}(d u d-d d u) .
\end{aligned}
$$

In this case, since these two states form an isospin doublet, the normalization factor $N$ is simply equal to 1 . 


\subsection{Irreducible Representations}

Armed with the above ideas, we are now ready to discuss the construction of the irreducible representations of $S U(3)$. One way to define an irreducible representation is as a set of states that is complete and closed under the operators of the group. By complete we mean that from one state in the representation, any other state in it can be reached by (successive) ladder operations. By closed we mean that states outside the representation cannot be reached by (successive) ladder operations. It follows that we can construct all the states of a representation from one state by using the ladder operators. That one state is typically chosen to be the highestweight state, defined as the state of highest isospin. For this work, however, we consider the highest-weight state to be that which satisfies the conditions

$$
\begin{aligned}
& \left.T^{+} \mid \text {h.w. }\right\rangle=0, \\
& \left.U^{+} \mid \text {h.w. }\right\rangle=0, \\
& \left.V^{+} \mid \text {h.w. }\right\rangle=0,
\end{aligned}
$$

where the $(+)$ directions are given in Equation 3.11.

Therefore a representation of $S U(3)$ can be seen as a collection of $S U(2)$ multiplets (henceforth called isomultiplets), each at a specific hypercharge. For each isomultiplet we have chosen to adopt the Condon-Shortley phase conventions [42]. This means that the eigenvalues of the isospin-raising and -lowering operators (the $T$-spin operators) are real and positive. It remains to specify the relative phases between the isomultiplets of a given representation and the overall phases of representations in the Clebsch-Gordan series. Overall phases of representations will be discussed in Section 3.3. For the relative phases between isomultiplets in a given representation, we have adopted the de Swart phase convention [31]. It corresponds to requiring that the eigenvalues of the $V$-spin operators be real and positive. This is simply an extension of the Condon-Shortley convention to the $V$-spin operators. It is not possible to simultaneously require that the eigenvalues of all operators be positive. Because these conventions may differ from the phase conventions of other 
authors, we caution the reader not to mix the usage of our tables with theirs.

Representations can be labelled by the usual pair of integers $(p, q)$. These integers are the number of ladder operations between the corners of the representation's weight diagram. The names of representations are given by [43].

In some cases, there will exist more than one state in a representation at a given $y$ and $i_{3}$. These states are distinguished by different total isospins. Finding the two or more states at a given site in weight space (the space with coordinates $y$ and $i_{3}$ ) is done by applying different sequences of the ladder operators on the highest-weight state. The results are in general not states with definite total isospin $i$. We handle this problem as follows: At a given hypercharge, the highest-isospin state is not degenerate. From it we construct its complete isomultiplet. The remaining isomultiplets are then forced to be orthogonal to this isomultiplet. We repeat the process on the remaining states and find the isomultiplet of next-highest total isospin: This continues until all states at the given hypercharge have been decomposed into states of definite $i$ and $i_{3}$.

\subsection{Tensor Decomposition}

In this section we will describe the method by which the product of two irreducible representations is decomposed and the Clebsch-Gordan coefficients are found. Consider two representations $\mathbf{r}$ and $\mathbf{r}^{\prime}$ : We begin with the set $S_{1}$ of tensors in the product:

$$
S_{1} \equiv\left\{v_{1} \times v_{2} \mid v_{1} \in \mathbf{r}, v_{2} \in \mathbf{r}^{\prime}\right\}
$$

where the outer product of two tensors is defined in Section 3.1. However, we know that $S_{1}$ is not irreducible in itself, but instead spans some number of irreducible representations. The task then is to construct another set of tensors, $S_{2}$, which contains states of definite hypercharge and isospin in irreducible representations only, and which is spanned by $S_{1}$ :

$$
S_{2} \equiv\left\{v \in \mathbf{R} \mid \mathbf{R} \subset \operatorname{span}\left(S_{1}\right)\right\}
$$


The Clebsch-Gordan coefficients are then simply the inner product of tensors in $S_{1}$ with tensors in $S_{2}$ :

$$
\left\langle v_{2} \mid v_{1}\right\rangle=v_{2} \cdot v_{1} \quad\left(v_{1} \in S_{1}, v_{2} \in S_{2}\right) .
$$

The crux of the entire calculation is the decomposition of $S_{1}$ into irreducible representations. The method is the $S U(3)$ analogy to the method by which we decomposed states at the same hypercharge into $S U(2)$ multiplets in the previous section. We begin with the highest-weight tensor in $S_{1}$. There can be only one state with highest weight in $S_{1}$. From it we construct the "largest" representation in $S_{2}$, as described in Section 3.2. By "largest" we simply mean having a highestweight state with weight (quantum numbers $y$ and $i_{3}$ ) higher than those of the other representations. A state with quantum numbers $\left(y, i, i_{3}\right)$ has a higher weight than $\left(y^{\prime}, i^{\prime}, i_{3}^{\prime}\right)$ if either $y>y^{\prime}$ or both $y=y^{\prime}$ and $i_{3}>i_{3}^{\prime}$. Strangely, under this definition, the $\overline{\mathbf{2 1}}$ is "larger" than the $\overline{\mathbf{2 4}}$. Consider now the set of tensors remaining after the largest representation is constructed. Of them, we take the subset with the highest weight and project out the parts that are orthogonal to the states of the largest representation. Unfortunately, this set may or may not contain only one linearly independent tensor. This fact is called the "outer degeneracy problem." In such a case we must appeal to other considerations. We have adopted the convention of [37] in order to resolve outer degeneracies. The highest outer degeneracy in this work is two, and is only present when one of the factors is an octet. The prescription of [37] corresponds to constructing the representations in the product so that the highestisospin state of only one of them to couples to the isospin-1 multiplet in the factor octet. Once the highest-weight state(s) of the second-largest representation(s) is (are) found, the representation(s) is (are) constructed again as in Section 3.2.

It remains to specify the overall phases of representations in the decomposition of the product of two irreducible representations. We choose to follow the phase convention of de Swart [31]. For each representation $\mathbf{R}$ in the product, consider the state with highest third component of isospin and call its quantum numbers $Y_{\mathrm{h}}$, 
$I_{\mathrm{h}}$, and $I_{\mathrm{h} 3}$. Next consider the state in the first factor representation ( $\mathbf{r}$ ) that has highest isospin and couples to $\mathbf{R}\left(Y_{\mathrm{h}}, I_{\mathrm{h}}, I_{\mathrm{h} 3}\right)$; call its quantum numbers $y_{\mathrm{h}}, i_{\mathrm{h}}$, and $i_{\text {h3 }}$. Now consider the state in the second factor representation $\left(r^{\prime}\right)$ with highest isospin that couples the above two states; its quantum numbers are labelled $y_{\mathrm{h}}^{\prime}, i_{\mathrm{h}}^{\prime}$, and $i_{\mathrm{h} 3}^{\prime}$. The phase convention requires that the Clebsch-Gordan coefficient between these three states be positive (and real):

$$
\left\langle\mathbf{R} Y_{\mathrm{h}} I_{\mathrm{h}} I_{\mathrm{h} 3} \mid \mathbf{r} y_{\mathrm{h}} i_{\mathrm{h}} i_{\mathrm{h} 3} \mathbf{r}^{\prime} y_{\mathrm{h}}^{\prime} i_{\mathrm{h}}^{\prime} i_{\mathrm{h} 3}^{\prime}\right\rangle>0 .
$$

With these phase conventions, the Clebsch-Gordan coefficients and isoscalar factors are real.

We continue this process until the span of $S_{1}$ has been completely decomposed into irreducible representations, and the sizes of $S_{1}$ and $S_{2}$ are equal. Then are we ready to calculate the Clebsch-Gordan coefficients. A more transparent way of defining the coefficients is

$$
\left\langle\mathbf{R} Y I I_{3} \mid \mathbf{r} y i i_{3} \mathbf{r}^{\prime} y^{\prime} i^{\prime} i_{3}^{\prime}\right\rangle=\mathbf{R}\left(Y, I, I_{3}\right) \cdot\left[\mathbf{r}\left(y, i, i_{3}\right) \times \mathbf{r}^{\prime}\left(y^{\prime}, i^{\prime}, i_{3}^{\prime}\right)\right]
$$

The resulting table of coefficients is a necessarily unitary matrix, and can be divided into unitary submatrices for each $\left(Y, I_{3}\right)$ pair. Moreover, our calculational method inherently gives a real matrix. The general case is a unitary, complex matrix. We check that every row and column has unit norm, and make spotchecks that rows and columns are orthogonal to one another.

\subsection{Isoscalar Factors}

Because there are two quantum numbers in $S U(3)$ weights, compared to one for $S U(2)$, the tables of $S U(3)$ Clebsch-Gordan coefficients take far more paper to display than do those of $S U(2)$. Fortunately, there exists a way to compress the information contained in such a table. Because the representations of $S U(3)$ can be viewed as collections of $S U(2)$ representations at different hypercharges, we 
can "factor out" the $S U(2)$ Clebsch-Gordan coefficients from those of $S U(3)$. The remaining factors are called the isoscalar factors. They are what appear in the tables at the end of this chapter.

The isoscalar factors depend of the identity of the representations, and on the hypercharges and isospins of the isomultiplets that are coupled. We will denote them by $F\left(\mathbf{R}, Y, I ; \mathbf{r}, y, i, \mathbf{r}^{\prime}, y^{\prime}, i^{\prime}\right)$. The $S U(3)$ Clebsch-Gordan coefficients are found as products of isoscalar factors and $S U(2)$ Clebsch-Gordan coefficients:

$\left\langle\mathbf{R} Y I I_{3} \mid \mathbf{r} y i i_{3} \mathbf{r}^{\prime} y^{\prime} i^{\prime} i_{3}^{\prime}\right\rangle=F\left(\mathbf{R}, Y, I ; \mathbf{r}, y, i, \mathbf{r}^{\prime}, y^{\prime}, i^{\prime}\right) \times\left\langle I I_{3} \mid i i_{3} i^{\prime} i_{3}^{\prime}\right\rangle$.

The $S U(2)$ tables can be reconstructed from Tables $1^{3}, 2^{3}, 3^{3}$, and $4^{3}$ of [42]. For isospin less than or equal to two, they can conveniently be found in the Review of Particle Properties [10]. Note the easily overlooked relation

$$
\left\langle I I_{3} \mid i i_{3} i^{\prime} i_{3}^{\prime}\right\rangle=(-1)^{I-i-i^{\prime}}\left\langle I I_{3} \mid i^{\prime} i_{3}^{\prime} i i_{3}\right\rangle \text {. }
$$

Note that for each pair of $Y$ and $I$ the table necessarily contains a unitary submatrix.

There are two symmetry relations among the isoscalar factors that will allow us to omit many tables from our exposition. Those tables can be reconstructed from those that are present, with the help of the phase factors involved in these symmetry relations. Both relations come from [31], but we rewrite them in our notation. ${ }^{3}$ The first involves the order of the factor representations. If the order is reversed, then a phase $\xi$ may enter:

$$
F\left(\mathbf{R}, Y, I ; \mathbf{r}^{\prime}, y^{\prime}, i^{\prime}, \mathbf{r}, y, i\right)=(-1)^{I-i-i^{\prime}} \xi\left(\mathbf{R} ; \mathbf{r}, \mathbf{r}^{\prime}\right) F\left(\mathbf{R}, Y, I ; \mathbf{r}, y, i, \mathbf{r}^{\prime}, y^{\prime}, i^{\prime}\right)
$$

The factor $(-1)^{I-i-i^{\prime}}$ comes from Equation 3.20. The phase $\xi\left(\mathbf{R} ; \mathbf{r}, \mathbf{r}^{\prime}\right)$ does not depend on the quantum numbers of the states, but only on the identity of the representations $\mathbf{r}, \mathbf{r}^{\prime}$, and $\mathbf{R}$, and on the phase conventions described in the previous section. The second symmetry relation involves the conjugation of the representations:

$$
F\left(\overline{\mathbf{R}}, Y, I ; \overline{\mathbf{r}}, y, i, \overline{\mathbf{r}^{\prime}}, y^{\prime}, i^{\prime}\right)=(-1)^{I-i-i^{\prime}} \zeta\left(\mathbf{R} ; \mathbf{r}, \mathbf{r}^{\prime}\right) F\left(\mathbf{R},-Y, I ; \mathbf{r},-y, i, \mathbf{r}^{\prime},-y^{\prime}, i^{\prime}\right)
$$

\footnotetext{
${ }^{3} \operatorname{Our} \xi$ is the $\xi_{1}$ of $[31] ; \zeta$ is $\xi_{3}$ of [31].
} 
Here $\zeta\left(\mathbf{R} ; \mathbf{r}, \mathbf{r}^{\prime}\right)$ also does not depend on the quantum numbers of the states involved, but only on the identities of the representations and on our phase conventions. For these relations, bars on self-adjoint representations are irrelevant. It is easy to show from Equations 3.21 and 3.22 that

$$
\xi\left(\overline{\mathbf{R}} ; \overline{\mathbf{r}}, \overline{\mathbf{r}^{\prime}}\right)=\xi\left(\mathbf{R} ; \mathbf{r}, \mathbf{r}^{\prime}\right)
$$

Then it can be shown (using Equation 3.23) that

$$
\zeta\left(\mathbf{R} ; \mathbf{r}^{\prime}, \mathbf{r}\right)=\zeta\left(\mathbf{R} ; \mathbf{r}, \mathbf{r}^{\prime}\right)
$$

The $\xi$ and $\zeta$ needed to construct the omitted tables are presented in Tables 3.2 and 3.3. We should note that it is not necessary to construct the tables related by Equations 3.21 and 3.22 in order to find the phase factors. They are found by considering the highest-isospin states of the representations in the product. Suppose that such a state and the highest-isospin states coupling to it are described as in Section 3.3. Then from Equation 3.20 we find simply that

$$
\zeta\left(\mathbf{R} ; \mathbf{r}, \mathbf{r}^{\prime}\right)=(-1)^{I_{\mathbf{h}}-i_{\mathrm{h}}-i_{\mathrm{h}}^{\prime}}
$$

Suppose that in the reversed product $\mathbf{r}^{\prime} \otimes \mathbf{r}$ the highest-isospin state in $\mathbf{r}^{\prime}$ that couples to $I_{\mathbf{h}}$ in $\mathbf{R}$ has quantum numbers $y_{\mathrm{h}}^{\text {rev }}$ and $i_{\mathbf{h}}^{\text {rev }}$, and the highest isospin in $\mathbf{r}$ that couples to these two has $y_{\mathrm{h}}^{\mathrm{rev}}$ and $i_{\mathrm{h}}^{\text {rev }}$. Then

$$
\xi\left(\mathbf{R} ; \mathbf{r}, \mathbf{r}^{\prime}\right)=(-1)^{I_{\mathbf{h}}-i_{\mathrm{h}}^{\text {rev }}-i_{\mathrm{h}}^{\text {rev }}} \times \operatorname{sign}\left[F\left(\mathbf{R}, Y_{\mathrm{h}}, I_{\mathrm{h}} ; \mathbf{r}, y_{\mathrm{h}}^{\mathrm{rev}}, i_{\mathrm{h}}^{\mathrm{rev}}, \mathbf{r}^{\prime}, \dot{y}_{\mathrm{h}}^{\text {rev }}, i_{\mathrm{h}}^{\text {rev }}\right)\right]
$$

where $\operatorname{sign}(x)=x /|x|$.

\subsection{Computer Implementation}

The calculations of Clebsch-Gordan coefficients were performed on computer. The routines are described in this chapter. Many of them have also been described in [44]. The computers used were the VAX 4090 and VAX 6610, running VMS 
The programming language used was Pascal, and the program was compiled with DEC Pascal version 5.1. Because we take the approach of explicitly constructing the representations and use integers for exact precision, the main limitations of the routines are due to memory allocation and integer overflows. In some problem cases, we are able to complete a table by constructing it in pieces, or by inferring the coefficients of a missing representation by imposing unitarity on the table. For example, the singlet in the product $\overline{\mathbf{1 0}} \otimes \mathbf{1 0}$ cannot be constructed in the algorithm described in the previous section, since finding the tensor that is orthogonal to all larger representations in the product overflows the integers used. The coefficients involving the singlet are uniquely determined, up to an overall phase, by unitarity of the submatrix at $y=0, i_{3}=0$, and so can be inferred. The phase is determined by our conventions.

Unlike earlier published programs to generate $S U(3)$ Clebsch-Gordan coefficients [36] [37] [38], we do not use floating-point variables, but instead calculate the coefficients exactly with integer variables. A rational variable type (called "fraction") is defined for this purpose. Also to this end, states of representations are stored as arrays of integers (called "vectors"). It is possible to represent states of $S U(3)$ representations as tensors with rational coefficients due to the finite nature of the group. That is, the fact that the quantum numbers of $S U(3)$ are discrete enables all calculations to be done with rational numbers. The table of Clebsch-Gordan coefficients is then stored in an array of fractions, each of which is the signed square of the corresponding coefficient.

The remainder of this section is the description of the most important variables and routines in the program. They are divided into those that deal with tensors, those that deal with representations, those that deal with the process of tabulating the coefficients, and those that decompose the set $S_{1}$ (see Section 3.3) into $S_{2}$ (these are the routines do $\mathrm{RxS}$ ). 


\subsubsection{Tensors}

The vector $n$ variable type is an array of $3^{n}+1$ integers, where the +1 is for space allocated for the norm squared. The norm serves as a common denominator for the fractions that are the components of a tensor. A different type is defined for each $n$, up to a limit of nine. What are the implications of this storage method? Two main difficulties arise. The first is that we need a different routine for each possible number of indices of a tensor. These routines are distinguished in the following descriptions by the suffix " $n$." This makes it difficult to develop a general routine to tabulate the Clebsch-Gordan coefficients of representations using arbitrary numbers of indices. The second is the limitation of computer memory. We cannot compile the table of coefficients for the product of two arbitrary representations, but must cease when the representations are too large.

There are routines designed to return certain information about state tensors. The function nulln returns true if a tensor is the zero tensor. The routines hyperchargen, totisospin $n$, and isospin $n$ return the quantum numbers $y, i$, and $i_{3}$ of a state. Testing whether a state is of highest weight is done by checkhighest $n$, which uses $T_{-} n$ (described below). These routines are used throughout the program.

Various manipulations of state tensors are needed. The inner and outer products of tensors are found with innern and outer $m \times n$. Multiplication and division is used in finding orthogonal projections of states and for normalizing states; for this scalardivide $n$ and scalarmult $n$ are called. The normalizing routine is normalize $n$, while findperp $n$ projects one state onto the subspace orthogonal to another state. Finally, the ladder operations are performed by the routines $T_{-} n$. The direction of the operation is passed as an argument, so that one routine can perform all six ladder operations. 


\subsubsection{Representations}

The representations are stored in a variable type called rep_n. It is merely a collection of vector variables, one for each state in the representation. The routines which fill these variables are called calc $\mathbf{R} \_n$, for each representation $\mathbf{R}$ and dimension of tensors $n$. These routines begin with the highest-weight state and from it call the ladder operation routines in order to find the remaining states. In the cases of multiple degeneracy in $\left(y, i_{3}\right)$, findperp $n$ is used in order to resolve the states into those of definite total isospin $i$. This is discussed in Section 3.2 .

\subsubsection{The Table of Coefficients}

The table variable is simply an array of fraction. Each entry is the signed square of its corresponding Clebsch-Gordan coefficient. Entries are placed in table by tabulate $m \times n$, which calls the routines that take the appropriate inner and outer products in order to implement Equation 3.18. The table is then checked for normality in both directions by normalhorizontal and normalvertical. Finally, the table is written in $\operatorname{LT}_{\mathrm{E}} \mathrm{X}$ format by latextable. This makes life easier for us humans.

\subsubsection{The Tensor Decomposition}

The routines that handle the decomposition of the set $S_{1}$ into the set $S_{2}$ are called doRxS, where $\mathbf{R}$ and $\mathbf{S}$ denote the names of the factor representations. The method employed is that described in Section 3.3. These routines call calcR_n to generate representations, and findperp $n$ to find the highest-weight states of smaller representations. For each representation in the product, a call to tabulatemx $n$ fills the corresponding columns in the table. After the table of coefficients is complete, normalhorizontal and normal vertical are called to verify normality, latextable is used to place the coefficients into the output file, and isoscalar (described be- 
low) is called to extract and record the isoscalar factors.

\subsubsection{Isoscalar Factor Extraction}

A routine called iscoscalar is used to extract the isoscalar factors from the table of Clebsch-Gordan coefficients. It does this by compiling the necessary tables of $S U(2)$ Clebsch-Gordan coefficients and dividing them into the $S U(3)$ Clebsches. Although we need only use one Clebsch from each isomultiplet, as a check we consider each member of the isomultiplet and verify that each gives the same isoscalar factor. The isoscalar factors are at last written in $\mathrm{IAT}_{\mathrm{E}} \mathrm{X}$ format to a file for our use.

\subsection{The Tables of Isoscalar Factors}

Here are presented the tables of isoscalar factors for $S U(3)$. Recovering the ClebschGordan coefficients is accomplished with Equation 3.19. The list of tables appears in Table 3.1 .

The tables follow. Table 3.1 is a list of the products of representations whose isoscalar factors are given in this work. Tables 3.2 and 3.3 give the phase factors which are described in the previous section and which can be used to generate other tables from the ones presented. The tables of isoscalar factors appear last. Tables for $8 \otimes 8,10 \otimes 8,10 \otimes 10, \overline{10} \otimes 10$, and $27 \otimes 8$ also appear in [31] and [32]. Only the tables needed for the remainder of this work are presented here. The interested reader can find additional tables of isoscalar factors in [45]. In the tables, a square root is assumed to appear over each entry (signs are outside the square roots). Thus,

\begin{tabular}{cccc|c} 
& & & & $\mathbf{R}$ \\
& $\mathbf{r} \otimes \mathbf{r}^{\prime}$ & $Y$ \\
& & & $I$ \\
\hline$y$ & $i$ & $y^{\prime}$ & $i^{\prime}$ & $\pm C$ \\
\hline
\end{tabular}


means that the isoscalar factor

$$
F\left(\mathbf{R}, Y, I ; \mathbf{r}, y, i, \mathbf{r}^{\prime}, y^{\prime}, i^{\prime}\right)= \pm \sqrt{C}
$$


Table 3.1: List of tables of isoscalar factors that follow.

Table $3.4 \quad \mathbf{3} \otimes \mathbf{3}=\mathbf{6} \oplus \overline{\mathbf{3}}$

Table $\quad 3.5 \quad \overline{3} \otimes 3=8 \oplus 1$

Table $3.6 \quad 6 \otimes 3=10 \oplus 8$

Table $3.7 \quad 6 \otimes \overline{3}=15 \oplus 3$

Table $3.8 \quad 8 \otimes \mathbf{3}=\mathbf{1 5} \oplus \overline{\mathbf{6}} \oplus \mathbf{3}$

Table $\quad 3.9 \quad 8 \otimes 8=27 \oplus \overline{\mathbf{1 0}} \oplus \mathbf{8} 10 \oplus \mathbf{8}_{1} \oplus \mathbf{8}_{2} \oplus \mathbf{1}$

Table $3.1010 \otimes 8=35 \oplus 27 \oplus 10 \oplus 8$

Table $3.11 \overline{10} \otimes 10=64 \oplus 27 \oplus 8 \oplus 1$

Table $3.1215 \otimes \overline{3}=27 \oplus \mathbf{1 0} \oplus 8$

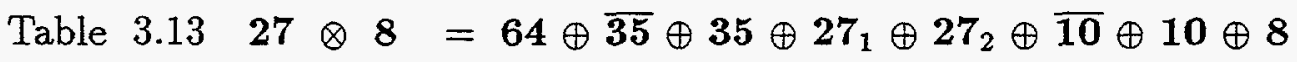

Table $3.14 \quad 27 \otimes 27 \quad$ (to singlet only) 
Table 3.2: $\quad$ Phase factors $\xi$. Here $(+)$ denotes +1 and $(-)$ denotes -1 .

\begin{tabular}{|c|c|c|c|c|c|c|c|c|c|c|c|c|c|c|}
\hline \multicolumn{2}{|c|}{$\xi$} & \multicolumn{13}{|c|}{$\mathbf{R}$} \\
\hline $\mathbf{r}$ & $\mathbf{r}^{\prime}$ & 13 & $3 \overline{3}$ & 6 & $\overline{6} 8$ & $8_{2}$ & 10 & $\overline{\overline{10}}$ & 15 & $27_{1}$ & $27_{2}$ & 35 & $\overline{\mathbf{3 5}}$ & 64 \\
\hline 3 & 3 & & - & + & & & & & & & & & & \\
\hline$\overline{3}$ & 3 & - & & & + & & & & & & & & & \\
\hline 6 & 3 & & & & - & & + & & & & & & & \\
\hline 6 & $\overline{\mathbf{3}}$ & - & & & & & & & + & & & & & \\
\hline 8 & 3 & - & & & - & & & & + & & & & & \\
\hline 8 & 8 & + & & & + & - & - & - & & + & & & & \\
\hline 10 & 8 & & & & + & & - & & & - & & + & & \\
\hline$\overline{\mathbf{1 0}}$ & 10 & - & & & + & & & & & - & & & & + \\
\hline 15 & $\overline{\mathbf{3}}$ & & & & - & & - & & & + & & & & \\
\hline 27 & 8 & & & & + & & + & + & & - & + & - & - & + \\
\hline
\end{tabular}

Table 3.3: $\quad$ Phase factors $\zeta$. Here $(+)$ denotes +1 and $(-)$ denotes -1 .

\begin{tabular}{|c|c|c|c|c|c|c|c|c|c|c|c|c|c|c|c|}
\hline \multicolumn{2}{|c|}{$\zeta$} & \multicolumn{14}{|c|}{$\mathbf{R}$} \\
\hline $\mathbf{r}$ & $\mathbf{r}^{\prime}$ & 1 & 3 & $\overline{\overline{\mathbf{3}}}$ & $6 \overline{6}$ & $8_{1}$ & $\overline{8_{2}}$ & 10 & $\overline{\mathbf{1 0}}$ & 15 & $27_{1}$ & $27_{2}$ & 35 & $\overline{\mathbf{3 5}}$ & 64 \\
\hline 3 & 3 & & & + & $t$ & & & & & & & & & & \\
\hline$\overline{3}$ & 3 & - & & & & + & & & & & & & & & \\
\hline 6 & 3 & & & & & + & & + & & & & & & & \\
\hline 6 & $\overline{\mathbf{3}}$ & & - & & & & & & & + & & & & & \\
\hline 8 & 3 & & - & & + & & & & & + & & & & & \\
\hline 8 & 8 & + & & & & + & - & + & + & & + & & & & \\
\hline 10 & 8 & & & & & - & & - & & & + & & + & & \\
\hline$\overline{\mathbf{1 0}}$ & 10 & - & & & & + & & & & & - & & & & + \\
\hline 15 & $\overline{3}$ & & & & & - & & + & & & + & & & & \\
\hline 27 & 8 & & & & & + & & - & - & & - & + & + & + & + \\
\hline
\end{tabular}


Table 3.4: $\quad$ Isoscalar factors for $\mathbf{3} \otimes \mathbf{3}$. Notation for this and following tables is explained in the text. In these tables a square root is assumed over each entry.

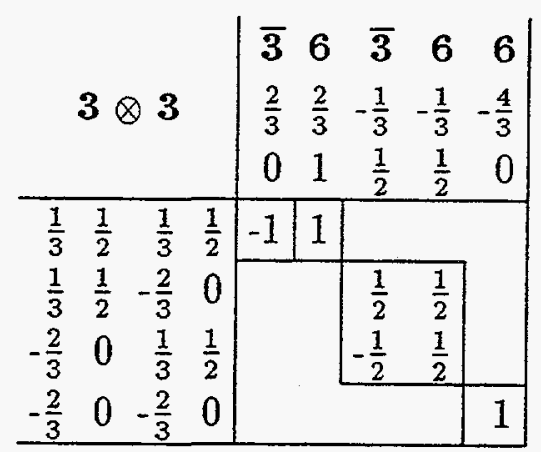

Table 3.5: $\quad$ Isoscalar factors for $\overline{\mathbf{3}} \otimes \mathbf{3}$.

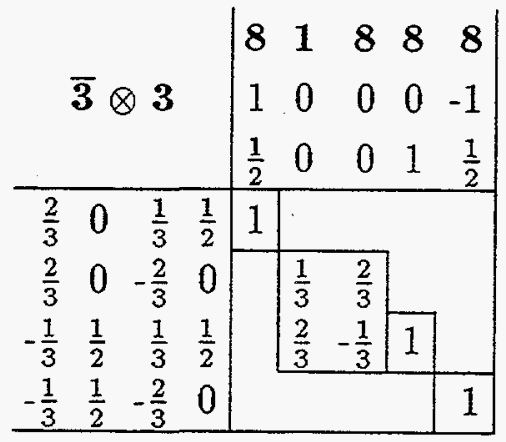

Table 3.6: $\quad$ Isoscalar factors for $\mathbf{6} \otimes \mathbf{3}$.

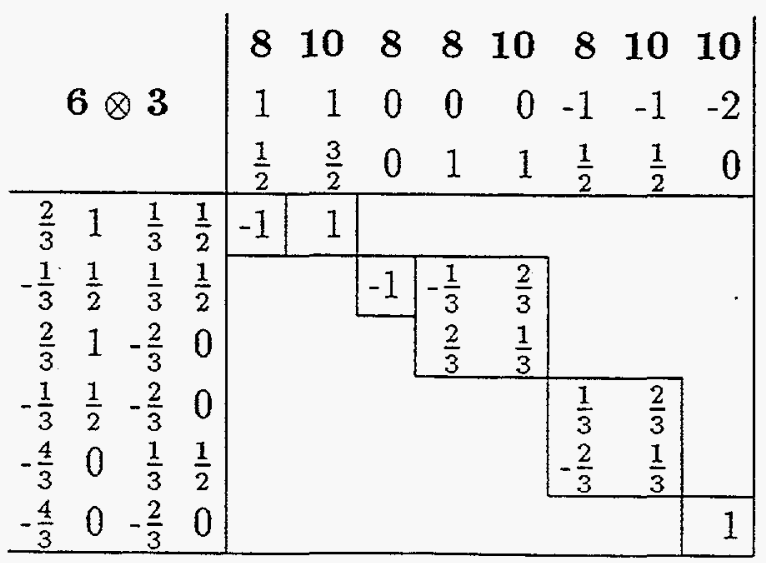


Table 3.7: $\quad$ Isoscalar factors for $\mathbf{6} \otimes \overline{\mathbf{3}}$.

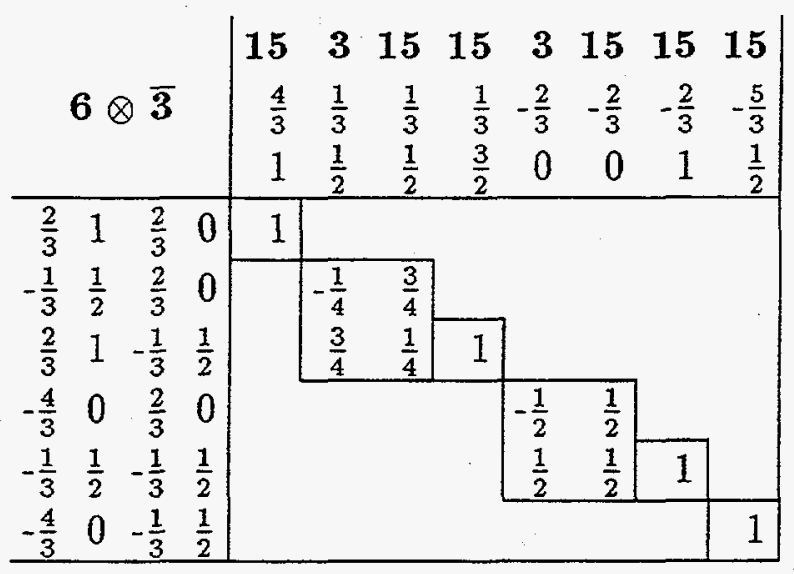

Table 3.8: $\quad$ Isoscalar factors for $\mathbf{8} \otimes \mathbf{3}$.

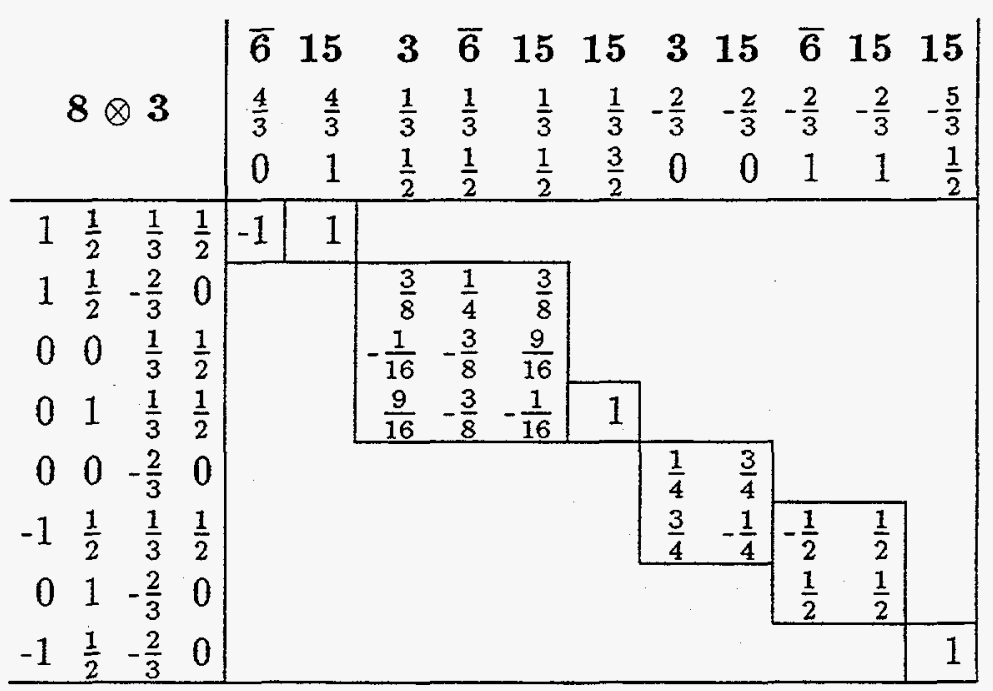


Table 3.9: $\quad$ Isoscalar factors for $8 \otimes \mathbf{8}$.

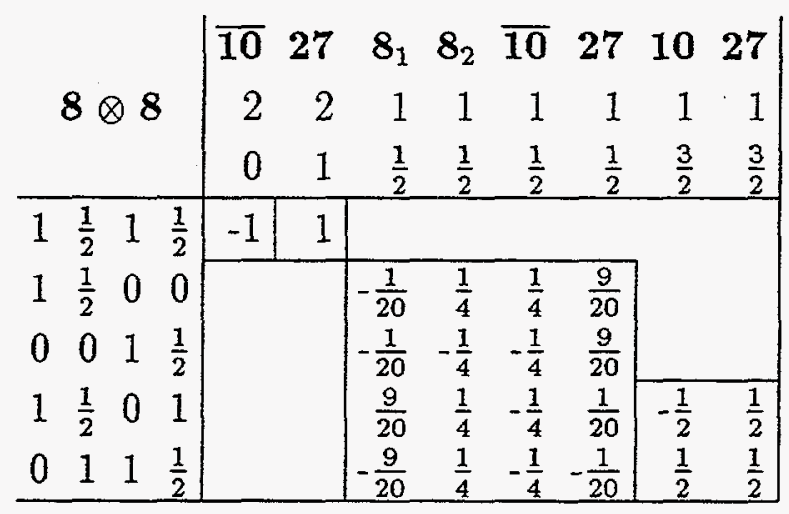

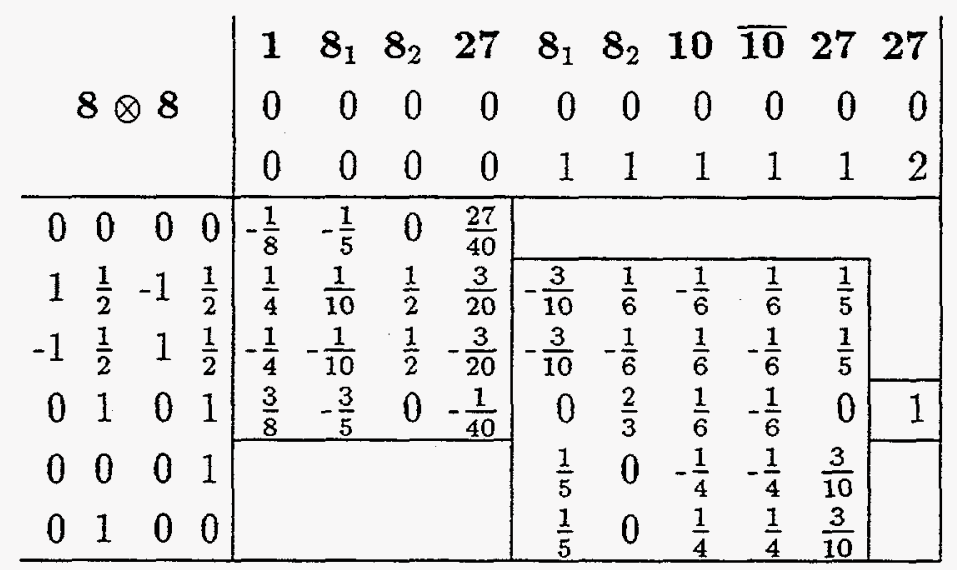

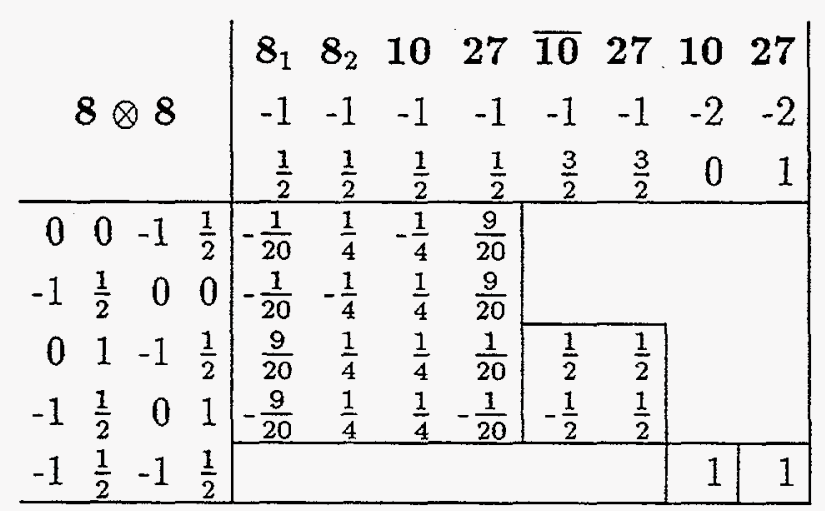


Table 3.10: $\quad$ Isoscalar factors for $\mathbf{1 0} \otimes \mathbf{8}$.

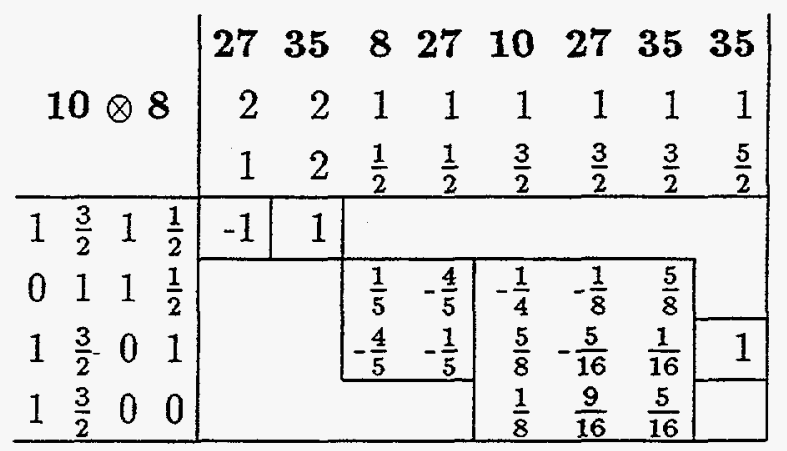

\begin{tabular}{|c|c|c|c|c|c|c|c|}
\hline \multirow{3}{*}{$10 \otimes 8$} & 827 & 8 & 10 & 27 & 35 & & 35 \\
\hline & & 0 & 0 & 0 & 0 & 0 & \\
\hline & $\begin{array}{ll}0 & 0\end{array}$ & 1 & 1 & 1 & 1 & 2 & \\
\hline $\begin{array}{llll}-1 & \frac{1}{2} & 1 & \frac{1}{2}\end{array}$ & $\begin{array}{|ll|}\frac{2}{5} & -\frac{3}{5} \\
\end{array}$ & $\frac{2}{15}$ & $-\frac{1}{3}$ & $-\frac{1}{5}$ & $\frac{1}{3}$ & & \\
\hline $\begin{array}{llll}0 & 1 & 0 & 1\end{array}$ & $\left|\begin{array}{ll}-\frac{3}{5} & -\frac{2}{5}\end{array}\right|$ & $-\frac{2}{15}$ & $\frac{1}{3}$ & & $\frac{1}{12}$ & & \\
\hline $1 \frac{3}{2}-1 \quad \frac{1}{2}$ & & $\frac{8}{15}$ & $\frac{1}{3}$ & $\frac{1}{20}$ & $\frac{1}{12}$ & & \\
\hline $\begin{array}{llll}0 & 1 & 0 & 0\end{array}$ & & $-\frac{1}{5}$ & 0 & $\frac{3}{10}$ & $\frac{1}{2}$ & & \\
\hline
\end{tabular}

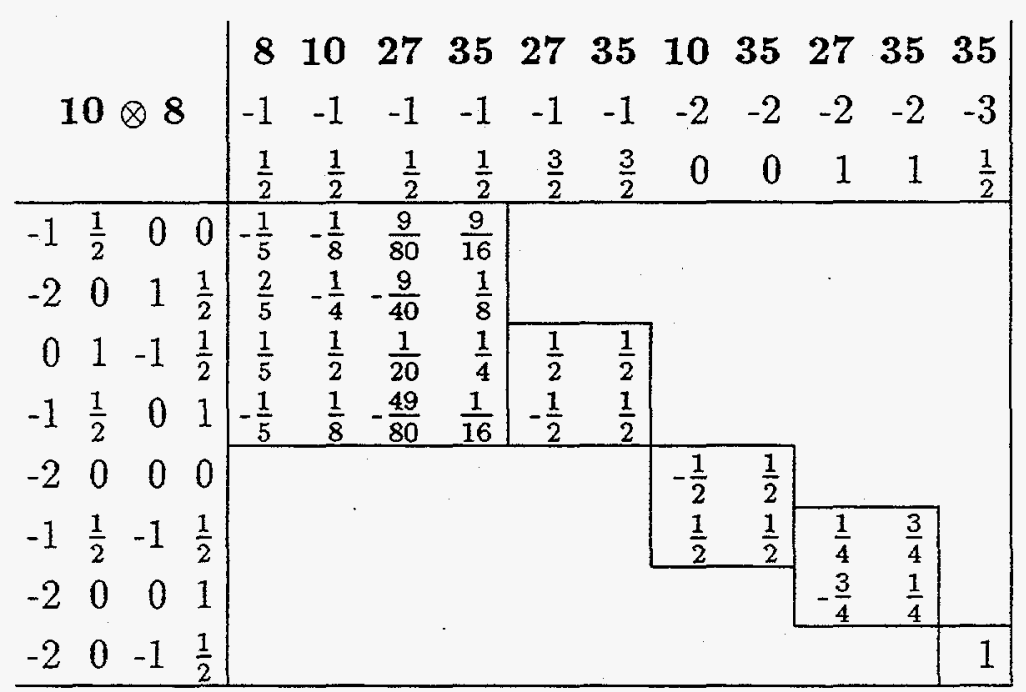


Table 3.11: $\quad$ Isoscalar factors for $\overline{\mathbf{1 0}} \otimes \mathbf{1 0}$.

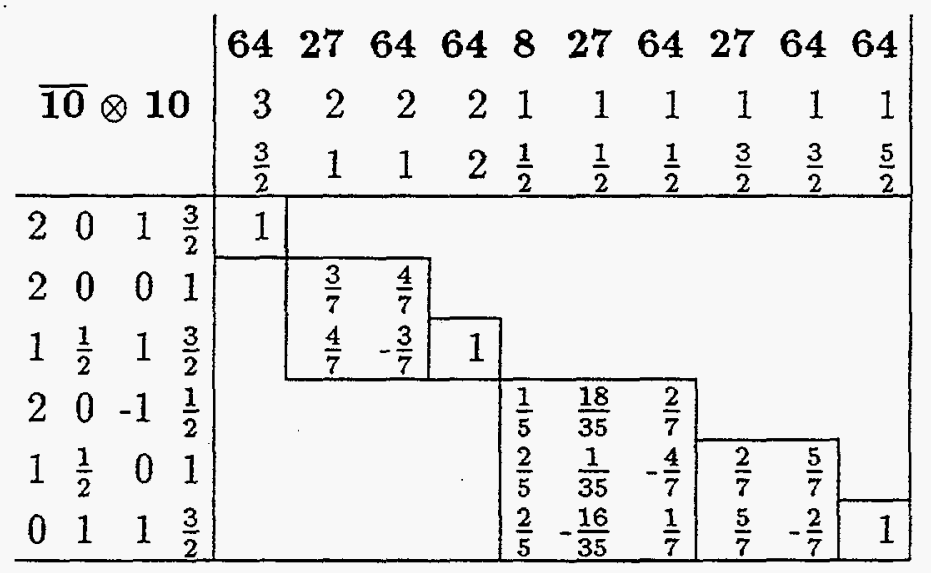

\begin{tabular}{|c|c|c|c|c|c|c|c|c|c|c|}
\hline \multirow{2}{*}{$\overline{10} \otimes 10$} & $\begin{array}{l}1 \\
0\end{array}$ & $\begin{array}{l}8 \\
0\end{array}$ & $\begin{array}{r}27 \\
0\end{array}$ & $\begin{array}{r}84 \\
0\end{array}$ & 0 & 0 & 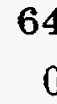 & 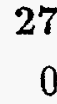 & 0 & 0 \\
\hline & 0 & 0 & 0 & 0 & 1 & 1 & 1 & 2 & 2 & 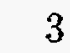 \\
\hline $\begin{array}{lllll}2 & 0 & -2 & 0\end{array}$ & $\frac{1}{10}$ & $\frac{2}{5}$ & $\frac{27}{70}$ & $\overline{3}$ & & & & & & \\
\hline $1 \frac{1}{2}-1 \frac{1}{2}$ & $\frac{1}{5}$ & & & $-\frac{18}{35}$ & & & $\frac{10}{21}$ & & & \\
\hline $\begin{array}{llll}0 & 1 & 0 & 1\end{array}$ & & 0 & $-\frac{5}{14}$ & $\frac{12}{35}$ & $\frac{4}{15}$ & $\frac{9}{35}$ & $-\frac{10}{21}$ & & $\frac{6}{7}$ & \\
\hline $\begin{array}{llll}-1 & \frac{3}{2} & 1 & \frac{3}{2}\end{array}$ & $\frac{2}{5}$ & & $\frac{6}{35}$ & $-\frac{1}{35}$ & 2 & $-\frac{2}{7}$ & 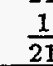 & & $-\frac{1}{7}$ & 1 \\
\hline
\end{tabular}

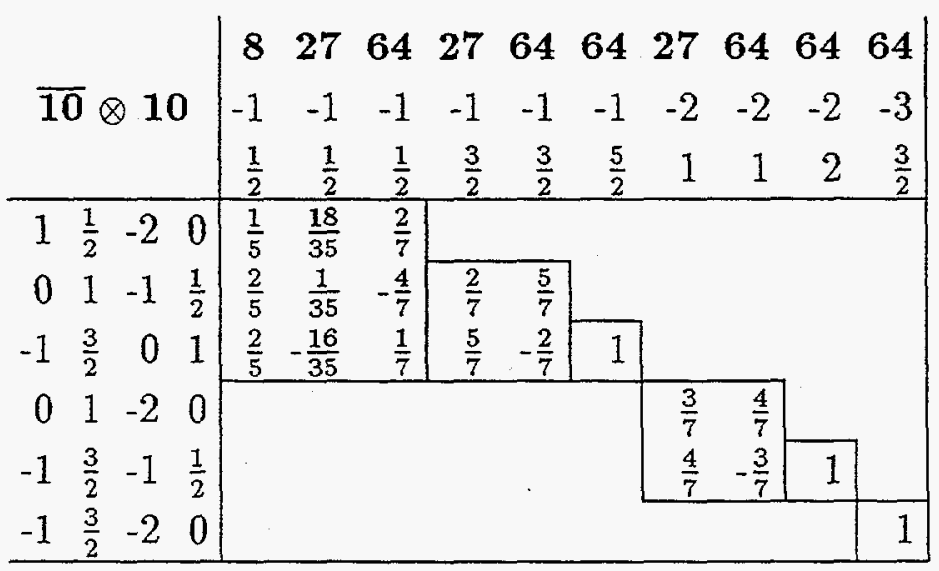


Table 3.12: $\quad$ Isoscalar factors for $\mathbf{1 5} \otimes \overline{\mathbf{3}}$.

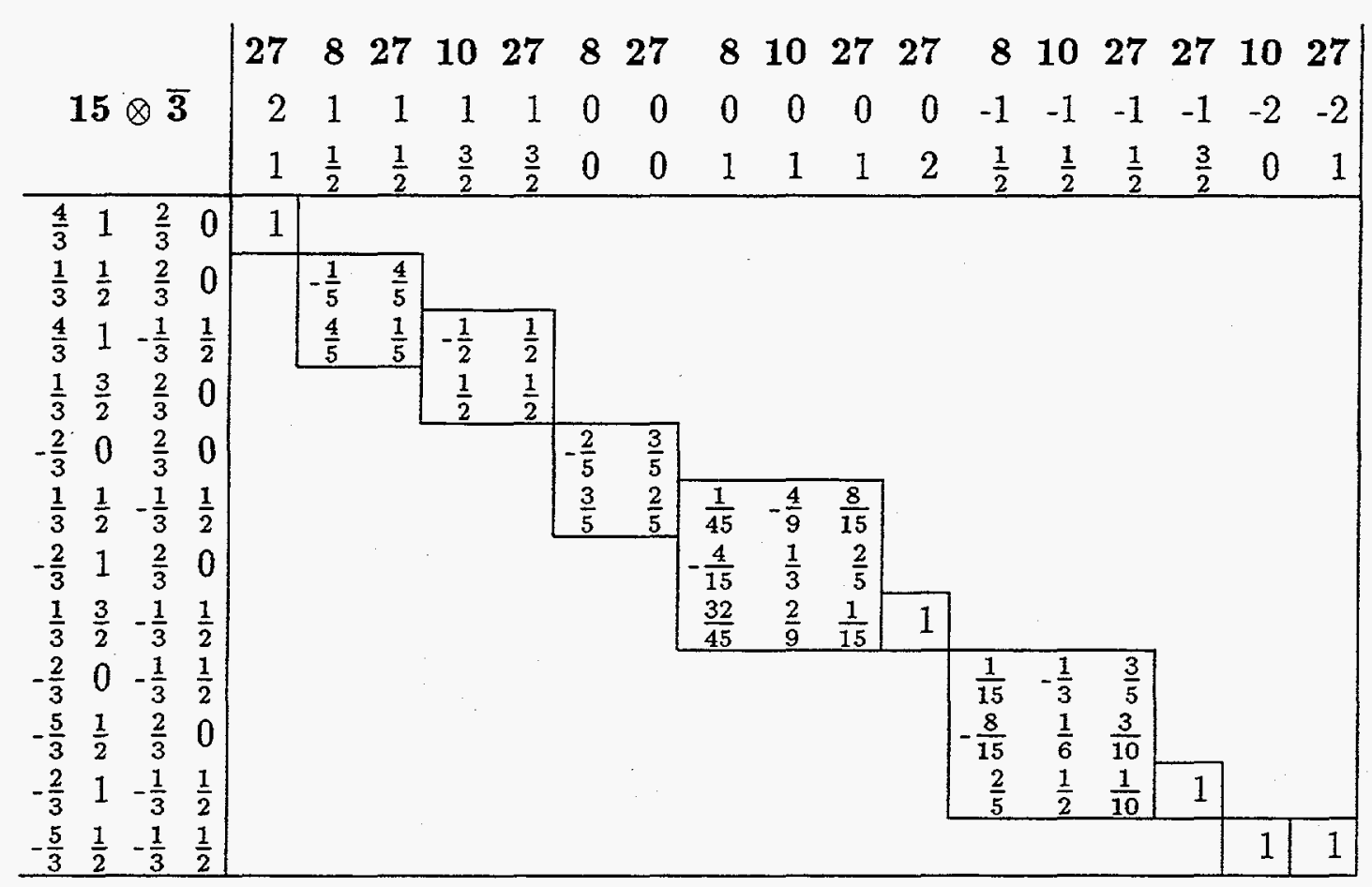


Table 3.13: $\quad$ Isoscalar factors for $\mathbf{2 7} \otimes \mathbf{8}$.

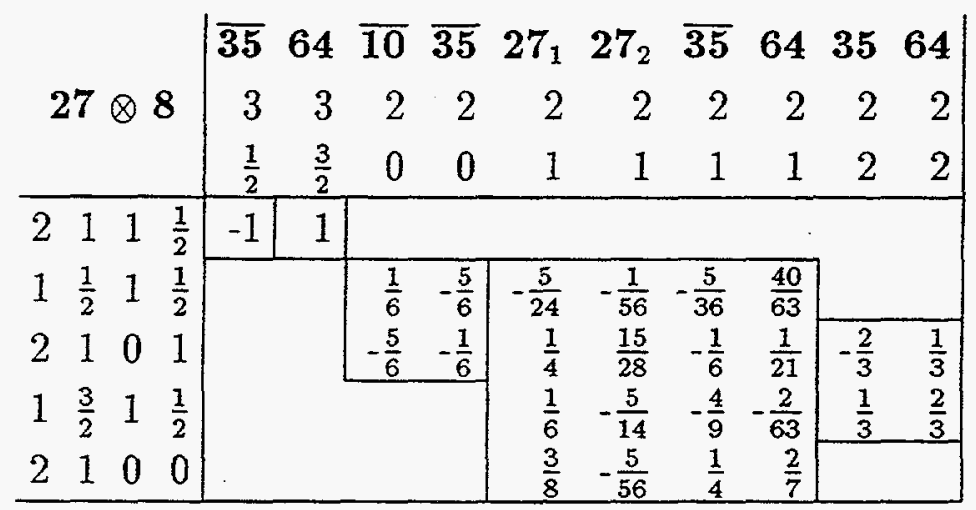

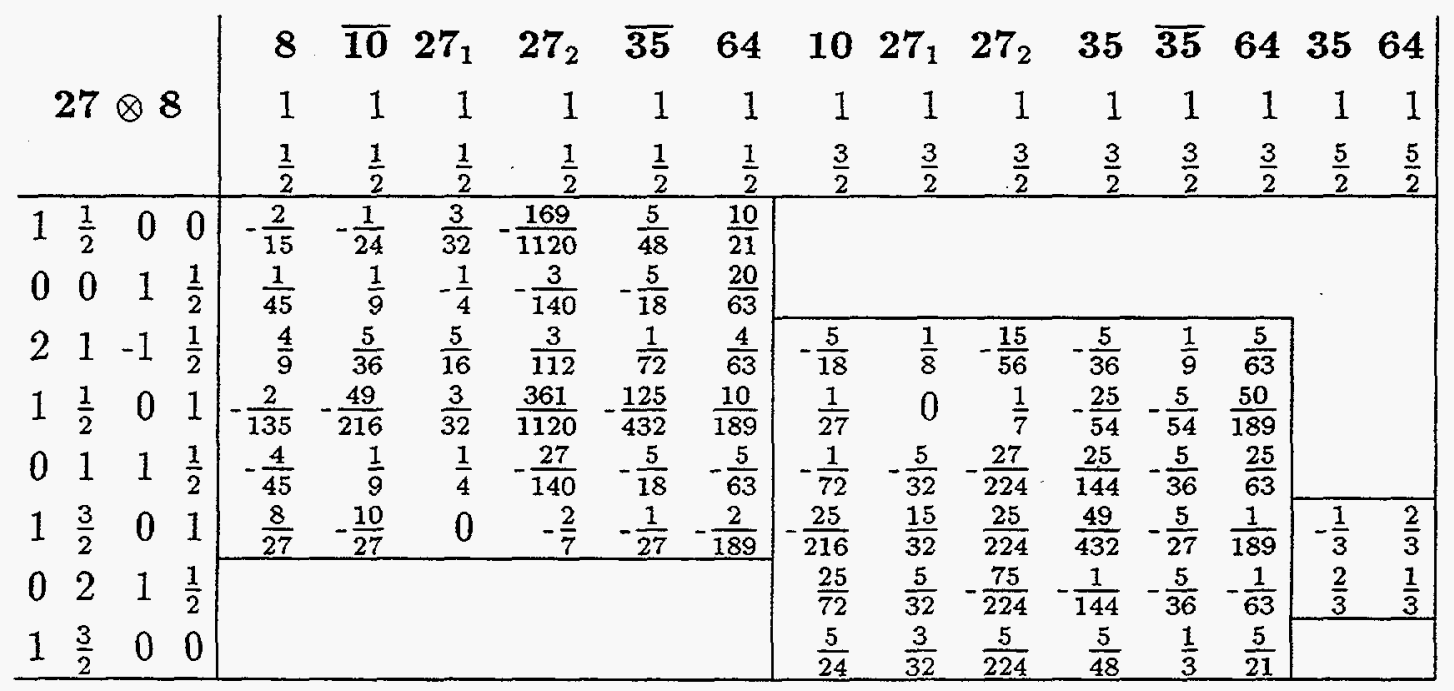

\begin{tabular}{|c|c|c|c|c|c|c|c|c|c|c|c|c|c|}
\hline \multirow{2}{*}{\multicolumn{2}{|c|}{$27 \otimes 8$}} & 8 & $27_{1}$ & $27_{2}$ & 64 & 8 & 10 & $\overline{10}$ & $27_{1}$ & $27_{2}$ & 35 & $\overline{35}$ & 64 \\
\hline & & 0 & 0 & 0 & 0 & 0 & 0 & 0 & 0 & 0 & 0 & 0 & 0 \\
\hline & & & & & & & & & & & & & \\
\hline 0 & $\begin{array}{lll}0 & 0\end{array}$ & $-\frac{1}{5}$ & 0 & $-\frac{8}{35}$ & & & & & & & & & \\
\hline 1 & $\frac{1}{2}-1$ & $\frac{4}{15}$ & $\frac{1}{2}$ & $\frac{3}{70}$ & $\frac{4}{21}$ & & & $\frac{1}{81}$ & $\frac{1}{6}$ & $-\frac{9}{70}$ & $-\frac{20}{81}$ & 81 & $\frac{100}{567}$ \\
\hline-1 & $\frac{1}{2} \quad 1$ & $-\frac{4}{15}$ & $\frac{1}{2}$ & $-\frac{3}{70}-$ & $-\frac{4}{21}$ & $\frac{4}{405}$ & $-\frac{1}{81}$ & $\frac{16}{81}$ & $-\frac{1}{6}$ & $-\frac{9}{70}$ & $\frac{5}{81}$ & $\frac{20}{81}$ & 567 \\
\hline 0 & 10 & $\frac{4}{15}$ & 0 & $-\frac{24}{35}-$ & $-\frac{1}{21}$ & 0 & $-\frac{1}{6}$ & $-\frac{1}{6}$ & $\frac{1}{4}$ & 0 & $\frac{5}{24}$ & $\frac{5}{24}$ & 0 \\
\hline & 10 & & & & & & $\frac{1}{9}$ & $-\frac{1}{9}$ & 0 & $\frac{1}{70}$ & $\frac{5}{36}$ & $\frac{\pi}{36}$ & $\frac{25}{63}$ \\
\hline & $\begin{array}{ll}0 & 0\end{array}$ & & & & & $\frac{1}{405}$ & $\frac{4}{81}$ & $-\frac{4}{81}$ & 0 & $\frac{8}{35}$ & $-\frac{20}{81}$ & $-\frac{20}{81}$ & $\frac{100}{567}$ \\
\hline 1 & $\frac{3}{2}-1$ & & & & & $\frac{16}{81}$ & $\frac{5}{81}$ & $\frac{20}{81}$ & $\frac{5}{24}$ & $\frac{9}{56}$ & $\frac{25}{324}$ & $\frac{1}{81}$ & $\frac{20}{567}$ \\
\hline-1 & $\frac{3}{2} \quad 1$ & & & & & $-\frac{16}{81}$ & $\frac{20}{81}$ & $\frac{5}{81}$ & $\frac{5}{24}$ & $-\frac{9}{56}$ & $-\frac{1}{81}$ & $-\frac{25}{324}$ & $-\frac{20}{567}$ \\
\hline 0 & $\begin{array}{lll}2 & 0\end{array}$ & & & & & $\frac{40}{81}$ & $\frac{25}{162}$ & $-\frac{25}{162}$ & 0 & $\overline{28}$ & $\overline{648}$ & $\frac{5}{648}$ & 56 \\
\hline
\end{tabular}




\begin{tabular}{rrrr|rrrrrr|}
27 & $\otimes$ & 8 & & $27_{1}$ & $27_{2}$ & 35 & $\overline{35}$ & 64 & 64 \\
0 & 0 & 0 & 0 & 0 & 0 \\
\hline 0 & 1 & 0 & 1 & 0 & $\frac{3}{28}$ & $-\frac{5}{24}$ & $-\frac{5}{24}$ & $\frac{10}{21}$ & \\
1 & $\frac{3}{2}$ & -1 & $\frac{1}{2}$ & $\frac{1}{8}$ & $-\frac{15}{56}$ & $-\frac{1}{12}$ & $\frac{1}{3}$ & $\frac{4}{21}$ & \\
-1 & $\frac{3}{2}$ & 1 & $\frac{1}{2}$ & $-\frac{1}{8}$ & $-\frac{15}{56}$ & $\frac{1}{3}$ & $-\frac{1}{12}$ & $\frac{4}{21}$ & \\
0 & 2 & 0 & 0 & 0 & $\frac{5}{14}$ & $\frac{1}{4}$ & $\frac{1}{4}$ & $\frac{1}{7}$ & \\
0 & 2 & 0 & 1 & $\frac{3}{4}$ & 0 & $\frac{1}{8}$ & $-\frac{1}{8}$ & 0 & 1 \\
\hline
\end{tabular}

\begin{tabular}{|c|c|c|c|c|c|c|c|c|c|c|c|c|c|}
\hline & 8 & 10 & $27_{1}$ & $27_{2}$ & 35 & 64 & 10 & $27_{1}$ & $\mathbf{2 7} \mathbf{7}_{2}$ & 35 & $\overline{35}$ & 64 & $\overline{35}$ \\
\hline $27 \otimes 8$ & -1 & -1 & -1 & -1 & -1 & -1 & -1 & -1 & -1 & -1 & -1 & -1 & -1 \\
\hline & & & & & & & & & & & & & \\
\hline $\begin{array}{llll} & \frac{1}{2} & 0 & 0\end{array}$ & $-\frac{2}{15}$ & $\frac{1}{24}$ & $-\frac{3}{32}$ & $-\frac{169}{1120}$ & $\frac{5}{48}$ & $\frac{10}{21}$ & & & & & & & \\
\hline $\begin{array}{llll}0 & 0 & -1 & \frac{1}{2}\end{array}$ & $\frac{1}{45}$ & $-\frac{1}{9}$ & $\frac{1}{4}$ & $-\frac{3}{140}$ & $-\frac{5}{18}$ & & & & & & & & \\
\hline $\begin{array}{llll}-2 & 1 & 1 & \frac{1}{2}\end{array}$ & $-\frac{4}{9}$ & $\frac{5}{36}$ & $\frac{5}{16}$ & $-\frac{3}{112}$ & $-\frac{1}{72}$ & $-\frac{4}{63}$ & $\frac{5}{18}$ & $-\frac{1}{8}$ & $-\frac{15}{56}$ & $\frac{1}{9}$ & $-\frac{5}{36}$ & $\frac{5}{63}$ & \\
\hline $\begin{array}{llll}0 & 1 & -1 & \frac{1}{2}\end{array}$ & $\frac{4}{45}$ & $\frac{1}{9}$ & $\frac{1}{4}$ & $\frac{27}{140}$ & $\frac{5}{18}$ & $\frac{5}{63}$ & $\frac{1}{72}$ & $\frac{5}{32}$ & $-\frac{27}{224}$ & $-\frac{5}{36}$ & $\frac{25}{144}$ & $\frac{\frac{50}{63}}{63}$ & \\
\hline $\begin{array}{llll}-1 & \frac{1}{2} & 0 & 1\end{array}$ & $\frac{2}{135}$ & $-\frac{49}{216}$ & $\frac{3}{32}$ & $-\frac{361}{1120}$ & $\frac{125}{432}$ & $-\frac{10}{189}$ & $-\frac{1}{27}$ & 0 & $\frac{1}{7}$ & $-\frac{5}{54}$ & $-\frac{25}{54}$ & $\frac{50}{189}$ & \\
\hline $\begin{array}{llll}-1 & \frac{3}{2} & 0 & 1\end{array}$ & $\frac{8}{27}$ & $\frac{10}{27}$ & 0 & $-\frac{2}{7}$ & $-\frac{1}{27}$ & $-\frac{2}{189}$ & $-\frac{25}{216}$ & $\frac{15}{32}$ & $-\frac{25}{224}$ & $\frac{5}{27}$ & $-\frac{49}{432}$ & $-\frac{1}{189}$ & $-\frac{1}{3}$ \\
\hline $\begin{array}{llll}0 & 2 & -1 & \frac{1}{2}\end{array}$ & & & & & & & $\frac{25}{72}$ & $\frac{5}{32}$ & $\frac{75}{224}$ & $\frac{5}{36}$ & $\frac{1}{144}$ & $\frac{1}{63}$ & $\frac{2}{3}$ \\
\hline $\begin{array}{llll}1 & \frac{3}{2} & 0 & 0\end{array}$ & & & & & & & $-\frac{5}{24}$ & & $\frac{5}{224}$ & $\frac{\bar{z}}{3}$ & $\frac{5}{48}$ & $\frac{5}{21}$ & \\
\hline
\end{tabular}

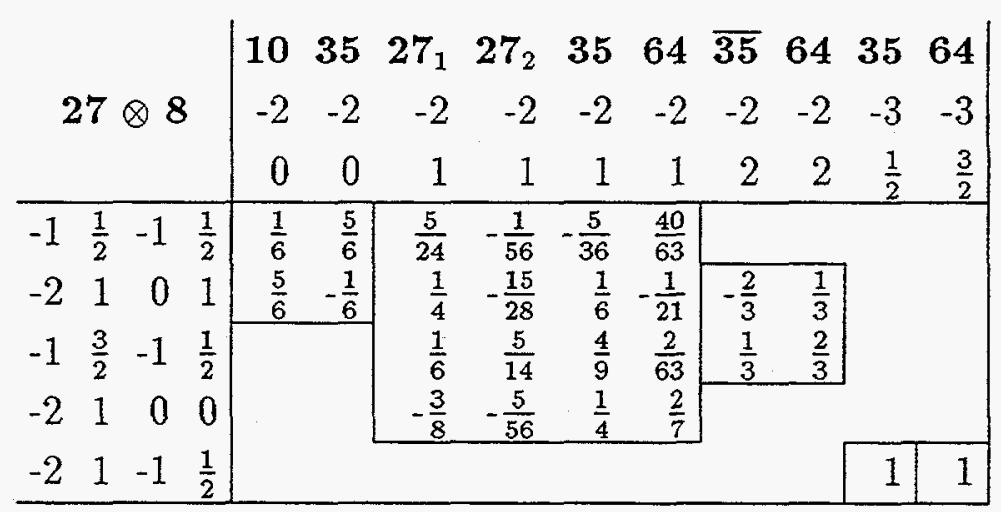


Table 3.14: Isoscalar factors for $\mathbf{2 7} \otimes \mathbf{2 7}$ to the singlet only.

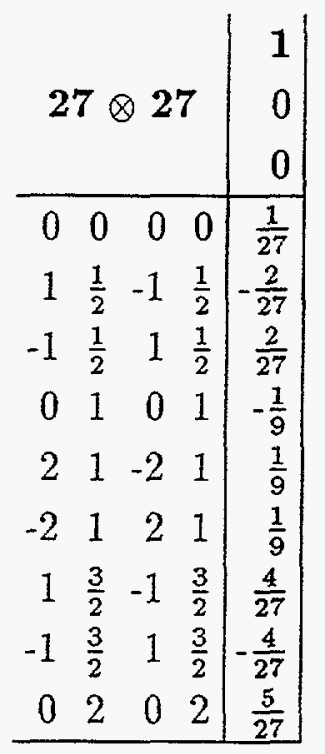




\section{$4 \quad D$-Meson Decays in Broken Flavor $S U(3)$}

Many data are available on the hadronic two-body decays of charmed mesons. Theoretical models that attempt to systematize the decay patterns have been available for many years. These models usually make dynamical assumptions in order to reduce the number of amplitudes that contribute to a particular decay. For example, the large $N_{c}$ approximation [46] [47], or the heavy-quark effective theory [48]. It is not clear a priori how well such approximations should work and hence how seriously to take a conflict between a prediction and a measured value. Another approach is to assume that the matrix elements factorize [49]. This model is quite successful in describing observed modes, but again, it is difficult to know whether a discrepancy is due to an incorrect measurement of the failure of the assumption. A more general approach based on a diagrammatic classification [50], with different assumptions, also exists. In many cases attempts are made to obtain predictions of unmeasured modes from these models.

$S U(3)$ is badly broken in these decays, so models based on exact symmetry [51] are not useful. An analysis of the $S U(3)$ breaking was begun in [52]. However, an attempt at a complete parameterization has been conspicuously missing, due to the large number of reduced matrix elements involved. We set out to remedy this omission. This chapter gives a full parameterization of the decays of the $D$ mesons into final states of two pseudoscalars (PP), two vectors (VV) and a pseudoscalar plus a vector (PV), including $S U(3)$-breaking. The elements of this parameterizationthe particle representations, the weak hamiltonian, the breaking operator, and the 
reduced matrix elements-are discussed in the following sections. We make only very few assumptions to limit the number of parameters. We fit the parameters to the available data of two-body decays and predict many unmeasured modes. Because a few of the parameters are not corstrained, we indicate which branching fractions are needed to predict the rest of certain classes of modes. We comment on the case of $D_{s} \rightarrow \eta^{\prime} \rho^{+}$, where the model is barely consistent with data. Much of this analysis can be found in [53].

\subsection{Particle States in Flavor $S U(3)$}

In a model based on flavor $S U(3)$, the particles are denoted by their $S U(3)$ representation. The fundamental representation is the triplet (3) of quarks $u, d$, and $s$. The three $D$ mesons $\left\{D^{0}, D^{+}, D_{s}^{+}\right\}$form an antitriplet $(\overline{3})$ representation. The pseudoscalars $\left\{\pi^{+}, \pi^{0}, \pi^{-}, K^{+}, K^{0}, K^{-}, \bar{K}^{0}, \eta_{8}\right\}$ form an octet (8) representation, as do the vectors $\left\{\rho^{+}, \rho^{0}, \rho^{-}, K^{*+}, K^{* 0}, \overline{K^{*-}}, \overline{K^{* 0}}, \omega_{8}\right\}$. The $\eta_{1}$ and $\omega_{1}$ are each singlets.

$\begin{aligned} D & \sim \overline{3} \\ P & \sim 8, \\ V & \sim 8, \\ \eta_{1} & \sim 1, \\ \omega_{1} & \sim 1 .\end{aligned}$

The physical $\eta, \eta^{\prime}, \phi$, and $\omega$ are linear combinations of singlets and octets:

$$
\begin{aligned}
\eta & =\cos \theta_{P} \eta_{8}-\sin \theta_{P} \eta_{1}, \\
\eta^{\prime} & =\sin \theta_{P} \eta_{8}+\cos \theta_{P} \eta_{1}, \\
\omega & =\cos \theta_{V} \omega_{8}-\sin \theta_{V} \omega_{1}, \\
\phi & =\sin \theta_{V} \omega_{8}+\cos \theta_{V} \omega_{1},
\end{aligned}
$$

with mixing angles $\theta_{p}=-17.3^{\circ}[54]$ and $\theta_{V}=39^{\circ}$ [10]. Throughout this chapter, $K^{*}$ will always denote $K^{*}(892)$ and $\eta^{\prime}$ will denote $\eta^{\prime}(958)$. 


\subsection{The Weak Hamiltonian}

The decays of the $D$ mesons are mediated by the weak hamiltonian. Ignoring QCD corrections, the hamiltonian in terms of the quark fields is

$$
\begin{aligned}
& H_{\text {weak }}=\quad \frac{G_{F}}{\sqrt{2}} \cos ^{2} \theta_{C} \bar{u} \gamma^{\mu}\left(1-\gamma_{5}\right) d \bar{s} \gamma_{\mu}\left(1-\gamma_{5}\right) c \\
& +\frac{G_{F}}{\sqrt{2}} \cos \theta_{C} \sin \theta_{C} \bar{u} \gamma^{\mu}\left(1-\gamma_{5}\right) s \bar{s} \gamma_{\mu}\left(1-\gamma_{5}\right) c \\
& -\frac{G_{F}}{\sqrt{2}} \cos \theta_{C} \sin \theta_{C} \bar{u} \gamma^{\mu}\left(1-\gamma_{5}\right) d \bar{d} \gamma_{\mu}\left(1-\gamma_{5}\right) c \\
& -\frac{G_{F}}{\sqrt{2}} \sin ^{2} \theta_{C} \bar{u} \gamma^{\mu}\left(1-\gamma_{5}\right) s \bar{d} \gamma_{\mu}\left(1-\gamma_{5}\right) c .
\end{aligned}
$$

Note that the operators $\bar{q}$ create quarks and so transform as a triplet, while $q$ transforms as the antitriplet.

\subsection{1 $H_{\text {weak }}$ in Terms of $S U(3)$ Representations}

Using the Clebsch-Gordan coefficients for the expansion of the product $\mathbf{3} \times \overline{\mathbf{3}} \times \mathbf{3}$, we can classify the operators according to irreducible representations of $S U(3)$ as follows:

$$
\begin{aligned}
& (\bar{u} d)(\bar{s} c)=\frac{1}{\sqrt{2}} \overline{\mathbf{6}}\left(-\frac{2}{3}, 1,1\right)+\frac{1}{\sqrt{2}} \mathbf{1 5}\left(-\frac{2}{3}, 1,1\right) \\
& (\bar{u} s)(\bar{d} c)=-\frac{1}{\sqrt{2}} \overline{\mathbf{6}}\left(\frac{4}{3}, 0,0\right)+\frac{1}{\sqrt{2}} \mathbf{1 5}\left(\frac{4}{3}, 1,0\right) \\
& (\bar{u} d)(\bar{d} c)=\sqrt{\frac{3}{8}} \mathbf{3}\left(\frac{1}{3}, \frac{1}{2}, \frac{1}{2}\right)-\frac{1}{2} \overline{\mathbf{6}}\left(\frac{1}{3}, \frac{1}{2}, \frac{1}{2}\right)+\frac{1}{\sqrt{3}} \mathbf{1 5}\left(\frac{1}{3}, \frac{3}{2}, \frac{1}{2}\right)-\frac{1}{\sqrt{24}} \mathbf{1 5}\left(\frac{1}{3}, \frac{1}{2}, \frac{1}{2}\right) \\
& (\bar{u} s)(\bar{s} c)=\sqrt{\frac{3}{8}} \mathbf{3}\left(\frac{1}{3}, \frac{1}{2}, \frac{1}{2}\right)+\frac{1}{2} \overline{\mathbf{6}}\left(\frac{1}{3}, \frac{1}{2}, \frac{1}{2}\right)+\sqrt{\frac{3}{8}} \mathbf{1 5}\left(\frac{1}{3}, \frac{1}{2}, \frac{1}{2}\right)
\end{aligned}
$$

where $\left(\bar{q} q^{\prime}\right)$ denotes $\bar{q} \gamma^{\mu}\left(1-\gamma_{5}\right) q^{\prime}$. The numbers in parentheses are hypercharge, total isospin, and third component of isospin of the particular members of the $S U(3)$ representations. The weak hamiltonian can now be written in terms of the representations $\mathbf{3}, \mathbf{3}^{\prime}, \overline{\mathbf{6}}$, and $\mathbf{1 5}$ as

$$
\begin{aligned}
H_{\mathrm{weak}}= & G_{F} \sin ^{2} \theta_{C}\left[\frac{1}{2} \overline{6}\left(\frac{4}{3}, 0,0\right)-\frac{1}{2} 15\left(\frac{4}{3}, 1,0\right)\right] \\
+ & G_{F} \cos ^{2} \theta_{C}\left[\frac{1}{2} \overline{6}\left(-\frac{2}{3}, 1,1\right)+\frac{1}{2} 15\left(-\frac{2}{3}, 1,1\right)\right] \\
+ & G_{F} \cos \theta_{C} \sin \theta_{C} \\
& {\left[\frac{1}{\sqrt{2}} \overline{6}\left(\frac{1}{3}, \frac{1}{2}, \frac{1}{2}\right)-\frac{1}{\sqrt{6}} 15\left(\frac{1}{3}, \frac{3}{2}, \frac{1}{2}\right)+\frac{1}{\sqrt{3}} 15\left(\frac{1}{3}, \frac{1}{2}, \frac{1}{2}\right)\right] . }
\end{aligned}
$$


The combination of $\mathbf{1 5}\left(\frac{1}{3}, \frac{3}{2}, \frac{1}{2}\right)$ and $\mathbf{1 5}\left(\frac{1}{3}, \frac{1}{2}, \frac{1}{2}\right)$ is the center member of a $U$-spin vector, as discussed in [51]. Note that the 3 does not appear in $H_{\text {weak }}\left(M_{W}\right)$ [55]. Because the QCD corrections are multiplicative and do not mix the $S U(3)$ representations, the 3 will also not appear in $H_{\text {weak }}\left(m_{c}\right)$.

\subsubsection{QCD Evolution}

Since the decays of the $D$ mesons occur at the scale of the $c$-quark mass, we must allow the QCD evolution of the various operators from the $W$-mass scale, where Equation 4.3 is valid, to the $c$-mass scale. The operators represented by the $\mathbf{1 5}$ are symmetric under quark interchange, and those represented by the $\overline{6}$ are antisymmetric. The QCD renormalization of operators with these symmetry properties has been calculated [56].

We find that

$$
\begin{array}{r}
15 \rightarrow 15 \times\left[\frac{\alpha_{s}\left(M_{W}\right)}{\alpha_{s}\left(m_{b}\right)}\right]^{a_{5}^{+}} \times\left[\frac{\alpha_{s}\left(m_{b}\right)}{\alpha_{s}\left(m_{c}\right)}\right]^{a_{4}^{+}} \\
\overline{6} \rightarrow \overline{6} \times\left[\frac{\alpha_{s}\left(M_{W}\right)}{\alpha_{s}\left(m_{b}\right)}\right]^{a_{5}^{-}} \times\left[\frac{\alpha_{s}\left(m_{b}\right)}{\alpha_{s}\left(m_{c}\right)}\right]^{a_{4}^{-}},
\end{array}
$$

where

$$
\begin{aligned}
& a_{N_{f}}^{+}=\frac{6}{33-2 N_{f}}, \\
& a_{N_{f}}=\frac{-12}{33-2 N_{f}},
\end{aligned}
$$

in the regime where there are $N_{f}$ flavor degrees of freedom. Taking into account the change in the number of active flavors as the $b$-quark threshold is crossed, and using $\alpha_{s}\left(M_{Z}\right)=0.116[10]$, we obtain

$$
\begin{aligned}
& 15 \rightarrow 0.818 \mathbf{1 5}, \\
& \overline{\mathbf{6}} \rightarrow 1.49 \overline{\mathbf{6}} \text {. }
\end{aligned}
$$

With Equation 4.5 as the boundary condition, we have

$$
\begin{aligned}
H_{\mathrm{weak}}\left(m_{c}\right)= & \frac{G_{F}}{2} \sin ^{2} \theta_{C}\left[-0.818 \mathbf{1 5}\left(\frac{4}{3}, 1,0\right)+1.49 \overline{\mathbf{6}}\left(\frac{4}{3}, 0,0\right)\right] \\
+ & \frac{G_{F}}{2} \cos ^{2} \theta_{C}\left[0.818 \mathbf{1 5}\left(-\frac{2}{3}, 1,1\right)+1.49 \overline{\mathbf{6}}\left(-\frac{2}{3}, 1,1\right)\right] \\
+ & \frac{G_{F}}{2} \cos \theta_{C} \sin \theta_{C}\left[0.818 \times \frac{2}{\sqrt{3}} \mathbf{1 5}\left(\frac{1}{3}, \frac{1}{2}, \frac{1}{2}\right)\right. \\
& \left.-0.818 \times \sqrt{\frac{2}{3}} \mathbf{1 5}\left(\frac{1}{3}, \frac{3}{2}, \frac{1}{2}\right)+1.49 \times \sqrt{2} \overline{6}\left(\frac{1}{3}, \frac{1}{2}, \frac{1}{2}\right)\right] .
\end{aligned}
$$


Note that the QCD corrections do not introduce any new phases into the process. Unfortunately, until the values of the reduced matrix elements (discussed below) are known, the coefficients in Equation 4.9 are of little use.

\subsection{Parameterization}

\subsection{1 $S U(3)$ Breaking}

For a complete parameterization of any process in flavor $S U(3)$, we must include explicit breaking. Since we know that the source of flavor $S U(3)$ breaking among the pions and kaons is the difference between the quark masses, we do this with an operator $M$ which transforms as an 8 .

We can express $M$ as

$$
M=\alpha \lambda_{3}+\beta \lambda_{8}
$$

where $\lambda_{i}$ are the usual Gell-Mann matrices. The term in $\alpha$ represents breaking of the isospin $S U(2)$ subgroup. This breaking, proportional to the difference between up and down quark masses, is expected to be very small and we neglect it in the following. When allowed to vary, the data prefers a small value for $\alpha$. The constant $\beta$ can be absorbed into the reduced matrix elements. Hence $M$ can be reduced to

$$
M=\lambda_{8}
$$

\subsubsection{Reduced Matrix Elements}

Now consider the most general parameterization of the decays in the context of the flavor $S U(3)$ symmetry. For each possible contraction of the representations into an $S U(3)$ singlet there must be one parameter, i.e., one reduced matrix element. The representations involved are those in Section 4.1: $D(\overline{\mathbf{3}}), H(\overline{\mathbf{6}}+\mathbf{1 5})$, and two of $\mathrm{P}$ and $\mathrm{V}$ (each 1 or 8 ). In addition, we must include all possible ways of involving the symmetry-breaking parameter $M$. We assume that the breaking is linear in $M$. 
Each reduced matrix element is, in principle, complex. We have chosen to contract $D$ with $H$, then contract the products (PP, PV, VV) (and then possibly with $M$ ), and finally contract the two parts into the singlet. Our labels for the reduced matrix elements reflect this. For example, the matrix element denoted $\left(D H_{15}\right)_{8}\left((\mathrm{PP})_{1} M\right)_{8}$ is obtained by contracting $D$ and the $\mathbf{1 5}$ component of $H$ into an octet, contracting PP into a singlet which combines with $M$ to become another octet, and contracting the two resulting octets into the singlet.

Unfortunately, the above parameterization involves far more parameters than there exist data. Therefore we make two important assumptions. First, we assume that we can separate the spin and flavor dynamics of the processes, i.e., that the relative strengths of the reduced matrix elements are the same in the PP, PV, and VV cases. This implies that only forty-eight reduced $S U(3)$ matrix elements are needed. They are labeled with $S$ and $O$ for the singlet and octet representations, rather than with $\mathrm{PP}, \mathrm{PV}$, or VV. In order to distinguish the spin states we introduce two parameters, called "PV" and "VV." Second, we assume that the phase of each reduced matrix element is given solely by the representation of the product particles (before $M$ is included). Bose symmetry for PP and VV and an appropriate phase rotation of the particle fields reduces the list of phases to $\left(\eta_{1} \eta_{1}\right)_{1},\left(\eta_{1} \omega_{1}\right)_{1}$, $\left(\omega_{1} \omega_{1}\right)_{1},\left(\mathrm{P} \eta_{1}\right)_{8},\left(\mathrm{P} \omega_{1}\right)_{8},\left(\mathrm{~V} \eta_{1}\right)_{8},\left(\mathrm{~V} \omega_{1}\right)_{8},(\mathrm{PP})_{1},(\mathrm{PP})_{27},(\mathrm{PV})_{1},(\mathrm{PV})_{8^{\prime}},(\mathrm{PV})_{10}$ $(\mathrm{PV})_{\overline{10}},(\mathrm{PV})_{27},(\mathrm{VV})_{1}$, and $(\mathrm{VV})_{27}$. One should note that we cannot determine the relative phases between PP, PV, and VV. The complete list of parameters appears in Tables 4.2 and 4.3.

The amplitude for each decay mode can be expressed as a sum over the reduced matrix elements with the appropriate Clebsch-Gordan coefficients:

$$
A\left(D_{j} \rightarrow X_{i}\right)=\sum_{k} C_{i j k} R_{k} S_{i}
$$

Here $R_{k}$ are the reduced $S U(3)$ matrix elements and $S_{i}$ are the parameters that we call PP $\equiv 1, \mathrm{PV}$, and VV. The $S U(3)$ Clebsch-Gordan factors $C_{i}$ were calculated by computer. Many of the routines used are described in Chapter 3. 
Table 4.1: $\quad$ List of reduced matrix elements.

\begin{tabular}{|l|l|}
\hline$\left(D H_{\overline{6}}\right)_{8}(S S M)_{8}$ & $\left(D H_{15}\right)_{8}(S S M)_{8}$ \\
\hline$\left(D H_{\overline{6}}\right)_{8}(S O)_{8}$ & $\left(D H_{15}\right)_{8}(S O)_{8}$ \\
$\left(D H_{\overline{6}}\right)_{8}(S O M)_{8}$ & $\left(D H_{\overline{6}}\right)_{8}(S O M)_{8^{\prime}}$ \\
$\left(D H_{\overline{6}}\right)_{\overline{10}}(S O M)_{10}$ & $\left(D H_{15}\right)_{8}(S O M)_{8}$ \\
$\left(D H_{15}\right)_{8}(S O M)_{8^{\prime}}$ & $\left(D H_{15}\right)_{10}(S O M)_{\overline{10}}$ \\
$\left(D H_{15}\right)_{27}(S O M)_{27}$ & \\
\hline$\left(D H_{\overline{6}}\right)_{8}(O O)_{8}$ & $\left(D H_{\overline{6}}\right)_{8}(O O)_{8^{\prime}}$ \\
$\left(D H_{\overline{6}}\right)_{\overline{10}}(O O)_{10}$ & $\left(D H_{15}\right)_{8}(O O)_{8}$ \\
$\left(D H_{15}\right)_{8}(O O)_{8^{\prime}}$ & $\left(D H_{15}\right)_{10}(O O)_{\overline{10}}$ \\
$\left(D H_{15}\right)_{27}(O O)_{27}$ & $\left(D H_{\overline{6}}\right)_{8}\left((O O)_{1} M\right)_{8}$ \\
$\left(D H_{\overline{6}}\right)_{8}\left((O O)_{8} M\right)_{8}$ & $\left(D H_{\overline{6}}\right)_{8}\left((O O)_{8} M\right)_{8^{\prime}}$ \\
$\left(D H_{\overline{6}}\right)_{\overline{10}}\left((O O)_{8} M\right)_{10}$ & $\left(D H_{\overline{6}}\right)_{8}\left((O O)_{8^{\prime}} M\right)_{8}$ \\
$\left(D H_{\overline{6}}\right)_{8}\left((O O)_{8^{\prime}} M\right)_{8^{\prime}}$ & $\left(D H_{\overline{6}}\right)_{\overline{10}}\left((O O)_{8^{\prime}} M\right)_{10}$ \\
$\left(D H_{\overline{6}}\right)_{8}\left((O O)_{10} M\right)_{8}$ & $\left(D H_{\overline{6}}\right)_{\overline{10}}\left((O O)_{10} M\right)_{10}$ \\
$\left(D H_{\overline{6}}\right)_{8}\left((O O)_{\overline{10}} M\right)_{8}$ & $\left(D H_{\overline{6}}\right)_{8}\left((O O)_{27} M\right)_{8}$ \\
$\left(D H_{\overline{6}}\right)_{\overline{10}}\left((O O)_{27} M\right)_{10}$ & $\left(D H_{15}\right)_{8}\left((O O)_{1} M\right)_{8}$ \\
$\left(D H_{15}\right)_{8}\left((O O)_{8} M\right)_{8}$ & $\left(D H_{15}\right)_{8}\left((O O)_{8} M\right)_{8^{\prime}}$ \\
$\left(D H_{15}\right)_{10}\left((O O)_{8} M\right)_{\overline{10}}$ & $\left(D H_{15}\right)_{27}\left((O O)_{8} M\right)_{27}$ \\
$\left(D H_{15}\right)_{8}\left((O O)_{8^{\prime}} M\right)_{8}$ & $\left(D H_{15}\right)_{8}\left((O O)_{8^{\prime}} M\right)_{8^{\prime}}$ \\
$\left(D H_{15}\right)_{10}\left((O O)_{8^{\prime}} M\right)_{\overline{10}}$ & $\left(D H_{15}\right)_{27}\left((O O)_{8^{\prime}} M\right)_{27}$ \\
$\left(D H_{15}\right)_{8}\left((O O)_{10} M\right)_{8}$ & $\left(D H_{15}\right)_{27}\left((O O)_{10} M\right)_{27}$ \\
$\left(D H_{15}\right)_{8}\left((O O)_{\overline{10}} M\right)_{8}$ & $\left(D H_{15}\right)_{10}\left((O O)_{\overline{10}} M\right)_{\overline{10}}$ \\
$\left(D H_{15}\right)_{27}\left((O O)_{\overline{10}} M\right)_{27}$ & $\left(D H_{15}\right)_{8}\left((O O)_{27} M\right)_{8}$ \\
$\left(D H_{15}\right)_{10}\left((O O)_{27} M\right)_{\overline{10}}$ & $\left(D H_{15}\right)_{27}\left((O O)_{27} M\right)_{27}$ \\
$\left(D H_{15}\right)_{27}\left((O O)_{27} M\right)_{27^{\prime}}$ & \\
\hline
\end{tabular}




\subsubsection{Linear Combinations of Reduced Matrix Elements}

There are 45 measured values for the two-body decay modes and an additional 13 modes where upper limits exist. ${ }^{4}$ It would appear that there are still more parameters than data, and therefore the model lacks predictability. However, there are only forty linearly independent combinations of the $S U(3)$ reduced matrix elements that contribute to the possible decay modes of the $D$ mesons. With the assumption of the last section concerning the phases of the reduced matrix elements, the linear combinations fall into these classes:

$$
\begin{array}{ll}
\text { involving }(S S)_{1}: & L^{(1)} \\
\text { involving }(S O)_{8}: & L^{(2)}, \ldots, L^{(8)} \\
\text { involving }(O O)_{1}: & L^{(9)} \\
\text { involving }(O O)_{8}: & L^{(10)}, \ldots, L^{(16)} \\
\text { involving }(O O)_{8^{\prime}}: & L^{(17)}, \ldots, L^{(23)} \\
\text { involving }(O O)_{10}: & L^{(24)}, \ldots, L^{(28)} \\
\text { involving }(O O)_{\overline{10}}: & L^{(29)}, \ldots, L^{(33)} \\
\text { involving }(O O)_{27}: & L^{(34)}, \ldots, L^{(40)}
\end{array}
$$

We write them each as a sum over the reduced matrix elements, viz.,

$$
L^{(n)}=\sum_{i} C_{i n}^{\prime} R_{i}
$$

and normalize them for convenience by setting

$$
\sum_{i} C_{i n}^{\prime 2}=1
$$

Now Equation 4.12 is replaced by

$$
A\left(D_{j} \rightarrow X_{i}\right)=\sum_{n} C_{i j n}^{\prime \prime} L^{(n)} S_{i}
$$

${ }^{4}$ The data are from the Particle Data Group [10], together with [57] for the mode $D^{+} \rightarrow K^{* 0} \pi^{+}$. 
The $L^{(n)}$ replace the reduced matrix elements in our parameterization of the amplitudes. The forty linearly independent combinations contain matrix elements including those that involve the breaking operator $M$. It is not possible to divide the linear combinations into a set that contains only matrix elements without $M$ and a set containing only matrix elements with $M$. Of the forty combinations, three are not constrained by the available data. We call them $L^{(1)}, L^{(2)}$, and $L^{(3)}$. They are discussed below.

The replacement of the set of reduced matrix elements by the set of linear combinations that contribute to the possible decay modes reduces the number of parameters by eight. The total number is now fifty three. These parameters are fit to the data; the individual reduced matrix elements are no longer considered. The values of the linear combinations for the best fit are in Table 4.2. The signs have been absorbed into the $C_{i j n}^{\prime \prime}$. The remaining parameters are in Table 4.3. All phases are given in radians. The uncertainties on the fit values of the parameter correspond to one unit of $\chi^{2}$.

The unconstrained combination $L^{(1)}$ contributes to the modes $D^{0} \rightarrow \eta \eta, \eta \eta^{\prime}$, $\eta^{\prime} \eta^{\prime}, \eta \phi, \eta \omega, \eta^{\prime} \phi, \eta^{\prime} \omega, \phi \phi, \phi \omega$, and $\omega \omega$. Because these modes are unobserved, the phases of $\left(\eta_{1} \omega_{1}\right)_{1}$, and $\left(\omega_{1} \omega_{1}\right)_{1}$ are also unconstrained. The remaining unconstrained linear combinations are $L^{(2)}$ and $L^{(3)}$. They contribute to the above modes, and also to modes of the types $D^{0} \rightarrow \eta K^{0}$ and $D_{s} \rightarrow \eta K^{+}$. By "type" we mean a class of modes that contain mesons of the same flavors and charges. Thus the type $D_{s} \rightarrow \eta K^{+}$contains the modes $D_{s} \rightarrow \eta K^{+}, \eta^{\prime} K^{+}, \eta K^{*+}, \eta^{\prime} K^{*+}, \phi K^{+}, \omega K^{+}, \phi K^{*+}$, $\omega K^{*+}$, and no others. With the exception of the limit on the branching ratio for $D_{s} \rightarrow \phi K^{+}$, there are no data for these modes. We still have some freedom in the definition of $L^{(2)}$ and $L^{(3)}$ that allows modes of the type $D^{0} \rightarrow \eta K^{0}$ to depend on only one of them (choose $L^{(2)}$ ). This will allow us to estimate one of their branching fractions and thereby make some predictions of the other modes of this type. 


\subsection{Data and Fitting Thereof}

The data used to determine the parameters are listed in Tables 4.4-4.7. These are the modes for which there exist either experimental values or experimental limits. In the VV modes, S and D waves are possible. Data exist from $\mathrm{E} 691$ [58] for the modes $D^{0} \rightarrow \bar{K}^{* 0} \rho^{0}$ and $D^{+} \rightarrow \bar{K}^{* 0} \rho^{+}$. These are consistent with the S- and $\mathrm{D}$-waves both having significant amplitudes and are inconsistent with either being zero. The ratios of $\mathrm{S}$ - and $\mathrm{D}$-wave amplitudes from these two modes are taken as additional data, and the overall ratio of $\mathrm{S}$ - to $\mathrm{D}$-wave amplitudes for the VV modes is allowed to vary in the fit. Its value is determined by the two modes mentioned above, and depends very little on the other data.

For each mode we remove the phase space and Cabibbo factors and reduce the brariching ratio to a decay amplitude in arbitrary units. Because the vector particles have substantial widths, the phase space for modes involving a vector is integrated over the relativistic Breit-Wigner for that resonance. The effect of this is important for those modes where the sum of the particle masses is within a few widths of the $D$ mass. The modes $D^{0} \rightarrow \phi K^{* 0}, \phi \bar{K}^{* 0}$, and $D^{+} \rightarrow \phi K^{*+}$ would be forbidden if the widths were set to zero. Each amplitude is now expressed as a sum of Clebsch-Gordan coefficients times the parameters that represent the reduced matrix elements, and finally as a.sum over the linearly independent combinations of reduced matrix elements.

The parameters were fit to the data amplitudes with MINUIT, release 93.11, suitably modified to accomodate a larger number of parameters. The total $\chi^{2}$ was found to be 30.9 for seven degrees of freedom, indicating that the overall fit was poor. However, more than half of the $\chi^{2}$ arose from only one mode. The mode in question is $D_{s} \rightarrow \eta^{\prime} \rho^{+}$. The experimental value for the branching ratio $D_{s} \rightarrow \eta^{\prime} \rho$ cannot be accommodated in our scheme. It is measured [59] to be larger than that for $D_{s} \rightarrow \eta \rho$, an a priori surprising result. We note that the angular distribution of the decay pions is barely consistent with that expected. The expected distribution for 
a spin-1 particle is a $\left|Y_{1}^{1}\right|^{2}=\cos ^{2} \theta$ above a flat background, where $\theta$ is the "helicity angle." It is the angle between the momentum of the final $\pi^{+}$to the momentum of the reconstructed $\rho^{+}$. A spin-2 resonance will add a $\left|Y_{2}^{2}\right|^{2}$ component to the distribution. When we refit the data reported in [59], we find that better agreement is obtained if $30 \%$ of the events above the background is due to a spin- 2 object. See Figure 4.1. A confirmation of this experimental value would be very significant, as all other models [49] also predict a ratio of $B R\left(D_{s} \rightarrow \eta^{\prime} \rho\right) / B R\left(D_{s} \rightarrow \eta \rho\right)$ of less than one.

We decided to reject the experimental value for the branching fraction of $D_{s} \rightarrow$ $\eta^{\prime} \rho^{+}$. The result is a better fit, from which the branching ratios are reported in the tables. The total $\chi^{2}$ is now 11.6 for six degrees of freedom. 
Figure 4.1: Number of events versus helicity angle in the analysis of the decay $D_{s} \rightarrow \eta^{\prime} \rho^{+}[59]$. The dashed curve is a fit to spin-1 plus flat background (the background is removed). The solid curve includes a spin-2 component. See Section 4.4 of the text.

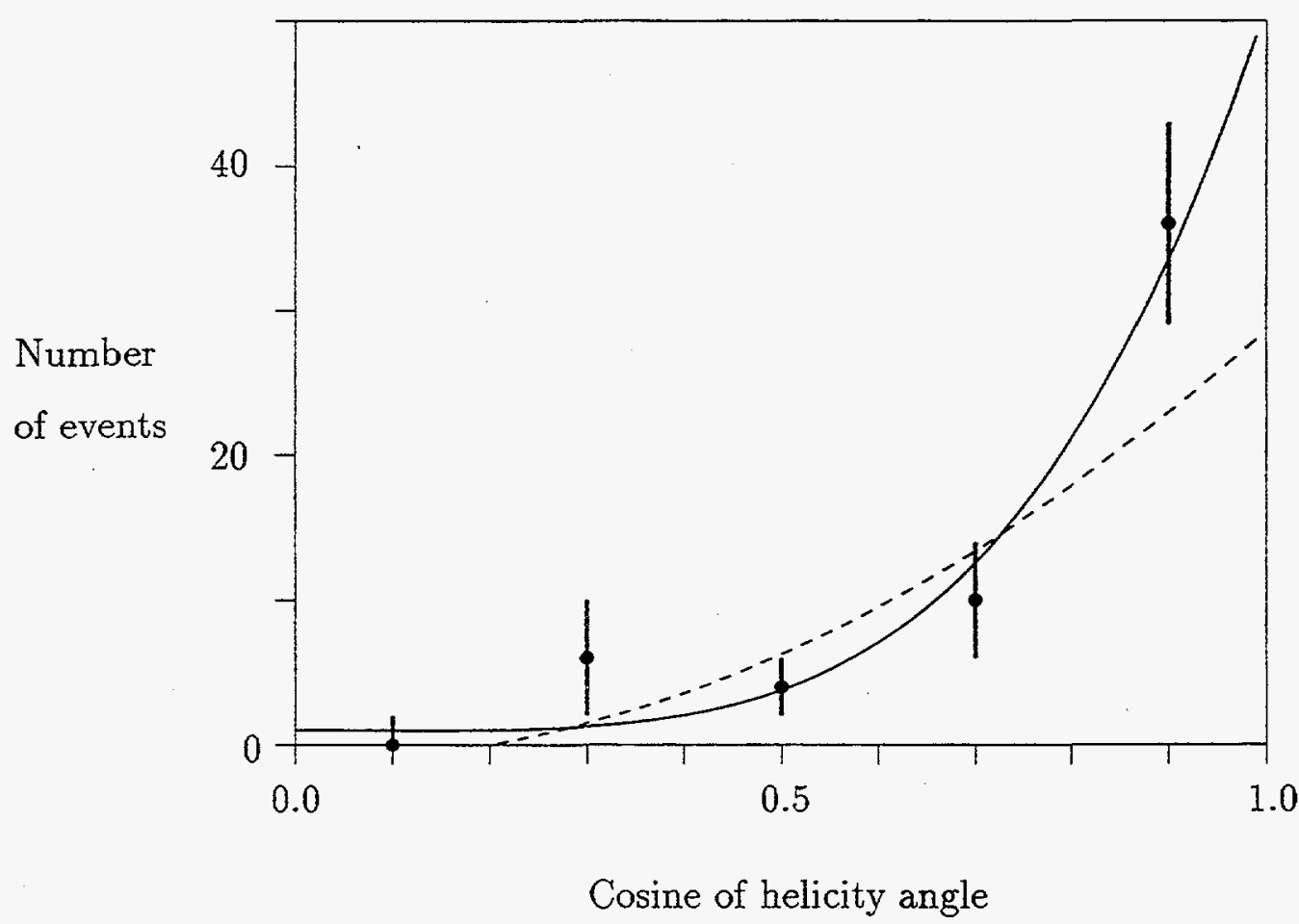


Table 4.2: $\quad$ Linear combinations and their fit values.

\begin{tabular}{|c|c|c|c|c|c|}
\hline $\begin{array}{l}\text { Linear } \\
\text { combination }\end{array}$ & $\begin{array}{l}\text { Amplitudes } \\
\text { involved }\end{array}$ & Fit value & & & \\
\hline$L^{(1)}$ & $(S S)_{1}$ & - & & & \\
\hline$L^{(2)}$ & & - & Linear & Amplitudes & Fit value \\
\hline$L^{(3)}$ & & - & combination & involved & \\
\hline$L^{(4)}$ & & $2.97 \pm 0.66$ & $L^{(24)}$ & & $4.7 \pm 5.9$ \\
\hline$L^{(5)}$ & $(S O)_{8}$ & $6 \pm 26$ & $L^{(25)}$ & & $59 \pm 44$ \\
\hline$L^{(6)}$ & & $8 \pm 17$ & $L^{(26)}$ & $(O O)_{10}$ & $107 \pm 63$ \\
\hline$L^{(7)}$ & & $16 \pm 18$ & $L^{(27)}$ & & $39 \pm 21$ \\
\hline$L^{(8)}$ & & $6 \pm 13$ & $L^{(28)}$ & & $43 \pm 37$ \\
\hline$L^{(9)}$ & $(O O)_{1}$ & $2.4 \pm 2.7$ & $L^{(29)}$ & & $21.1 \pm 7.1$ \\
\hline$L^{(10)}$ & & $5.20 \pm 0.12$ & $L^{(30)}$ & & $71 \pm 67$ \\
\hline$L^{(11)}$ & & $1.7 \pm 7.3$ & $L^{(31)}$ & $(00)_{\overline{10}}$ & $97 \pm 51$ \\
\hline$L^{(12)}$ & & $12.4 \pm 2.3$ & $L^{(32)}$ & & $135 \pm 74$ \\
\hline$L^{(13)}$ & $(00)_{8}$ & $34 \pm 12$ & $L^{(33)}$ & & $115 \pm 61$ \\
\hline$L^{(14)}$ & & $9.5 \pm 5.5$ & $L^{(34)}$ & & $0.4 \pm 2.5$ \\
\hline$L^{(15)}$ & & $45 \pm 21$ & $L^{(35)}$ & & $26.2 \pm 7.3$ \\
\hline$L^{(16)}$ & & $52 \pm 21$ & $L^{(36)}$ & & $2.5 \pm 2.9$ \\
\hline$L^{(\mathbf{1 7})}$ & & $10.8 \pm 4.1$ & $L^{(37)}$ & $(O O)_{27}$ & $21.8 \pm 4.6$ \\
\hline$L^{(18)}$ & & $55 \pm 37$ & $L^{(38)}$ & & $9.2 \pm 6.2$ \\
\hline$L^{(19)}$ & & $44 \pm 38$ & $L^{(39)}$ & & $8.0 \pm 8.6$ \\
\hline$L^{(20)}$ & $(O O)_{8^{\prime}}$ & $45 \pm 44$ & $L^{(40)}$ & & $19 \pm 11$ \\
\hline$L^{(21)}$ & & $34 \pm 25$ & & & \\
\hline$L^{(22)}$ & & $42 \pm 46$ & & & \\
\hline$L^{(23)}$ & & $11 \pm 47$ & & & \\
\hline
\end{tabular}


$\because \quad$ Table 4.3: The remaining parameters and their fit values.

\begin{tabular}{|c|c|}
\hline Parameter & Fit value \\
\hline$P V$ & $0.91 \pm 0.32$ \\
\hline VV & $1.40 \pm 0.16$ \\
\hline D-/S-wave & $2.79 \pm 0.41$ \\
\hline phase of $\eta_{1} \eta_{1}$ & - \\
\hline phase of $\mathrm{P} \eta_{1}$ & $1.64 \pm 0.45$ \\
\hline phase of $\mathrm{PP}_{1}$ & $0.7 \pm 1.2$ \\
\hline phase of $\mathrm{PP}_{27}$ & $1.49 \pm 0.16$ \\
\hline phase of $\omega_{1} \omega_{1}$ & - \\
\hline phase of $V \omega_{1}$ & $1.28 \pm 0.52$ \\
\hline phase of $\mathrm{VV}_{1}$ & $-3.0 \pm 3.2$ \\
\hline phase of $V V_{27}$ & $0.53 \pm 0.77$ \\
\hline phase of $\eta_{1} \omega_{1}$ & - \\
\hline phase of $P \omega_{1}$ & $0.01 \pm 0.35$ \\
\hline phase of $V \eta_{1}$ & $-2.6 \pm 2.5$ \\
\hline phase of $\mathrm{PV}_{1}$ & $0.28 \pm 1.5$ \\
\hline phase of $\mathrm{PV}_{8^{\prime}}$ & $-1.47 \pm 0.38$ \\
\hline phase of $P V_{10}$ & $-2.49 \pm 0.30$ \\
\hline phase of $P V_{\overline{10}}$ & $2.61 \pm 0.23$ \\
\hline phase of $\mathrm{PV}_{27}$ & $-2.86 \pm 0.52$ \\
\hline
\end{tabular}


Table 4.4: $\quad$ Modes with positive experimental values. Branching ratios from data and from the fit are given.

\begin{tabular}{|l|ll|ll|}
\hline Mode & Data BR & \multicolumn{2}{|l|}{ Fit BR } \\
\hline$D^{0} \rightarrow K^{-} \pi^{+}$ & 0.0401 & \pm 0.0014 & 0.0400 & \pm 0.0014 \\
$D^{0} \rightarrow K^{-} K^{+}$ & $0.00454 \pm 0.00029$ & 0.00453 & \pm 0.00030 \\
$D^{0} \rightarrow \bar{K}^{0} \pi^{0}$ & 0.0205 & \pm 0.0026 & 0.0208 & \pm 0.0022 \\
$D^{0} \rightarrow \bar{K}^{0} K^{0}$ & 0.0011 & \pm 0.0004 & 0.00103 & \pm 0.00043 \\
$D^{0} \rightarrow \pi^{-} \pi^{+}$ & $0.00159 \pm 0.00012$ & 0.00159 & \pm 0.00012 \\
$D^{0} \rightarrow \pi^{-} K^{+}$ & 0.00031 & \pm 0.00014 & 0.00031 & \pm 0.00018 \\
$D^{0} \rightarrow \pi^{0} \pi^{0}$ & $0.00088 \pm 0.00023$ & 0.00087 & \pm 0.00025 \\
$D^{0} \rightarrow \eta \bar{K}^{0}$ & 0.0068 & \pm 0.0011 & 0.0069 & \pm 0.0011 \\
$D^{0} \rightarrow \eta^{\prime} \bar{K}^{0}$ & 0.0166 & \pm 0.0029 & 0.0168 & \pm 0.0028 \\
$D^{0} \rightarrow K^{*-} \rho^{+}$ & 0.059 & \pm 0.024 & 0.063 & \pm 0.016 \\
$D^{0} \rightarrow \bar{K}^{* 0} \rho^{0}$ & 0.016 & \pm 0.004 & 0.0164 & \pm 0.0038 \\
$D^{0} \rightarrow \bar{K}^{* 0} K^{* 0}$ & 0.0029 & \pm 0.0015 & 0.0029 & \pm 0.0019 \\
$D^{0} \rightarrow \omega \bar{K}^{* 0}$ & 0.011 & \pm 0.005 & 0.0099 & \pm 0.0044 \\
$D^{0} \rightarrow \phi \rho^{0}$ & 0.0019 & \pm 0.0005 & 0.00192 & \pm 0.00045 \\
$D^{0} \rightarrow K^{-} \rho^{+}$ & 0.104 & \pm 0.013 & 0.102 & \pm 0.013 \\
$D^{0} \rightarrow K^{-} K^{*+}$ & 0.0034 & \pm 0.0008 & 0.00323 & \pm 0.00080 \\
$D^{0} \rightarrow \bar{K}^{0} \rho^{0}$ & 0.0110 & \pm 0.0018 & 0.0110 & \pm 0.0017 \\
$D^{0} \rightarrow K^{*-} \pi^{+}$ & 0.049 & \pm 0.006 & 0.0495 & \pm 0.0058 \\
$D^{0} \rightarrow K^{*-} K^{+}$ & 0.0018 & \pm 0.0010 & 0.00209 & \pm 0.00087 \\
$D^{0} \rightarrow \bar{K}^{* 0} \pi^{0}$ & 0.030 & \pm 0.004 & 0.0301 & \pm 0.0039 \\
$D^{0} \rightarrow \phi \bar{K}^{0}$ & 0.0083 & \pm 0.0012 & 0.0081 & \pm 0.0012 \\
$D^{0} \rightarrow \omega \bar{K}^{0}$ & 0.020 & \pm 0.004 & 0.0195 & \pm 0.0043 \\
$D^{0} \rightarrow \eta \bar{K}^{* 0}$ & 0.019 & \pm 0.005 & 0.0204 & \pm 0.0049 \\
\hline
\end{tabular}




\begin{tabular}{|l|ll|ll|}
\hline Mode & \multicolumn{2}{|l|}{ Data BR } & Fit BR \\
\hline$D^{+} \rightarrow \bar{K}^{0} \pi^{+}$ & 0.0274 & \pm 0.0029 & 0.0262 & \pm 0.0028 \\
$D^{+} \rightarrow \bar{K}^{0} K^{+}$ & 0.0078 & \pm 0.0017 & 0.0086 & \pm 0.0016 \\
$D^{+} \rightarrow \pi^{0} \pi^{+}$ & 0.0025 & \pm 0.0007 & 0.00257 & \pm 0.00067 \\
$D^{+} \rightarrow \eta \pi^{+}$ & 0.0075 & \pm 0.0025 & 0.0068 & \pm 0.0021 \\
$D^{+} \rightarrow \bar{K}^{* 0} \rho^{+}$ & 0.021 & \pm 0.014 & 0.0398 & \pm 0.0092 \\
$D^{+} \rightarrow \bar{K}^{* 0} K^{*+}$ & 0.026 & \pm 0.011 & 0.0090 & +0.0054 \\
$D^{+} \rightarrow \bar{K}^{0} \rho^{+}$ & 0.066 & \pm 0.025 & 0.071 & \pm 0.018 \\
$D^{+} \rightarrow \pi^{+} K^{* 0}$ & $0.00046 \pm 0.00015$ & 0.00046 & \pm 0.00014 \\
$D^{+} \rightarrow \bar{K}^{* 0} \pi^{+}$ & 0.022 & \pm 0.004 & 0.0217 & \pm 0.0041 \\
$D^{+} \rightarrow \bar{K}^{* 0} K^{+}$ & 0.0051 & \pm 0.0010 & 0.00463 & \pm 0.00097 \\
$D^{+} \rightarrow \phi \pi^{+}$ & 0.0067 & \pm 0.0008 & 0.00674 & \pm 0.00078 \\
$D^{+} \rightarrow \phi K^{+}$ & $0.00039 \pm 0.00020$ & 0.00039 & +0.00027 \\
\hline$D_{s} \rightarrow \bar{K}^{0} K^{+}$ & 0.035 & \pm 0.007 & 0.0319 & \pm 0.0059 \\
$D_{s} \rightarrow \eta \pi^{+}$ & 0.019 & \pm 0.004 & 0.0204 & \pm 0.0039 \\
$D_{s} \rightarrow \eta^{\prime} \pi^{+}$ & 0.047 & \pm 0.014 & 0.054 & \pm 0.012 \\
$D_{s} \rightarrow \bar{K}^{* 0} K^{*+}$ & 0.056 & \pm 0.021 & 0.055 & \pm 0.018 \\
$D_{s} \rightarrow \phi \rho^{+}$ & 0.065 & \pm 0.017 & 0.056 & \pm 0.014 \\
$D_{s} \rightarrow \bar{K}^{0} K^{*+}$ & 0.042 & \pm 0.010 & 0.043 & \pm 0.011 \\
$D_{s} \rightarrow \bar{K}^{* 0} K^{+}$ & 0.033 & \pm 0.005 & 0.0328 & \pm 0.0053 \\
$D_{s} \rightarrow \phi \pi^{+}$ & 0.035 & \pm 0.004 & 0.0349 & \pm 0.0040 \\
$D_{s} \rightarrow \eta \rho^{+}$ & 0.100 & \pm 0.022 & 0.100 & \pm 0.019 \\
\hline
\end{tabular}




\subsection{Predictions}

\subsubsection{Predictions from the Fit Parameters}

From the fit values of the parameters the branching ratios of decay modes were calculated. In Table 4.4 are presented the modes for which there exist experimental values. Our calculated branching ratios are consistent with the data, with the exception of $D^{+} \rightarrow \bar{K}^{* 0} K^{*+}$ and $D_{s} \rightarrow \eta^{\prime} \rho^{+}$. For the former the fit prefers a branching ratio that is three standard deviations below the reported experimental value. The latter was removed before the fit (see Section 4.4) because its experimental value was questioned. For this mode we predict a branching ratio of $\left(1.5_{-1.1}^{+1.9}\right) \%$, well below the reported experimental value [59]. Tables 4.5-4.7 contain modes for which there is no experimental information or for which there is an experimental limit. We have attempted to predict the branching ratio of each mode from the fit. However, in some cases the uncertainties are so large that we are able only to provide (90\% confidence level) limits on the branching ratios. Notice that in all cases in which there are experimental limits, our predicted branching ratio or predicted limit is in the allowed region. We are unable to say anything about the mode $D_{s} \rightarrow \rho^{0} \pi^{+}$, because the uncertainty on its prediction is greater than the experimental limit.

There are two modes, $D_{s} \rightarrow \pi^{+} \pi^{0}$ and $D_{s} \rightarrow \rho^{+} \rho^{0}$, which are forbidden in a model without isospin breaking. The modes that are kinematically forbidden are $D^{0} \rightarrow \eta^{\prime} \eta^{\prime}, \eta^{\prime} \phi$, and $\phi \phi$. The modes involving the linear combinations $L^{(1)}, L^{(2)}$, and $L^{(3)}$ are discussed below. Any other PP, PV, or VV mode not appearing in the tables is higher order in the weak coupling $G_{F}$.

\subsubsection{Unconstrained Linear Combinations}

There remain three linearly independent combinations of the reduced matrix elements that are not constrained by the data. The combination $L^{(1)}$ contributes only to modes of the type $D^{0} \rightarrow \eta \eta$. $L^{(2)}$ contributes to the types $D^{0} \rightarrow \eta \eta$ and 
Table 4.5: $\quad D^{0}$ modes with predicted branching ratios. Experimental limits are given when available. All limits are at $90 \%$ confidence.

\begin{tabular}{|c|c|c|c|}
\hline Mode & Data BR & Predicted BR & Predicted limit \\
\hline$D^{0} \rightarrow \pi^{0} K^{0}$ & & $0.00017_{-0.00008}^{+0.00011}$ & \\
\hline$D^{0} \rightarrow \phi \bar{K}^{* 0}$ & & $0.00108_{-0.00054}^{+0.00073}$ & \\
\hline$D^{0} \rightarrow \rho^{0} K^{* 0}$ & & $0.00038_{-0.00022}^{+0.00031}$ & \\
\hline$D^{0} \rightarrow \pi^{0} \rho^{0}$ & & $0.00014{ }_{-0.00011}^{+0.00018}$ & \\
\hline$D^{0} \rightarrow \pi^{-} \rho^{+}$ & & $0.093 \quad{ }_{-0.075}^{+0.133}$ & \\
\hline$D^{0} \rightarrow \rho^{-} \pi^{+}$ & . & $0.094 \quad{ }_{-0.076}^{+0.136}$ & \\
\hline$D^{0} \rightarrow \eta \pi^{0}$ & & $0.0060 \quad{ }_{-0.0050}^{+0.0092}$ & \\
\hline$D^{0} \rightarrow \phi \pi^{0}$ & & $0.024 \quad{ }_{-0.022}^{+0.049}$ & \\
\hline$D^{0} \rightarrow \eta \rho^{0}$ & & $0.025 \quad{ }_{-0.021}^{+0.041}$ & $<0.092$ \\
\hline$D^{0} \rightarrow K^{*-} K^{*+}$ & & $0.0024 \quad{ }_{-0.0021}^{+0.0041}$ & $<0.0092$ \\
\hline$D^{0} \rightarrow \rho^{0} K^{0}$ & & $0.0024 \quad{ }_{-0.0021}^{+0.0041}$ & $<0.0091$ \\
\hline$D^{0} \rightarrow \eta^{\prime} \overline{K^{* 0}}$ & $<0.0011$ & $0.00018{ }_{-0.00016}^{+0.00032}$ & $<0.00070$ \\
\hline$D^{0} \rightarrow \rho^{-} K^{*+}$ & & $0.00022{ }_{-0.00020}^{+0.00038}$ & $<0.00085$ \\
\hline$D^{0} \rightarrow \rho^{-} K^{+}$ & & $0.0020 \quad{ }_{-0.0018}^{+0.0035}$ & $<0.0078$ \\
\hline$D^{0} \rightarrow \pi^{-} K^{*+}$ & & $0.0019 \quad{ }_{-0.0018}^{+0.0037}$ & $<0.0080$ \\
\hline$D^{0} \rightarrow \pi^{0} K^{* 0}$ & & $0.0020 \quad{ }_{-0.0019}^{+0.0041}$ & $<0.0087$ \\
\hline$D^{0} \rightarrow \bar{K}^{0} K^{* 0}$ & $<0.0008$ & & $<0.00052$ \\
\hline$D^{0} \rightarrow \omega \pi^{0}$ & & & $<0.086$ \\
\hline$D^{0} \rightarrow \eta^{\prime} \rho^{0}$ & & & $<0.011$ \\
\hline$D^{0} \rightarrow \omega \rho^{0}$ & & & $<0.084$ \\
\hline$D^{0} \rightarrow \rho^{-} \rho^{+}$ & & & $<0.015$ \\
\hline$D^{0} \rightarrow \bar{K}^{* 0} K^{0}$ & $<0.0015$ & & $<0.00061$ \\
\hline$D^{0} \rightarrow \eta^{\prime} \pi^{0}$ & & & $<0.057$ \\
\hline$D^{0} \rightarrow \rho^{0} \rho^{0}$ & & & $<0.0065$ \\
\hline
\end{tabular}


Table 4.6: $\quad D^{+}$modes with predicted branching ratios. Experimental limits are given when available. All limits are at $90 \%$ confidence.

\begin{tabular}{|l|l|ll|l|}
\hline Mode & Data BR & Predicted BR & Predicted limit \\
\hline$D^{+} \rightarrow \rho^{0} \rho^{+}$ & & 0.0066 & \pm 0.0023 & \\
$D^{+} \rightarrow \eta K^{+}$ & & 0.0032 & ${ }_{-0.0020}^{+0.0030}$ & \\
$D^{+} \rightarrow \pi^{+} K^{0}$ & & 0.017 & ${ }_{-0.011}^{+0.018}$ & \\
$D^{+} \rightarrow \pi^{0} K^{+}$ & & 0.0086 & ${ }_{-0.0057}^{+0.0089}$ & \\
$D^{+} \rightarrow \pi^{0} \rho^{+}$ & & 0.0034 & ${ }_{-0.0023}^{+0.0036}$ & \\
$D^{+} \rightarrow \phi K^{*+}$ & & 0.00031 & ${ }_{-0.00022}^{+0.00035}$ & \\
$D^{+} \rightarrow \rho^{+} K^{* 0}$ & & 0.025 & ${ }_{-0.018}^{+0.031}$ & \\
$D^{+} \rightarrow \rho^{0} K^{*+}$ & & 0.0095 & ${ }_{-0.0071}^{+0.0118}$ & \\
$D^{+} \rightarrow \rho^{+} K^{0}$ & & 0.0087 & ${ }_{-0.0068}^{+0.0119}$ & \\
$D^{+} \rightarrow \omega K^{*+}$ & & 0.0022 & ${ }_{-0.0018}^{+0.0031}$ & \\
$D^{+} \rightarrow \omega \pi^{+}$ & $<0.007$ & 0.0024 & ${ }_{-0.0020}^{+0.0036}$ & \\
$D^{+} \rightarrow \pi^{0} K^{*+}$ & & 0.0103 & ${ }_{-0.0087}^{+0.0162}$ & \\
$D^{+} \rightarrow \omega \rho^{+}$ & & 0.0026 & ${ }_{-0.0023}^{+0.0049}$ & $<0.011$ \\
$D^{+} \rightarrow \bar{K}^{0} K^{*+}$ & & 0.0012 & ${ }_{-0.0011}^{+0.0026}$ & $<0.0054$ \\
$D^{+} \rightarrow \eta \rho^{+}$ & $<0.012$ & 0.0012 & ${ }_{-0.0011}^{+0.0022}$ & $<0.0048$ \\
$D^{+} \rightarrow \eta^{\prime} K^{+}$ & & 0.0016 & ${ }_{-0.0015}^{+0.0041}$ & $<0.0082$ \\
$D^{+} \rightarrow \rho^{0} K^{+}$ & & 0.0018 & ${ }_{-0.0017}^{+0.0042}$ & $<0.0086$ \\
$D^{+} \rightarrow \eta^{\prime} \pi^{+}$ & $<0.009$ & 0.00094 & ${ }_{-0.00092}^{+0.00237}$ & $<0.0048$ \\
$D^{+} \rightarrow \phi \rho^{+}$ & $<0.015$ & & & $<0.0074$ \\
$D^{+} \rightarrow \omega K^{+}$ & & & & $<0.0012$ \\
$D^{+} \rightarrow \eta^{\prime} \rho^{+}$ & $<0.015$ & & & $<0.00071$ \\
$D^{+} \rightarrow \rho^{0} \pi^{+}$ & $<0.0014$ & & & $<0.00091$ \\
$D^{+} \rightarrow \eta^{\prime} K^{*+}$ & & & & \\
$D^{+} \rightarrow \eta K^{*+}$ & & & & \\
\hline
\end{tabular}


Table 4.7: $\quad D_{s}$ modes with predicted branching ratios. Experimental limits are given when available. All limits are at $90 \%$ confidence. The measured branching ratio of the mode $D_{s} \rightarrow \eta^{\prime} \rho^{+}$is discussed in the text.

\begin{tabular}{|c|c|c|c|}
\hline Mode & Data BR & Predicted $B R$ & Predicted limit \\
\hline$D_{s} \rightarrow \pi^{0} K^{+}$ & & $0.0059{ }_{-0.0034}^{+0.0048}$ & \\
\hline$D_{s} \rightarrow \pi^{+} K^{* 0}$ & & $0.038 \quad{ }_{-0.028}^{+0.047}$ & \\
\hline$D_{s} \rightarrow \eta^{\prime} \rho^{+}$ & $0.120 \pm 0.030$ & $0.015 \quad{ }_{-0.011}^{+0.019}$ & \\
\hline$D_{s} \rightarrow \pi^{0} K^{*+}$ & & $0.077 \quad \begin{array}{ll}+0.096 \\
-0.058\end{array}$ & \\
\hline$D_{s} \rightarrow \rho^{0} K^{*+}$ & & $0.0126{ }_{-0.0096}^{+0.0164}$ & \\
\hline$D_{s} \rightarrow \rho^{+} K^{0}$ & & $0.031 \quad{ }_{-0.024}^{+0.043}$ & \\
\hline$D_{s} \rightarrow \rho^{0} K^{+}$ & & $0.049 \quad{ }_{-0.040}^{+0.071}$ & \\
\hline$D_{s} \rightarrow \omega \rho^{+}$ & & $0.012 \quad{ }_{-0.012}^{+0.030}$ & $<0.061$ \\
\hline$D_{s} \rightarrow \pi^{+} K^{0}$ & $<0.007$ & & $<0.0015$ \\
\hline$D_{s} \rightarrow K^{0} K^{*+}$ & & & $<0.00039$ \\
\hline$D_{s} \rightarrow K^{0} K^{+}$ & & & $<0.00046$ \\
\hline$D_{s} \rightarrow K^{* 0} K^{*+}$ & & & $<0.00057$ \\
\hline$D_{s} \rightarrow \rho^{+} K^{* 0}$ & & & $<0.0080$ \\
\hline$D_{s} \rightarrow K^{* 0} K^{+}$ & & & $<0.00025$ \\
\hline$D_{s} \rightarrow \omega \pi^{+}$ & $<0.017$ & & $<0.0090$ \\
\hline$D_{s} \rightarrow \pi^{0} \rho^{+}$ & & & $<0.064$ \\
\hline$D_{s} \rightarrow \rho^{0} \pi^{+}$ & $<0.0028$ & & not significant \\
\hline$D_{s} \rightarrow \pi^{+} \pi^{0}$ & & $\equiv 0$ & \\
\hline$D_{s} \rightarrow \rho^{+} \rho^{0}$ & & $\equiv 0$ & \\
\hline
\end{tabular}


$D^{\circ} \rightarrow \eta K^{0} . L^{(3)}$ contributes to these modes, and to modes of the type $D_{s} \rightarrow \eta K^{+}$. The first unconstrained linear combination $L^{(1)}$ contributes only to amplitudes involving $(S S)_{1}$. These amplitudes, it is worth noting, are due entirely to $S U(3)$ breaking. However, when we include the phases, we must make four estimates in order to obtain two predictions of modes of the type $D^{0} \rightarrow \eta \eta$. This would be an unproductive endeavor, and so we forego it.

In order to predict the modes of the types $D^{0} \rightarrow \eta K^{0}$ and $D_{s} \rightarrow \eta K^{+}$, we need two new inputs. In order to show the variability of the resulting predictions, we try three different sets of inputs. Scheme A is motivated by the recent CLEO measurement of the doubly-suppressed mode $D^{0} \rightarrow \pi^{-} K^{+}[60]$, in which this mode is found to have a branching ratio of about three times that of the corresponding unsuppressed mode, $D^{0} \rightarrow \pi^{+} K^{-}$. For this scheme, the two inputs are

$$
\begin{aligned}
& B\left(D^{0} \rightarrow \eta K^{0}\right)=3 \tan ^{4} \theta_{C} B\left(D^{0} \rightarrow \eta \bar{K}^{0}\right), \\
& B\left(D_{s} \rightarrow \eta K^{+}\right)=3 \tan ^{2} \theta_{C} B\left(D_{s} \rightarrow \eta \pi^{+}\right) .
\end{aligned}
$$

The linear combinations $L^{(2)}$ and $L^{(3)}$ are then constrained and the remaining branching ratios in the column for scheme $\mathrm{A}$ in Table 4.8 are found. The predictions for scheme $\mathrm{B}$ are based on the following estimates:

$$
\begin{aligned}
& B\left(D^{0} \rightarrow \eta K^{0}\right)=3 \tan ^{4} \theta_{C} B\left(D^{0} \rightarrow \eta \bar{K}^{0}\right), \\
& B\left(D_{s} \rightarrow \phi K^{+}\right)=3 \tan ^{2} \theta_{C} B\left(D_{s} \rightarrow \phi \pi^{+}\right) .
\end{aligned}
$$

A third scheme $(\mathrm{C})$ is considered also. It is based on these estimates:

$$
\begin{aligned}
& B\left(D^{0} \rightarrow \phi K^{0}\right)=3 \tan ^{4} \theta_{C} B\left(D^{0} \rightarrow \phi \bar{K}^{0}\right), \\
& B\left(D_{s} \rightarrow \phi K^{+}\right)=\frac{1}{3} \tan ^{2} \theta_{C} B\left(D_{s} \rightarrow \phi \pi^{+}\right) .
\end{aligned}
$$

The resulting predictions are again in Table 4.8. The spread in these values provides an indication of the expected ranges for these quantities.

One should note that arbitrary choices of the above modes may fail to give an acceptable fit, given the constraints from measured modes. For example, an 
apparently reasonable choice would have been

$$
\begin{aligned}
& B\left(D^{0} \rightarrow \eta K^{0}\right)=\tan ^{4} \theta_{C} B\left(D^{0} \rightarrow \eta \bar{K}^{0}\right), \\
& B\left(D_{s} \rightarrow \eta K^{+}\right)=\tan ^{2} \theta_{C} B\left(D_{s} \rightarrow \eta \pi^{+}\right) .
\end{aligned}
$$

A consistent fit cannot be obtained to implement this. The parameters $L^{(2)}$ and $L^{(3)}$ could not be given values to accommodate $B\left(D^{0} \rightarrow \eta K^{0}\right)<0.0052 \%$.

\subsubsection{Modes Involving Axial Vectors}

There are a few modes involving axial vectors that have been observed or for which there are experimental limits. However, those that involve $K(1270)$ and $K(1400)$ are mixtures with the $1^{+-}$octet, which we can call $B$ since it includes the $b_{1}(1235)$. Therefore, in order to include these modes in our framework, we require two new parameters, "PA" and "PB." In addition, we must also accommodate the mixing between $f_{1(1)}$ and $f_{1(8)}$ to become $f_{1}(1285)$ and $f_{1}(1510)$, as well as the new phases that are introduced. There are too few experimental observations of the PA and PB modes to make this endeavor fruitful. For that reason, they are not included here.

\subsection{Comments on Models}

It is clear from the data alone that significant $S U(3)$ breaking is necessary in any successful model of $D$ decays. For example, $B\left(D^{0} \rightarrow K^{+} K^{-}\right)=B\left(D^{0} \rightarrow \pi^{+} \pi^{-}\right)$in exact $S U(3)$, yet they are in reality quite different. Models based on exact $S U(3)$ $[51],[55],[61]$ (or even on nonet symmetry [62]) are thus not admitted by the data.

Models of $D$ decays based on heavy-quark effective theory (e.g., [48]) have as yet not developed to the point at which individual nonleptonic decays can be calculated. The question of whether HQET is applicable to the $c$ quark is still unsettled. The HQET is based on an expansion in the parameter

$$
\frac{\Lambda_{\mathrm{QCD}}}{m_{\mathrm{C}}} \simeq 0.2
$$


Table 4.8: Modes based on estimates. The only available experimental limit is shown. Values marked with * are inputs.

\begin{tabular}{|l|l|l|l|l|}
\hline Mode & Data BR & $\begin{array}{l}\text { Fit BR } \\
\text { (scheme A) }\end{array}$ & $\begin{array}{l}\text { Fit BR } \\
\text { (scheme B) }\end{array}$ & $\begin{array}{l}\text { Fit BR } \\
\text { (scheme C) }\end{array}$ \\
\hline$D^{0} \rightarrow \eta K^{0}$ & & $0.000054^{*}$ & $0.000054^{*}$ & 0.00035 \\
$D^{0} \rightarrow \eta^{\prime} K^{0}$ & & 0.00046 & 0.00046 & 0.00085 \\
$D^{0} \rightarrow \phi K^{* 0}$ & & 0.000019 & 0.000019 & 0.000016 \\
$D^{0} \rightarrow \omega K^{* 0}$ & & 0.00027 & 0.00027 & 0.0012 \\
$D^{0} \rightarrow \phi K^{0}$ & & 0.00054 & 0.00054 & $0.000066^{*}$ \\
$D^{0} \rightarrow \omega K^{0}$ & & 0.000096 & 0.000096 & 0.0014 \\
$D^{0} \rightarrow \eta K^{* 0}$ & & 0.00048 & 0.00048 & 0.00094 \\
$D^{0} \rightarrow \eta^{\prime} K^{* 0}$ & & 0.0000083 & 0.0000083 & 0.0000024 \\
\hline$D_{s} \rightarrow \eta K^{+}$ & & $0.0027^{*}$ & 0.00041 & 0.0031 \\
$D_{s} \rightarrow \eta^{\prime} K^{+}$ & & 0.017 & 0.052 & 0.015 \\
$D_{s} \rightarrow \phi K^{*+}$ & & 0.011 & 0.024 & 0.0095 \\
$D_{s} \rightarrow \omega K^{*+}$ & & 0.0057 & 0.028 & 0.0046 \\
$D_{s} \rightarrow \phi K^{+}$ & $<0.0025$ & 0.00051 & $0.0033^{*}$ & $0.00037^{*}$ \\
$D_{s} \rightarrow \omega K^{+}$ & & 0.0064 & 0.019 & 0.0055 \\
$D_{s} \rightarrow \eta K^{*+}$ & & 0.00083 & 0.00015 & 0.00094 \\
$D_{s} \rightarrow \eta^{\prime} K^{*+}$ & & 0.00090 & 0.0028 & 0.00077 \\
\hline
\end{tabular}


and assumes that it is small. Certainly this would be a good assumption in the case of the $b$ quark, but perhaps not so here. Until we are able to calculate branching fractions in HQET, we must reserve judgement on its applicability to the $D$ mesons.

Diagrammatical approaches to the problem of $D$ decays present us with a complementary method to the one adopted in this work. The lowest-order diagrams are in Figures 4.2-4.7. Diagrammatical methods assign to each diagram a parameter and attempt to fit them to the data. The parameters in the $S U(3)$ framework represent sums of diagrams in the diagrammatical approach. A very general diagrammatical calculation of branching fractions appears in [50]. Two shortcomings of their work lie in final-state interactions and in the inclusion of $S U(3)$ breaking. The phases of the final-state interactions are added to the model, and are external to its central theme, and therefore appear as an ad-hoc mechanism to force a fit. $S U(3)$ breaking is added to the calculation as an additive correction to the diagrams in which it is believed to be important. However, there is also hidden breaking in the addition of phases in the final-state interactions. The result is a model in which the size and source of $S U(3)$ breaking is not easily discerned. It is difficult to draw any conclusions from the application of such a model.

The factorization method is a special case of the diagrammatical approach. In it, amplitudes are assumed to factorize into color-singlet pieces; the octet factors are neglected. Also, certain diagrams are considered unimportant (i.e., the annihilation diagrams are thought to produce two quarks at high momentum, and therefore contribute instead to multiparticle final states). However, [49] find that these diagrams must be again included in order to accommodate the data, and that final-state transitions and intermediate resonances must also be added. The result is an eclectic model with little elegance. We are unable, because of the ad-hoc features, to comment on the reliability and predictability of this model.

A description of nonleptonic $D$ decays in a large- $N_{c}$ (number of colors) expansion [47] is an elegant one with few parameters. In it, the source of $S U(3)$-breaking is introduced my including nearby resonances. It is also a subset of the diagrammat- 
Figure 4.2: $\quad$ External spectator diagram in the decays of $D$ mesons.

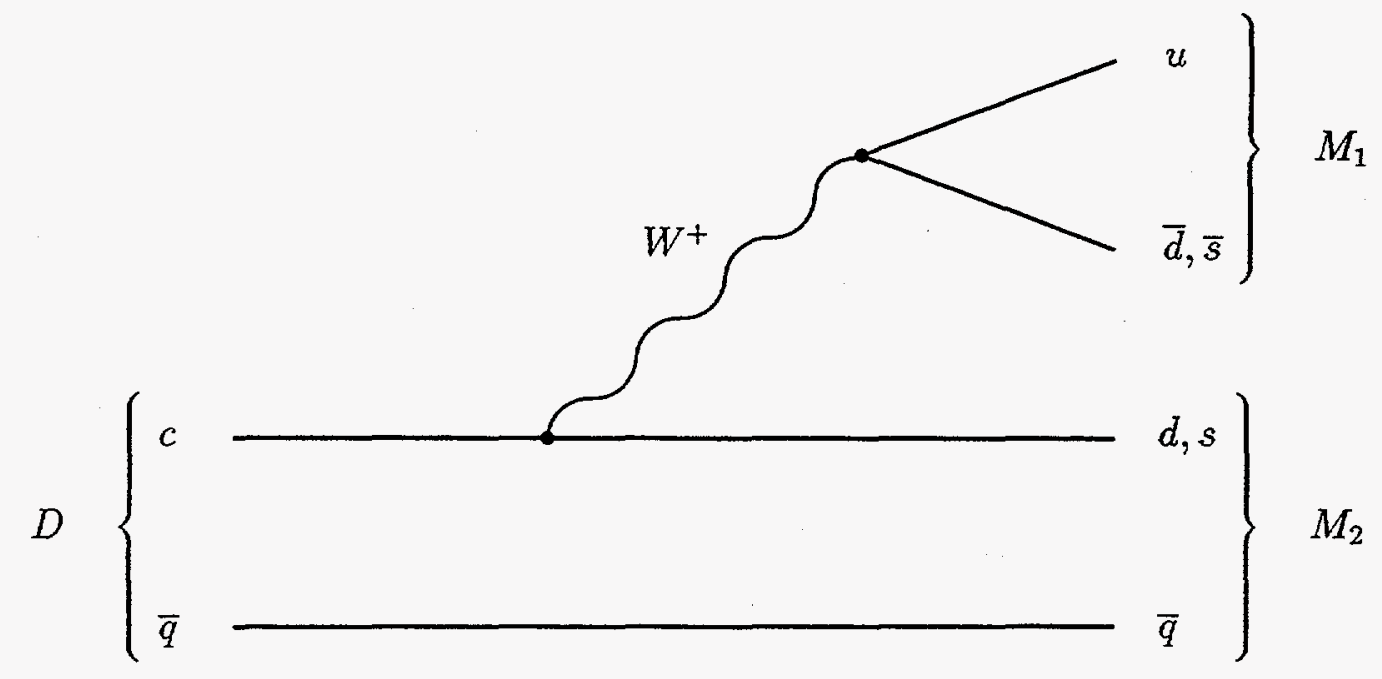

matical approach and neglects some diagrams based on their suppression by $1 / N_{c}$. One may argue that these diagrams are larger than thought and cite the fit of [50] as evidence of this. Nevertheless, [47] obtain excellent agreement with the data, with the exception of some modes involving $\eta$ and $\eta^{\prime}$. In this model, $S U(3)$ breaking is introduced only through the inclusion of resonances in one class of diagram. They obtain, in agreement with our work, large breaking.

\subsection{Conclusions}

There now exist enough data to constrain all but three combinations of the reduced matrix elements of the broken $S U(3)$ model of the decays of $D$ mesons with the two assumptions discussed in Section 4.2. We have used these data to do so. Using the experimental information on 57 modes we are able to predict branching ratios or upper limits for an additional 53 modes. Only two measured modes are not easily accommodated in the fit. The measurement of a six additional modes involving $\eta$, $\eta^{\prime}, \phi, \omega$ would enable another twenty modes to be predicted. 
Figure 4.3: Internal spectator diagram in the decays of $D$ mesons.

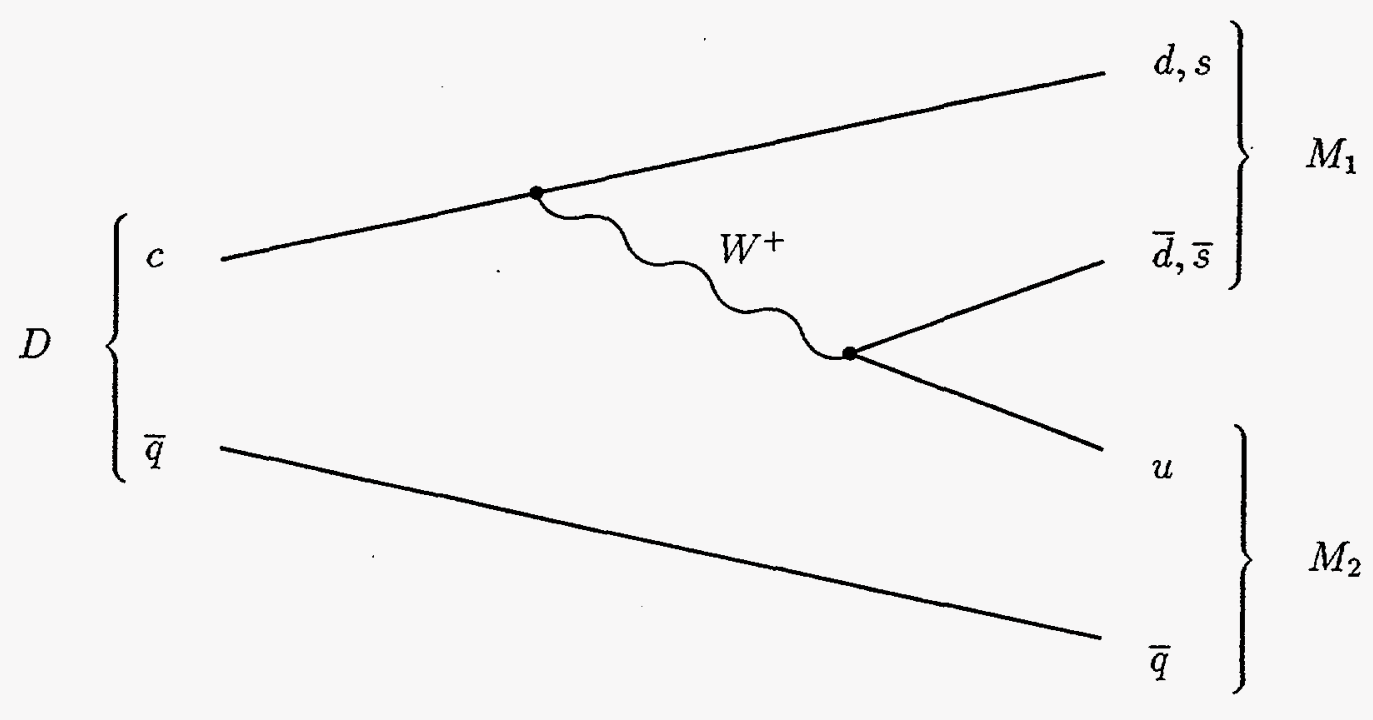

Figure 4.4: External annihilation diagram in the decays of $D$ mesons. Gluon fields are not shown.
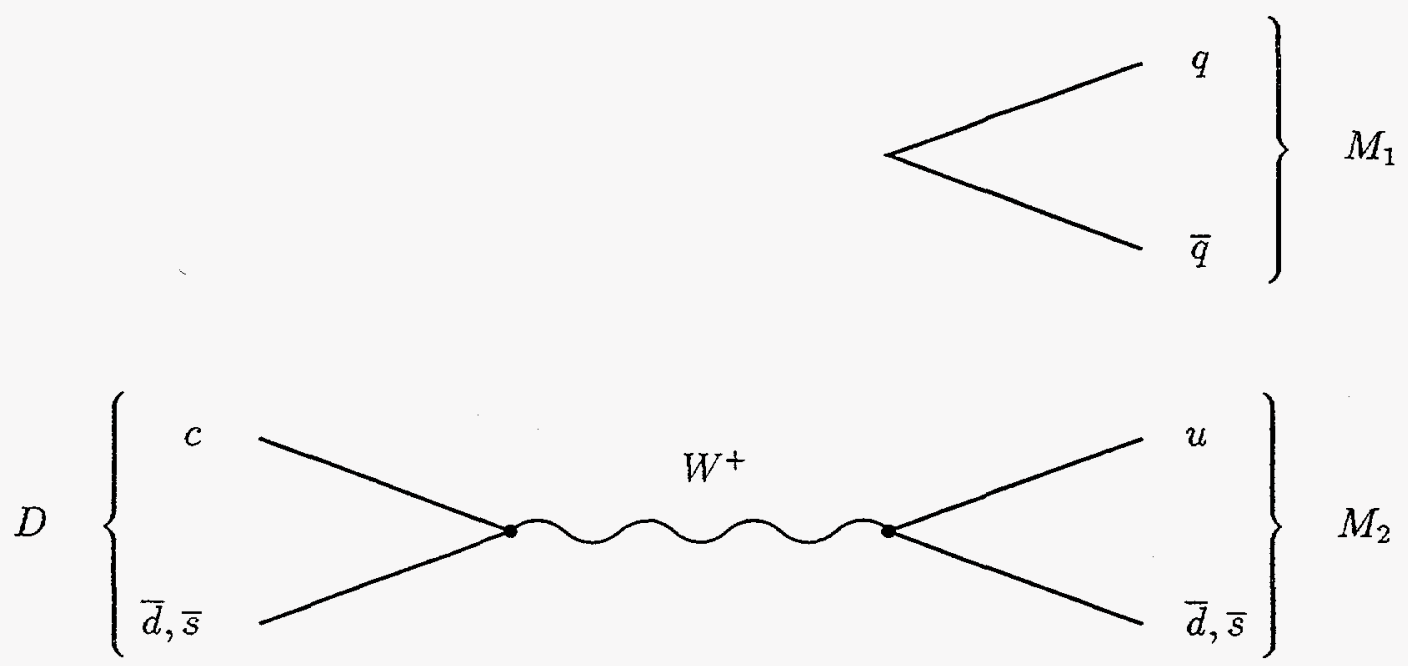
Figure 4.5: Internal annihilation diagram in the decays of $D$ mesons. Gluon fields are not shown.

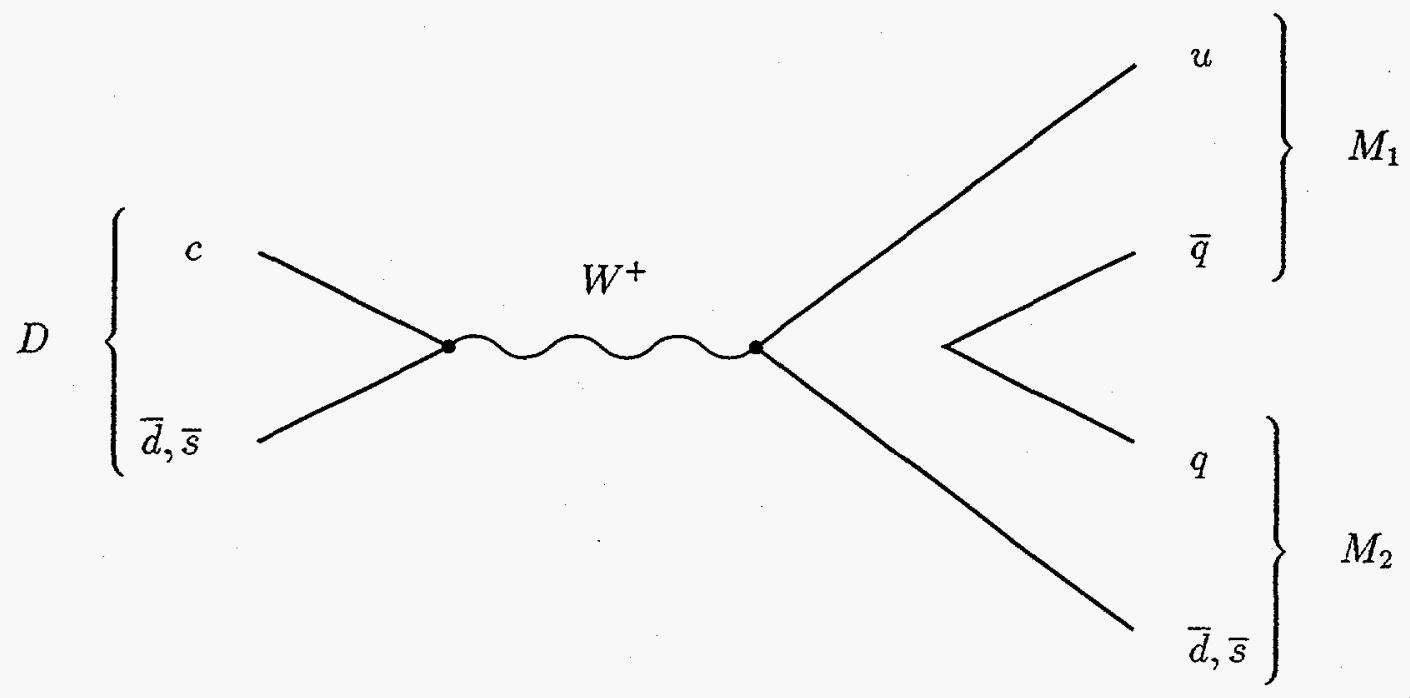

Figure 4.6: External exchange diagram in the decays of $D$ mesons. Gluon fields are not shown.
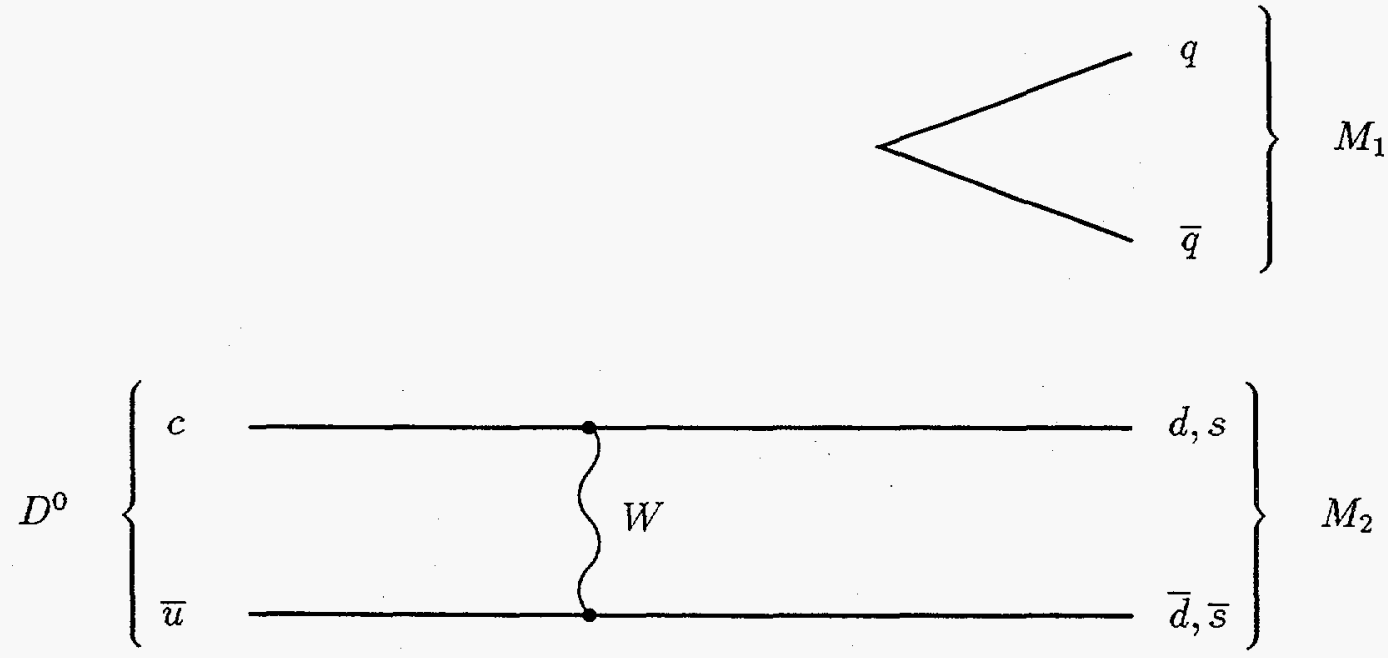
Figure 4.7: Internal exchange diagram in the decays of $D$ mesons. Gluon fields are not shown.

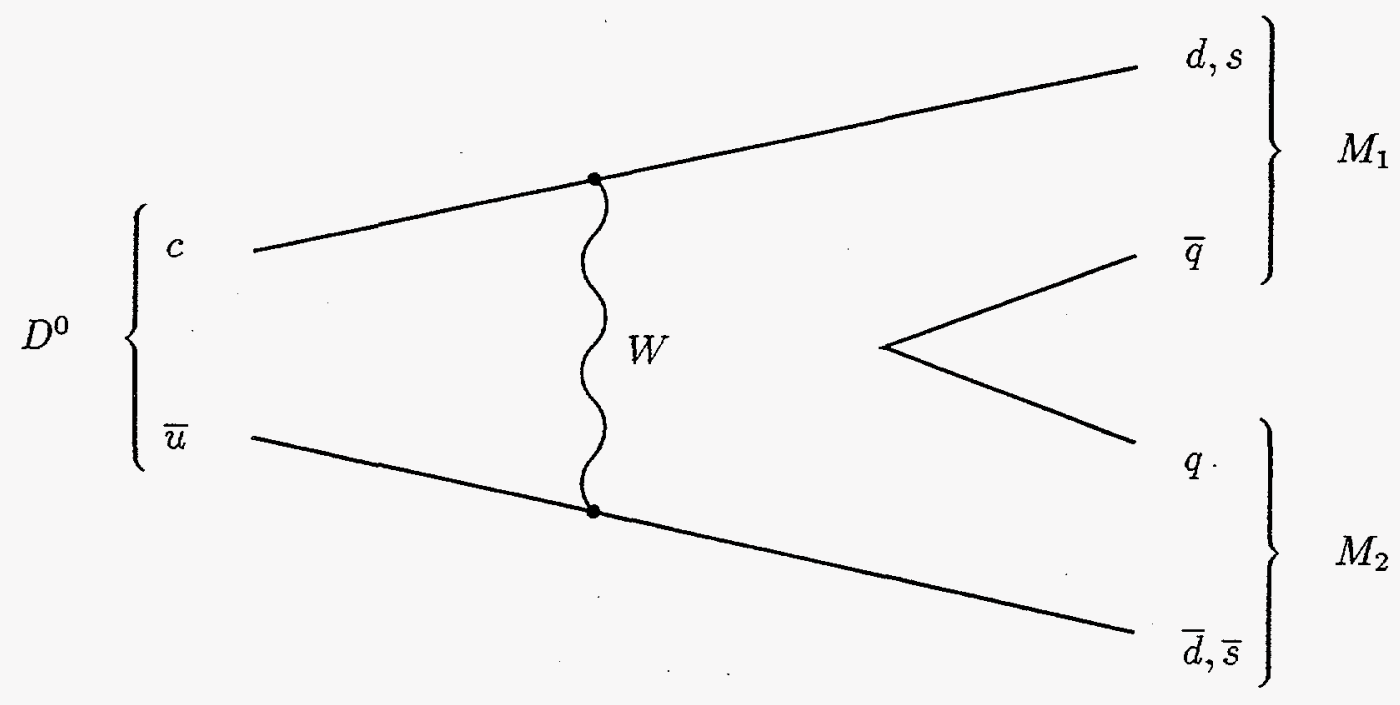




\section{$5 \quad D$-Meson Mixing in Broken Flavor $S U(3)$}

When two nearly degenerate states are related to one another by some interaction, then there is the possibility that they may mix. This is well known in the case of the the kaon system [63]. Here we will discuss the mixing of the neutral $D$ mesons. There is an interaction, second order in the weak coupling $G_{F}$, that introduces an off-diagonal entry in the mass matrix of the $D^{0}$ and $\bar{D}^{0}$ mesons. If we assume that $\mathrm{CP}$ is conserved, then we can write

$$
\mathcal{L}_{\text {mass }}=\left(D^{0} \bar{D}^{0}\right)\left(\begin{array}{cc}
M & \Delta m \\
\Delta m & M
\end{array}\right)\left(\begin{array}{c}
D^{0} \\
\bar{D}^{0}
\end{array}\right) .
$$

After the mass matrix is diagonalized, we find that the mass eigenstates are mixtures of the flavor eigenstates:

$$
\begin{aligned}
& D_{1}=\frac{1}{\sqrt{2}}\left(D^{0}+\bar{D}^{0}\right), \\
& D_{2}=\frac{1}{\sqrt{2}}\left(D^{0}-\bar{D}^{0}\right) .
\end{aligned}
$$

These are the CP odd and even eigenstates. Their masses are

$$
\begin{aligned}
& M_{1}=M+\Delta m, \\
& M_{2}=M-\Delta m .
\end{aligned}
$$

This $\Delta m$ conveniently parameterizes the mixing. Its estimates will be examined herein.

The chapter is organized as follows. First we will examine some past estimates of the short-and long-distance contributions. Then we will quote the results of an estimate based on the heavy-quark effective field theory. These estimates differ, 
and we will attempt to resolve the question by constructing an estimate of our own, based on the work of Chapter 4 .

\subsection{Short- and Long-Distance Contributions}

Depending on the scale of the momenta involved, either the short- or long-distance contributions are more important. In this section it is estimated that the longdistance contributions dominate.

The short-distance contributions to the mixing of the $D$ mesons are from the box diagrams in Figure 5.1. Various authors [64], [65], [66], [67] have estimated the size of these diagrams. Here we will review the estimate of Donoghue et al. [66]. The effective Lagrangian is

$$
\mathcal{L}=-\frac{G_{F}}{\sqrt{2}} \frac{\alpha}{8 \pi \sin ^{2} \theta_{\mathrm{w}}} \cos ^{2} \theta_{C} \sin ^{2} \theta_{C} x\left(\mathcal{O}+2 \mathcal{O}^{\prime}\right)
$$

where $x$ is

$$
x=\frac{\left(m_{s}^{2}-m_{d}^{2}\right)^{2}}{M_{W}^{2} m_{c}^{2}},
$$

and the operators $\mathcal{O}$ and $\mathcal{O}^{\prime}$ are

$$
\begin{aligned}
\mathcal{O} & =\bar{u} \gamma^{\mu}\left(1-\gamma_{5}\right) c \bar{u} \gamma_{\mu}\left(1-\gamma_{5}\right) c \\
\mathcal{O}^{\prime} & =\bar{u}\left(1+\gamma_{5}\right) c \bar{u}\left(1+\gamma_{5}\right) c
\end{aligned}
$$

The second of these operators arises from the large momentum of the external $c$ quarks that must be carried by the fermion propagators inside the boxes. The matrix elements of these operators can be estimated as

$$
\begin{aligned}
\left\langle\bar{D}^{0}|\mathcal{O}| D^{0}\right\rangle & =\frac{16}{3} \frac{m_{D}^{2} F_{D}^{2}}{2 m_{D}} B_{D} \\
\left\langle\bar{D}^{0}\left|\mathcal{O}^{\prime}\right| D^{0}\right\rangle & =-\frac{10}{3}\left(\frac{m_{D}}{m_{C}}\right)^{2} \frac{m_{D}^{2} F_{D}^{2}}{2 m_{D}} B_{D}^{\prime}
\end{aligned}
$$

These equations define the hadronic factors $B_{D}^{(\prime)}$. The $F_{D}$ is the pseudoscalar-decay constant:

$$
\left\langle 0\left|A_{\mu}\right| D^{0}(p)\right\rangle=i F_{D} p_{\mu}
$$


Figure 5.1: $\quad$ Box diagrams in $D$-meson mixing.
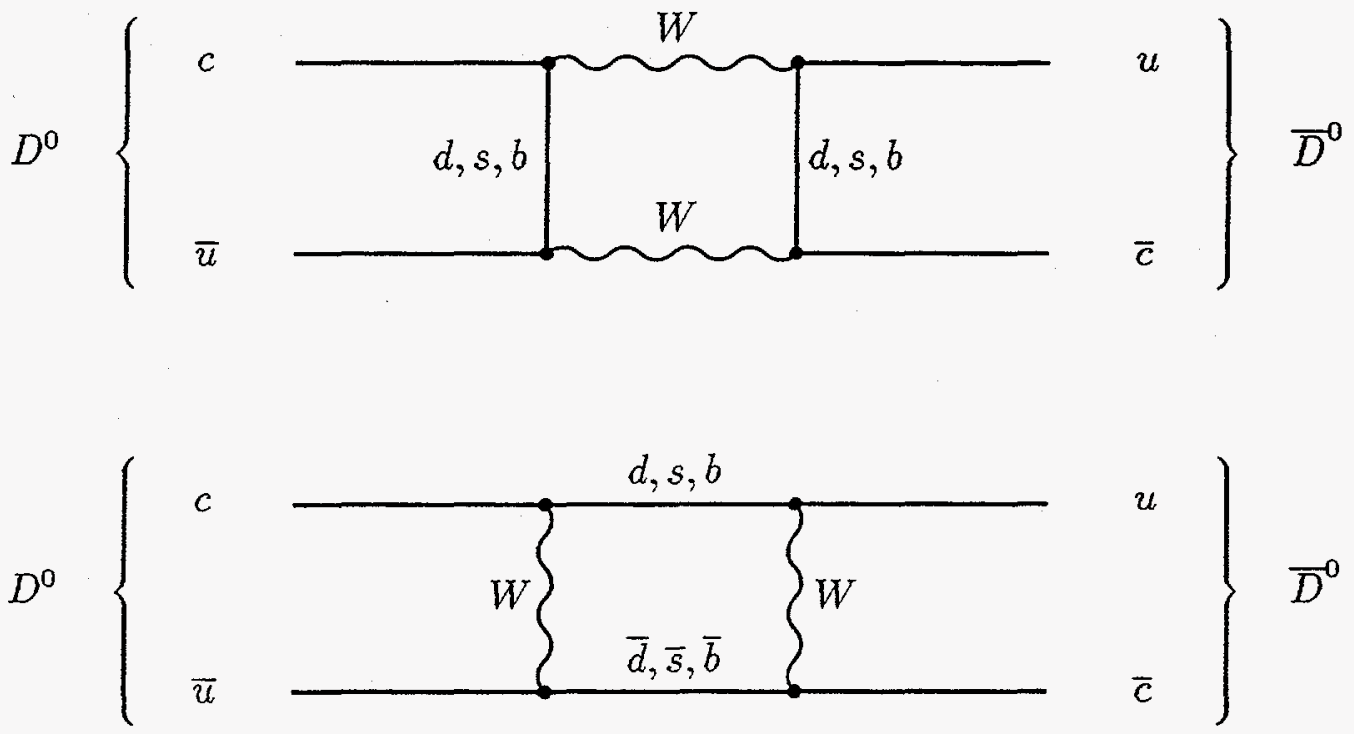

where $A_{\mu}$ is the axial current. The contribution of the box diagrams to the mass difference is

$$
\Delta m_{D}^{\text {box }}=-\frac{G_{F}}{\sqrt{2}} \frac{\alpha}{4 \pi \sin ^{2} \theta_{\mathrm{w}}} \cos ^{2} \theta_{C} \sin ^{2} \theta_{C} x m_{D} F_{D}^{2}\left(B_{D}-2 B_{D}^{\prime}\right) .
$$

If we assume that the hadronic factors $B \approx B^{\prime} \approx 1$, and take $F_{D} \approx 300 \mathrm{MeV}[10]$ and $m_{s} \approx 250 \mathrm{MeV}$, then the contribution of the box diagrams to $\Delta m_{D}$ is

$$
\Delta m_{D}^{\text {box }} \approx 4.8 \times 10^{-17} \mathrm{GeV}
$$

Notice that the $S U(3)$ breaking is manifest in the factor $x$ in the box calculation as $\left(m_{s}^{2}-m_{d}^{2}\right)^{2}$. The near cancellation in this factor is known as the GlashowIliopoulos-Maiani (GIM) mechanism. In the exact $S U(3)$ limit this factor would be zero, and hence the box contribution to $\Delta m_{D}$ would vanish.

The long-distance contributions to the mixing of $D$ mesons comes from the transition via intermediate hadronic states. Figure 5.2 shows the diagram for twobody intermediate state of mesons $M_{1}$ and $M_{2}$. An estimate of such a contribution 
can also be found in [66]. By considering the cutoff-dependent part of the diagram, one finds for the states involving $K^{ \pm}$and $\pi^{ \pm}$that

$$
\begin{aligned}
\Delta m_{D}^{K^{ \pm}, \pi^{ \pm}}= & \frac{1}{2 \pi} \ln \frac{m_{D}^{2}}{\mu^{2}}\left[\Gamma\left(D^{0} \rightarrow K^{+} K^{-}\right)+\Gamma\left(D^{0} \rightarrow \pi^{+} \pi^{-}\right)\right. \\
& \left.-2 \sqrt{\Gamma\left(D^{0} \rightarrow K^{+} \pi^{-}\right) \Gamma\left(D^{0} \rightarrow K^{-} \pi^{+}\right)}\right] .
\end{aligned}
$$

The scale $\mu$ is the cutoff above which the dispersive calculation is no longer valid. It is taken to be $\sim 1 \mathrm{GeV}$. By inserting the current values for the branching fractions $[10]$, we obtain

$$
\Delta m_{D}^{K^{ \pm}, \pi^{ \pm}}=(-0.75 \text { to } 0.29) \times 10^{-15} \mathrm{GeV},
$$

where the range of values is due to the uncertainty in the experimental value of $\Gamma\left(D^{0} \rightarrow K^{+} \pi^{-}\right)[60]$.

In this approach, the logarithm of Equation 5.11 treats the masses of all intermediate particles as small compared to $m_{D}$. This is not such a bad approximation, and a more careful treatment gives only a few percent corrections to individual modes. The use of the branching fractions in Equation 5.11 assumes that the couplings of the $D$ mesons to $\pi^{ \pm}$and $K^{ \pm}$are all relatively real. This we will correct in the last section of this chapter. Notice also that in the exact $S U(3)$ limit,

$$
\begin{aligned}
& \Gamma\left(D^{0} \rightarrow K^{+} K^{-}\right)=\Gamma\left(D^{0} \rightarrow \pi^{+} \pi^{-}\right) \\
& =\tan ^{2} \theta_{C} \Gamma\left(D^{0} \rightarrow K^{-} \pi^{+}\right)=\cot ^{2} \theta_{C} \Gamma\left(D^{0} \rightarrow K^{+} \pi^{-}\right),
\end{aligned}
$$

and hence the contribution to $\Delta m_{D}$ from Equation 5.11 vanishes. If, however, the only form of $S U(3)$ breaking were due to the differences in the quark masses, then the size of the contribution from Equation 5.11 would be only

$$
\Delta m_{D} \approx 10^{-17} \mathrm{GeV}
$$

It is the fact that $S U(3)$ breaking in the $D$ system is so large that gives such a large contribution to $\Delta m_{D}$ in Equation 5.12 . 
Figure 5.2: $\quad$ Two-body long-distance contribution to $D$-meson mixing.

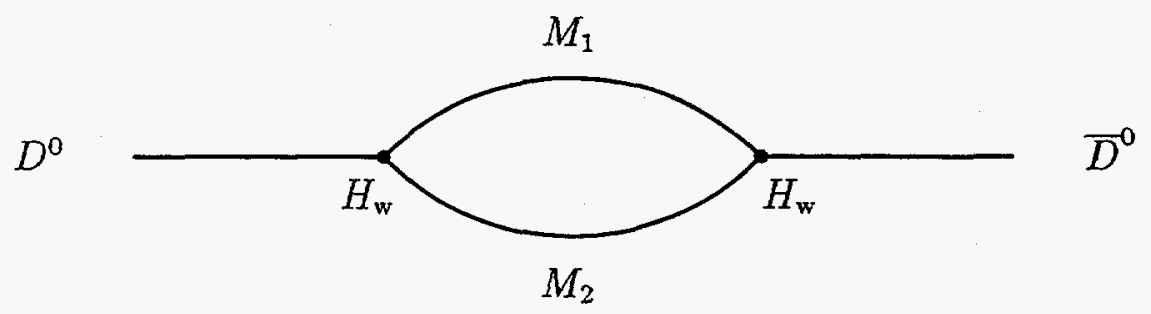

\subsection{Estimate in HQEFT}

An estimate of the mass difference in the heavy-quark effective field theory (HQEFT) was performed by Georgi et al.[68] [69]. They match operators in the HQEFT to the weak hamiltonian at the scale of the $W$ mass, and run them down to the $c$-quark scale. Then, after some naive estimates of hadronic matrix elements, they arrive at estimates for the contributions of 4-, 6-, and 8-quark operators:

$$
\begin{aligned}
& \Delta m_{D}^{4-\text {-quark }} \approx 1 \quad \times 10^{-17} \mathrm{GeV}, \\
& \Delta m_{D}^{6 \text {-quark }} \approx 2 \times 10^{-17} \mathrm{GeV}, \\
& \Delta m_{D}^{8 \text {-quark }} \approx 0.5 \times 10^{-17} \mathrm{GeV} .
\end{aligned}
$$

These estimates are all on the order of the size expected from the box diagrams, and are all much smaller than the simple two-body estimate of the long-distance contributions by Donoghue. We believe that this is due to the inadequate $S U(3)$ breaking in the treatment of the HQEFT. Here, only the quark-mass differences were used to break the flavor symmetry, and hence an estimate was obtained that is comparable to that in Equation 5.10. In order to resolve this problem, we have set out to make our own estimate of $\Delta m_{D}^{\mathrm{L}-\mathrm{D}}$. 
Table 5.1: $\quad$ Estimates of $\Delta m_{D}^{\mathrm{L}-\mathrm{D}}$. All values are in $10^{-15} \mathrm{GeV}$.

\begin{tabular}{c|ccc|c|} 
& PP & VV & PV & total \\
\hline no estimates & $7.3 \pm 3.9$ & $19.1 \pm 11.3$ & $-60.3 \pm 63.3$ & $-33.9 \pm 64.4$ \\
scheme A & $10.1 \pm 4.4$ & $25.5 \pm 11.9$ & $-56.5 \pm 63.9$ & $-20.9 \pm 65.1$ \\
scheme C & $4.6 \pm 4.5$ & $14.7 \pm 12.1$ & $-65.5 \pm 63.9$ & $-46.2 \pm 65.2$ \\
$K^{ \pm}$and $\pi^{ \pm}$ & $3.7 \pm 1.3$ & & & \\
\hline
\end{tabular}

\subsection{Mixing Estimate in Broken $S U(3)$}

We begin with the approach of Donoghue, with two improvements. First, the widths in Equation 5.11 are replaced by the complex vertices of Figure 5.2. Second, we include all pseudoscalar-pseudoscalar (PP), pseudoscalar-vector (PV), and vectorvector (VV) intermediate states that are considered in Chapter 4. Equation 5.11 is then replaced by

$$
\Delta m_{D}^{\mathrm{L}-\mathrm{D}}=\frac{1}{2 \pi} \ln \frac{m_{D}^{2}}{\mu^{2}} \times N \sum_{I} \mathcal{A}\left(D^{0} \rightarrow I\right) \mathcal{A}^{*}\left(D^{0} \rightarrow \bar{I}\right) .
$$

The sum is over all PP, PV, VV intermediate states and $N$ is a normalization factor determined by

$$
\Gamma\left(D^{0} \rightarrow K^{-} \pi^{+}\right)=N\left|\mathcal{A}\left(D^{0} \rightarrow K^{-} \pi^{+}\right)\right|^{2}
$$

The complex amplitudes are extracted from the fit of Section 4.4. The sum of Equation 5.16 is calculated for three cases. The first does not include the modes $D^{0} \rightarrow \eta^{(t)} K^{(*) 0}, \phi K^{(*) 0}, \omega K^{(*) 0}$. These are the modes for which we have an unconstrained parameter in the fit. The other two cases use the results of schemes $A$ and $\mathrm{C}$ of Section 4.5 .2 to estimate these modes. The estimates of $\Delta m_{D}^{\mathrm{L}-\mathrm{D}}$ are presented in Table 5.1 . 


\subsection{Conclusions}

We can see that the treatment of $D$-meson mixing by Donoghue et al. underestimates the uncertainties involved in the process. On the other hand, the treatment in HQEFT of Georgi et al. underestimates the flavor $S U(3)$ breaking that is involved. Therefore we have constructed our own estimate of the long-distance contributions to $D^{0}-\bar{D}^{0}$ mixing. We find that, in the absence of unlikely cancellations, the longdistance contributions are much larger than the short-distance contributions and are dominated by the PV intermediate states. However, the resulting uncertainties are large. The standard-physics contributions to $D$-meson mixing then can be as large as

$$
\Delta m_{D}^{\mathrm{L}-\mathrm{D}}=6 \times 10^{-14} \mathrm{GeV} .
$$

Therefore this process is less likely to be useful as a probe of new physics than previously thought [70]. 


\section{References}

[1] I. Hinchliffe and T. Kaeding, Phys. Rev. D47 (1993) 279.

[2] L. Lamport, LATEX: A Document Preparation System, Menlo Park, CA: Addison-Wesley, 1986.

[3] G. Altarelli, Les Houches Summer School: Particles in the Nineties, Les Houches, France, 1991.

[4] P. Langacker, Phys. Rep. 72 (1981) 185.

[5] U. Amaldi, W. de Boer, and H. Fürstenau, Phys. Lett. B260 (1991) 447.

[6] G. Kane, Modern Elementary Particle Physics, New York: Addison-Wesley, 1987.

[7] F. Mandl and G. Shaw, Quantum Field Theory, New York: John Wiley \& Sons, 1984.

[8] T.-P. Cheng and L.-F. Li, Gauge Theory of Elementary Particle Physics, Oxford: Oxford University Press, 1984.

[9] P. W. Higgs, Phys. Rev. 145 (1966) 1156.

[10] Particle Data Group, Review of Particle Properties, Phys. Rev. D50 (1994) 1173.

[11] H. Georgi and S. L. Glashow, Phys. Rev. Lett. 32 (1974) 438.

[12] IMB Collaboration, R. Becker-Szendy et al., Phys. Rev. D42 (1990) 2974.

[13] Wess and Bagger, Supersymmetry and Supergravity, second edition, Princeton, NJ: Princeton University Press, 1992.

[14] P. G. O. Freund, Introduction to Supersymmetry, New York: Cambridge University Press, 1986. 
[15] G. G. Ross, Supersymmetry: A Decade of Development, (ed. P. C. West) Boston: Hilger, 1986.

[16] I. Hinchliffe, Ann. Rev. Nucl. Part. Sci. 36 (1986) 505.

[17] R. Peccei and H. R. Quinn, Phys. Rev. D16 (1977) 1791.

[18] CDF Collaboration, F. Abe et al., Phys. Rev. Lett. 69 (1992) 3439.

[19] Fréjus Collaboration, Ch. Berger et al., Phys. Lett. B240 (1990) 237.

[20] C. B. Dover, A. Gal, and J. M. Richard, Phys. Rev. D27 (1983) 1090 and Phys. Rev. C31 (1985) 1423.

[21] R. Barbieri and A. Masiero, Nucl. Phys. B267 (1986) 679.

[22] F. Zwirner, Phys. Lett. 132B (1983) 103.

[23] J. L. Goity and M. Sher, Phys. Lett. B346 (1995) 69.

[24] SINDRUM Collaboration, U. Bellgardt et al., Nucl. Phys. B299 (1988) 1.

[25] Crystal Box Collaboration, R. D. Bolton et al., Phys. Rev. D38 (1988) 2077.

[26] B. Borodowsky et al., Phys. Rev. Lett. 68 (1992) 274.

[27] A. Antaramian, L. J. Hall, and A. Rašin, Phys. Rev. Lett. 69 (1992) 1871.

[28] L. E. Ibáñez and G. G. Ross, Phys. Lett. 260B (1991) 291, Nucl. Phys. B368 (1992) 3.

[29] P. L. White, Nucl. Phys. B403 (1993) 141.

[30] L. Krauss and F. Wilczek, Phys. Rev. Lett. 62 (1989) 1221.

[31] J. J. de Swart, Rev. Mod. Phys. 35 (1963) 916, Rev. Mod. Phys. 37 (1965) 326.

[32] P. McNamee and F. Chilton, Rev. Mod. Phys. 36 (1964) 1005. 
[33] V. Rabl et al., J. Math. Phys. 16 (1975) 2494.

[34] K. T. Hecht, Nucl. Phys. 62 (1965) 1.

[35] M. A. Rashid, Nuovo Cim. 26 (1962) 118.

[36] Y. Akiyama and J. P. Draayer, Comp. Phys. Comm. 5 (1973) 405.

[37] H. T. Williams, Symmetry Properties of $S U(3)$ Vector Coupling Coefficients, WLUPY-9-93.

[38] H. T. Williams and C. J. Wynne, Comp. in Phys. 8 (1994) 355.

[39] P. A. Carruthers, Introduction to Unitary Symmetry, New York: John Wiley \& Sons, 1966.

[40] H. Georgi, Lie Algebras in Particle Physics, London: Benjamin/Cummings, 1982.

[41] G. E. Baird and L. C. Biedenharn, J. Math. Phys. 4 (1963) 436, 4 (1963) 1449,5 (1964) 1723,5 (1964) 1730.

[42] E. U. Condon and G. H. Shortley, The Theory of Atomic Spectra, London: Cambridge University Press, 1957.

[43] R. Slansky, Phys. Rep. 79 (1981) 1.

[44] T. A. Kaeding, Comp. Phys. Comm. 85 (1995) 82.

[45] T. A. Kaeding, Tables of $S U(3)$ Isoscalar Factors, LBL-36659, UCB-PTH$95 / 01$.

[46] A. J. Buras et al., Nucl. Phys. B268 (1986) 16.

[47] D. Lee, Phys. Lett. B275 (1992) 469.

[48] I. I. Bigi, CERN-TH.7050/93, presented at Advanced Study Conference on Heavy Flavors, Pavia, Italy, 1993. 
[49] M. Bauer et al., Phys. Lett. 152B (1985) 380, Zeit. fur Physik C34 (1987) 103.

[50] L.-L. Chau et al., Phys. Rev. D36 (1987) 137, Phys. Lett. B222 (1989) 285, Phys. Rev. D42 (1990) 1837, Zeit. fur Physik C53 (1992) 413, Phys. Lett. B280 (1992) 281.

[51] C. Quigg, Zeit. fur Physik C4 (1980) 55.

[52] M. J. Savage, Phys. Lett. B257 (1991) 414, B259 (1991) 135.

[53] I. Hinchliffe and T. A. Kaeding, Nonleptonic Two-Body Decays of $D$ Mesons in Broken $S U(3)$, LBL-35892.

[54] Crystal Barrel Collaboration, C. Amsler et al., Phys. Lett. B294 (1992) 451.

[55] M. B. Einhorn et al., Phys. Rev. D12 (1975) 2015.

[56] F. J. Gilman and M. B. Wise, Phys. Rev. D20 (1979) 2392.

[57] E791 Collaboration, M. V. Purohit et al., XXVII International Conference on High Energy Physics, Glasgow, Scotland, 1994 (ed. P. J. Bussey and I. G. Knowles) Bristol: Institute of Physics Publishing.

[58] E691 Collaboration, J. C. Anjos et al., Phys. Rev. D46 (1992) 1941.

[59] CLEO Collaboration, P. Avery et al., Phys. Rev. Lett. 68 (1992) 1279.

[60] CLEO Collaboration, D. Cinabro et al., Phys. Rev. Lett. 72 (1994) 1406.

[61] R. L. Kingsley et al., Phys. Rev. D11 (1975) 1919.

[62] S. P. Rosen, Phys. Lett. B218 (1989) 353.

[63] For example, F. J. Gilman and M. B. Wise, Phys. Lett. 93B (1980) 129.

[64] A. J. Buras et al., Nucl. Phys. B238 (1984) 529. 
[65] J. S. Hagelin, Phys. Rev. D20 (1979) 2893.

[66] J. F. Donoghue et al., Phys. Rev. D33 (1986) 179.

[67] L. Wolfenstein, Phys. Lett. 164B (1985) 170.

[68] H. Georgi, Phys. Lett. B297 (1992) 353.

[69] T. Ohl et al., Nucl. Phys. B403 (1993) 605.

[70] J. L. Hewett, SLAC-PUB-6674, presented at the 1994 Meeting of the American Physical Society, Division of Particles and Fields, Albuquerque, 1994. 